\title{
Total Syntheses of Marrubiin and Related Labdane Diterpene Lactones
}

\author{
Yukari Sakagami, Naoki Kondo, Yuki Sawayama, Hiroyuki Yamakoshi $®$ and \\ Seiichi Nakamura *(i)
}

Graduate School of Pharmaceutical Sciences, Nagoya City University, 3-1 Tanabe-dori, Mizuho-ku, Nagoya 467-8603, Japan; yukarinnuu@gmail.com (Y.S.); c202709@ed.nagoya-cu.ac.jp (N.K.); ys020205@gmail.com (Y.S.); yamakoshi@phar.nagoya-cu.ac.jp (H.Y.)

* Correspondence: nakamura@phar.nagoya-cu.ac.jp; Tel.: +81-52-836-3439

Academic Editor: Marc C. Kimber

Received: 6 March 2020; Accepted: 30 March 2020; Published: 1 April 2020

\begin{abstract}
Total syntheses of the labdane diterpene lactones marrubiin, marrulibacetal, desertine, marrulibacetal A, marrubasch F, cyllenine C, marrulanic acid, and marrulactone are described. The trans-decalin moiety of these molecules was constructed in a stereoselective manner by a Pauson-Khand reaction, and the resultant cyclopentenone was oxidatively cleaved for formation of the lactone ring. Elongation of the side chain at C9 was achieved by an epoxide-opening reaction with a variety of nucleophiles, and the functional group manipulations completed the syntheses of these natural products. Stereochemistries of desertine could be established by the transformations.
\end{abstract}

Keywords: marrubiin; total synthesis; labdane; terpenoid; chiral building block; Pauson-Khand reaction

\section{Introduction}

Flowering plants of the genus Marrubium (Lamiaceae) are distributed in the Mediterranean and temperate regions of the Eurasian zone, and most of the plants are used in folk medicine [1]. The therapeutic properties of these herbs, including anti-inflammatory, hypoglycemic, analgesic, antispasmodic, vasorelaxant, and anti-diabetic effects, are attributed in part to marrubiin (1), which was first isolated in 1842 from Marrubium vulgare and is a prominent member of the labdane diterpene lactones [2] (Figure 1). This furanoid natural product, formed from premarrubiin (2), was also reported to inhibit KCl-induced contraction of the rat aorta in a concentration-dependent manner [3]. The important pharmacological action of this family of mints prompted phytochemical analysis, leading to the isolation and characterization of a number of labdane diterpene lactones [4]: peregrinine (3) [5], marrubinones A (4) and B (polyodonine, 5) [6,7], velutines A (6), B (7) and C (8) [8], marrulibanoside (9) [9], marrulanic acid (10) [10], cyllenines A (11) and C (12) [11], marrulibacetal (13), marrulactone (14) [12], marrusidins A (15) and B (16) [13], marrulibacetal A (17), desertine (18) [14], and marrubasch F (19) [15] have been reported to date [16]. These natural products are biosynthesized from $(E, E, E)$-geranylgeranyl diphosphate (GGPP) through peregrinol diphosphate synthase (CPS1)-catalyzed bicyclization, followed by 9,13-epoxylabd-14-ene synthase (ELS)-catalyzed formation of tetrahydropyran and regiospecific oxygenations with P450s [17,18]. Despite their remarkable biological activities, only a total synthesis of marrubiin (1) in a racemic form [19] and semi-syntheses of premarrubiin (2), marrulibanoside (9), marrubasch F (19) and (13R)-9 $\alpha, 13 \alpha$-epoxylabda-6 $\beta(19), 16$ (15)-diol dilactone from marrubiin (1) have been reported by Mangoni and co-workers [20].

Recently, we have developed a method for stereocontrolled preparation of enyne 20 (Figure 2, TMS = trimethylsilyl) by exploiting a ring-contractive coupling between an $\alpha$-bromo- $\delta$-valerolactone and a secondary alcohol, and subsequent Ireland-Claisen rearrangement [21,22]. Since compound 20 
embodies the C8-C10 stereotriad of C9-oxygenated labdane diterpenoids, compound 20 can serve as a useful chiral building block for the synthesis of pharmacologically interesting, marrubiin-related natural products. In this article, we describe the total syntheses of members of the marrubiin family including marrubiin (1), marrulanic acid (10), cyllenine C (12), marrulibacetal (13), marrulactone (14), marrulibacetal A (17), desertine (18), and marrubasch F (19) [23].

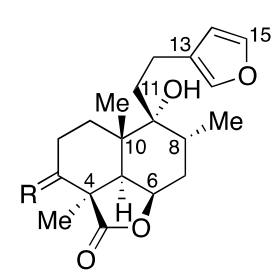

$\mathrm{R}=\mathrm{H}_{2}:$ marrubiin (1)

$\mathrm{R}=\mathrm{O}$ : peregrinine $(3)$

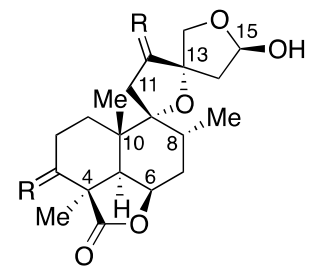

$\begin{array}{ll}R=O \text { : velutine } B(7) & R=O \text { : velutine } C(8) \\ R=H_{2}: \text { cyllenine } A(11) & R=H_{2}: \text { marrubasch }\end{array}$

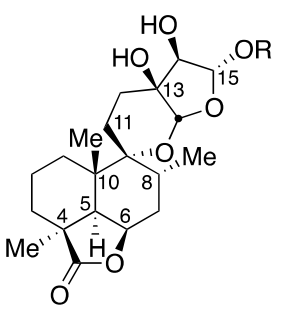

$\mathrm{R}=$ Et: marrulibacetal (13) $\mathrm{R}=\mathrm{Me}$ : marrulibacetal $\mathrm{A}(17)$

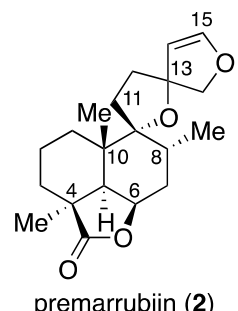

premarrubiin (2)

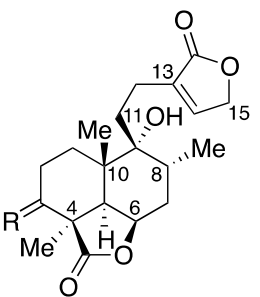

$\mathrm{R}=\mathrm{H}_{2}:$ marrubasch $\mathrm{F}(\mathbf{1 9})$

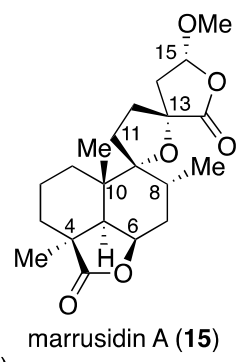

Figure 1. Structures of marrubiin and related natural products.

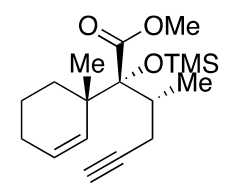

20

Figure 2. Structure of building block 20.

\section{Results and Discussion}

\subsection{Retrosynthetic Analysis}

Our retrosynthetic analysis of marrubiin-related natural products is depicted in Scheme 1 . Since the difference between the target molecules lies in the variation in the side chain at C9, we planned to install the full C9 side chain late in the synthesis by a nucleophilic ring-opening reaction of advanced epoxide intermediate 22, which could be derived from ester $\mathbf{2 3}$ through a chemoselective reduction of the ester functionality in the presence of the lactone moiety. While the methyl group at C4 would be introduced from the less-hindered diastereoface by an enolate alkylation, C5-C6 bond formation, carboxylation at $\mathrm{C} 4$, and oxidative cleavage of the $\mathrm{C}-\mathrm{C}$ bond at $\mathrm{C} 6$ were required for the conversion of enyne 20 to lactone 23. In light of these requirements, we chose to use the intramolecular Pauson-Khand reaction (PKR) $[24,25]$ to fashion the six-membered ring and introduce the carbonyl group at C4. Although 
some concern arose over the formation of cis-fused isomer cis-24 from enyne $\mathbf{2 0}$ considering that $\left[\mathrm{RhCl}(\mathrm{CO})_{2}\right]_{2}$-catalyzed PKRs of substituted 5-(pent-4-ynyl)cyclohexa-1,3-dienes are precedented to afford cis-decalin derivatives stereoselectively [26], we expected that the stereocenter at C5 would be epimerized after oxidative cleavage of the double bond at C6.

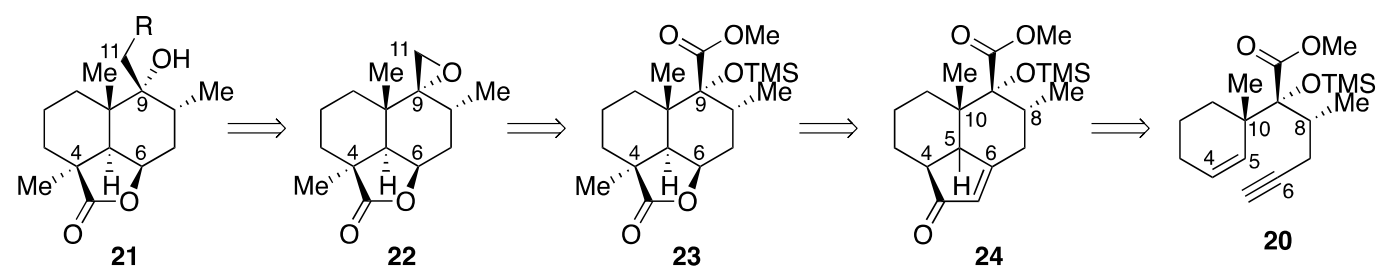

Scheme 1. Retrosynthetic analysis.

\subsection{Synthesis of Advanced Intermediate $\mathbf{2 2}$}

The synthesis of advanced intermediate $\mathbf{2 2}$ commenced with the key PKR of enyne $\mathbf{2 0}$ (Scheme 2). Initial attempts to cyclize dicobalt complex 25, prepared by treatment of enyne 20 with $\mathrm{Co}_{2}(\mathrm{CO})_{8}$ in $\mathrm{CH}_{2} \mathrm{Cl}_{2}$ (97\% yield), upon heating in refluxing acetonitrile failed to produce any of the PKR products, leading to decomplexation. After considerable experimentation, we found that the desired tricyclic compound 24 could be produced with the aid of a promoter, with $\mathrm{CyNH}_{2}(\mathrm{Cy}=$ cyclohexyl) [27] being optimal for this purpose. Gratifyingly, the product, obtained in $\left(\mathrm{CH}_{2} \mathrm{Cl}\right)_{2}$ at a substrate concentration of $10 \mathrm{mM}$ under optimized conditions in 97\% yield, proved to be the desired stereoisomer trans-24 as confirmed by the absence of a cross-peak between $\mathrm{C}_{10}-\mathrm{CH}_{3}$ and $\mathrm{C} 5-\mathrm{H}$ in the nuclear Overhauser effect spectroscopy (NOESY) spectrum. It has been suggested on the basis of quantum mechanical studies that the stereochemistry of PKR is determined by the irreversible olefin insertion step [28]. Since the chair-chairlike transition state (TS) A suffers from steric repulsion between $\mathrm{C} 8-\mathrm{CH}_{3}$ and $\mathrm{C} 1-\mathrm{H}_{\mathrm{ax}}$, the reaction proceeded through chair-boatlike TS B, leading to the exclusive formation of trans-decalin trans-24.

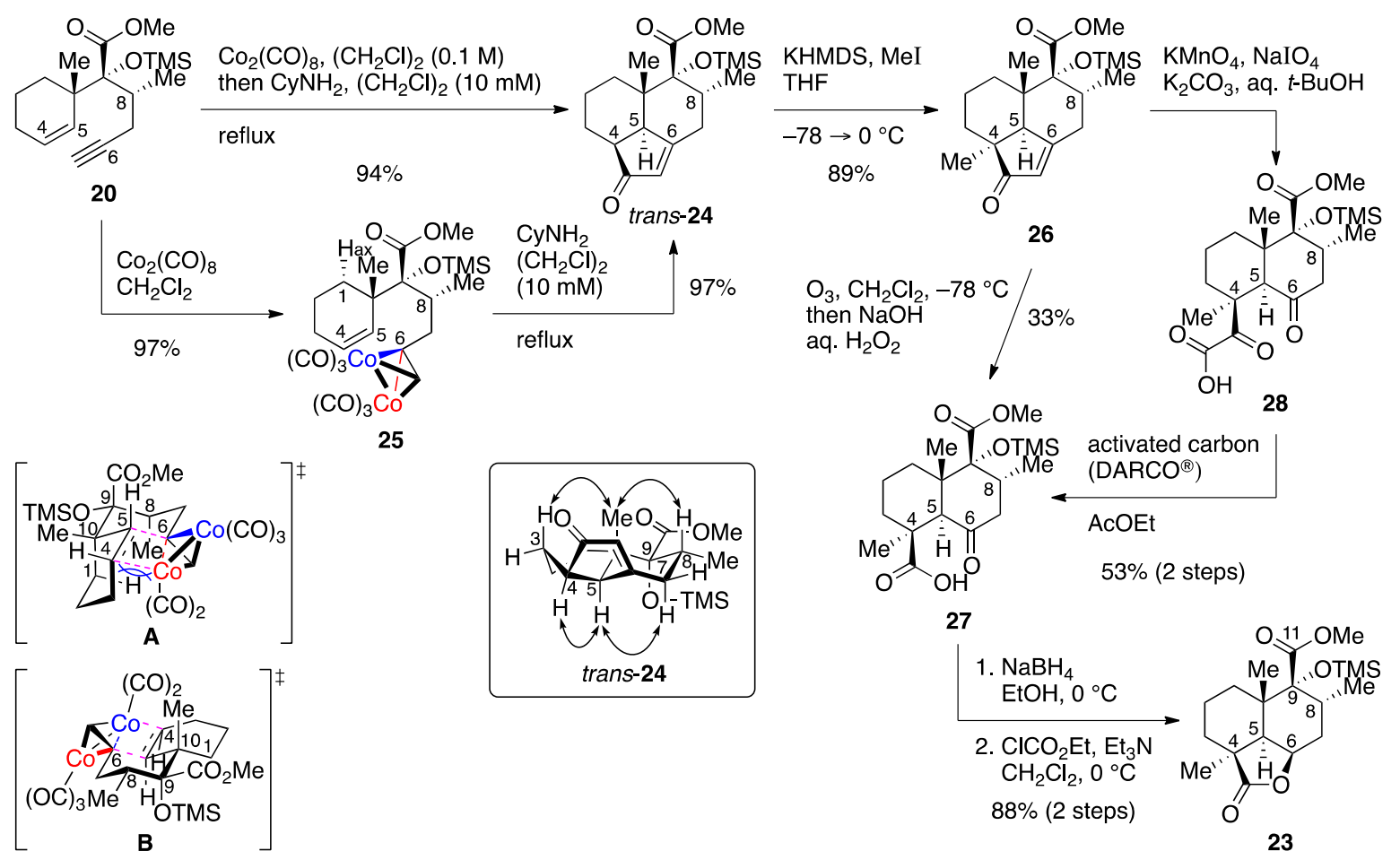

Scheme 2. Construction of the tricyclic lactone. KHMDS = potassium bis(trimethylsilyl)amide, THF $=$ tetrahydrofuran. 
To enhance the synthetic utility of PKR, a number of catalytic methods have been reported, and a variety of metal complexes have been employed for this purpose [29]. However, our attempts to carry out the catalytic reaction with enyne $\mathbf{2 0}$ met with failure: the use of Krafft conditions [30] resulted in recovery of enyne 20 in $49 \%$ yield, whereas a complicated mixture was obtained when using 2-naphthaldehyde as a $\mathrm{CO}$ donor in the $[\mathrm{RhCl}(\mathrm{cod})]_{2}$-catalyzed reaction [31]. In contrast to these unsuccessful results, the transformation of enyne 20 to enone trans-24 could be refined to a one-pot procedure, wherein the cobalt complex was formed in $\left(\mathrm{CH}_{2} \mathrm{Cl}\right)_{2}$ and heated under reflux after addition of $\mathrm{CyNH}_{2}$ and 10-fold dilution with $\left(\mathrm{CH}_{2} \mathrm{Cl}\right)_{2}$. As anticipated, alkylation of the potassium enolate generated from trans-24 with MeI in THF took place exclusively from the less-hindered $\alpha$-face to provide enone 26 in $89 \%$ yield.

With tricyclic compound $\mathbf{2 6}$ in hand, efforts were next focused on the transformation of the cyclopentenone moiety to the corresponding $\gamma$-butyrolactone. With regard to the oxidative cleavage of the cyclopentenone ring, it was found that desired $\gamma$-ketocarboxylic acid 27 could be obtained upon exposure of enone 26 to ozone in $\mathrm{CH}_{2} \mathrm{Cl}_{2}$ at $-78{ }^{\circ} \mathrm{C}$ followed by either reductive $\left(\mathrm{Me}_{2} \mathrm{~S}\right)$ or oxidative $\left(\mathrm{H}_{2} \mathrm{O}_{2}, \mathrm{NaOH}\right)$ workup [32], but the reaction suffered from low yield (33\%) and reproducibility issues. While $\alpha$-ketocarboxylic acid 28, detected as a byproduct, could be converged to $\gamma$-ketocarboxylic acid 27 upon treatment with $\mathrm{H}_{2} \mathrm{O}_{2}$ in aqueous $\mathrm{NaOH} / \mathrm{THF}$, no improvement in overall yield was observed. We then investigated a stepwise approach via $\alpha$-ketocarboxylic acid 28 . After an extensive screening of oxidants, the $\mathrm{KMnO}_{4} / \mathrm{NaIO}_{4}$ system proved to be effective for the conversion of enone 26 to 28. To our surprise, submission of crude $\alpha$-ketocarboxylic acid $\mathbf{2 8}$ to activated carbon for removal of the residual $\mathrm{Mn}$ salt to avoid decomposition of $\mathrm{H}_{2} \mathrm{O}_{2}$ effected desired decarbonylation. As a consequence, $\gamma$-ketocarboxylic acid 27 could be obtained in 53\% yield over two steps. The reason for the decarbonylation is unclear at present, but the possibility of involvement of contaminated Mn salt was excluded due to the fact that the reaction occurred from $\alpha$-ketocarboxylic acid $\mathbf{2 8}$ produced by ozonolysis [33]. To the best of our knowledge, this is the first example of activated carbon-mediated decarbonylation of $\alpha$-ketocarboxylic acid. Lactone formation from $\gamma$-ketocarboxylic acid 27 was achieved following the precedents of Wheeler [34] and Mangoni [19]: selective reduction of the carbonyl group at $\mathrm{C} 6$ with $\mathrm{NaBH}_{4}$ in $\mathrm{EtOH}$ at $0{ }^{\circ} \mathrm{C}$ was followed by lactonization through a mixed anhydride upon treatment with $\mathrm{ClCO}_{2} \mathrm{Et}$ in the presence of $\mathrm{Et}_{3} \mathrm{~N}$ in $\mathrm{CH}_{2} \mathrm{Cl}_{2}$ at $0{ }^{\circ} \mathrm{C}$ to give lactone 23 in $88 \%$ yield over two steps [35].

The remaining operation necessary for the synthesis of advanced intermediate 22 involved chemoselective reduction of the ester functionality in the presence of the lactone moiety (Scheme 3). As a prelude to the conversion, TMS ether 23 was converted to $\alpha$-hydroxyester 29 by exposure to $\mathrm{Bu}_{4} \mathrm{NF}$ in THF at $0{ }^{\circ} \mathrm{C}\left(98 \%\right.$ yield). With regard to the reduction, the use of $\mathrm{NaBH}_{4}$ or $\mathrm{LiBH}_{4}$ resulted in no reaction even at the reflux temperature, whereas the lactone moiety in $\mathbf{2 9}$ was selectively reduced with diisobutylaluminum hydride (DIBALH) in $\mathrm{CH}_{2} \mathrm{Cl}_{2}$ at $-78^{\circ} \mathrm{C}$. We were gratified to find that the desired chemoselective reduction could be achieved by the use of sodium bis(2-methoxyethoxy)aluminum hydride $\left(\operatorname{Red}-\mathrm{Al}^{\circledR}\right)$ as a reducing agent. It should be noted that the hydroxyl-directed reduction was accompanied by some lactone reduction when performed using $\mathrm{Et}_{2} \mathrm{O}$ or THF as a solvent or when performed at temperatures above $-20{ }^{\circ} \mathrm{C}$. The use of $\mathrm{CH}_{2} \mathrm{Cl}_{2}$ proved optimal in terms of chemoselectivity and solubility of substrate $\mathbf{2 9}$, but the collapse of the five-membered aluminate intermediate was retarded under the optimal conditions, resulting in the formation of a mixture of aldehyde 30 and 1,2-diol 31 after aqueous workup. Thus, the mixture needed to be subjected again to Red-Al ${ }^{\circledR}$ in $\mathrm{CH}_{2} \mathrm{Cl}_{2}$ at $-23{ }^{\circ} \mathrm{C}$ for the full reduction to 1,2-diol 31. The synthesis of advanced intermediate 22 was completed upon treatment of 1,2-diol 31 with $p$-toluenesulfonylimidazole and $\mathrm{NaH}$ in THF at $0{ }^{\circ} \mathrm{C}(84 \%$ yield over three steps $)$. 


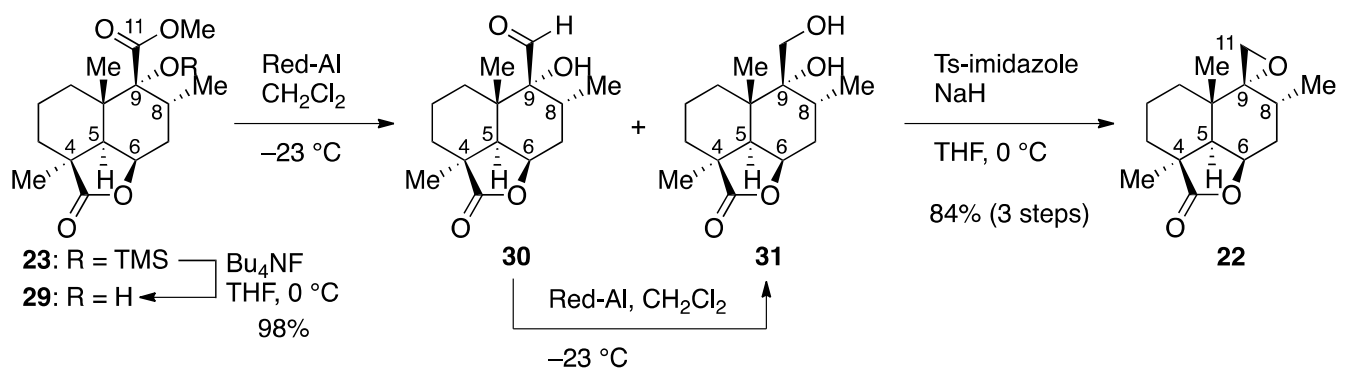

Scheme 3. Completion of the synthesis of advanced intermediate 22. Ts $=p$-toluenesulfonyl.

\subsection{Total Syntheses of Marrubiin and Related Labdane Diterpene Lactones}

Having established a route to epoxide 22, the stage was now set for elongation of the side chain for total syntheses. In this regard, Welch and co-workers reported the synthesis of isomarrubiin (C9-epi-marrubiin) by a CuI-catalyzed epoxide ring-opening reaction of the C9-epimer of epoxide 22 in $\mathrm{Et}_{2} \mathrm{O}$ at room temperature (40\% yield) [36]. With this reaction serving as a reference, we first examined $\mathrm{Cu}(\mathrm{I})$-catalyzed epoxide-opening reaction with Grignard reagent 32 [37] (Scheme 4). After some experimentation, we found that the use of a stoichiometric amount of $\mathrm{CuBr} \cdot \mathrm{SMe}_{2}$ was more effective, providing marrubiin $(\mathbf{1}),[\alpha]_{D}^{21}+34.4\left(c 1.04, \mathrm{CHCl}_{3}\right)\left[\right.$ [it. [38], $\left.[\alpha]_{D}^{20}+35.8\left(c 3.1, \mathrm{CHCl}_{3}\right)\right]$ in $65 \%$ yield. However, Grignard reagent 32 is prone to isomerization due to the high acidity of the furan 2-position, resulting in the formation of isomer 33 in 9\% yield [39]. This problem was circumvented by the use of Grignard reagent 34 [40], the TMS group of which was uneventfully removed with $\mathrm{Bu}_{4} \mathrm{NF}$ in THF [41] after the epoxide-opening reaction.

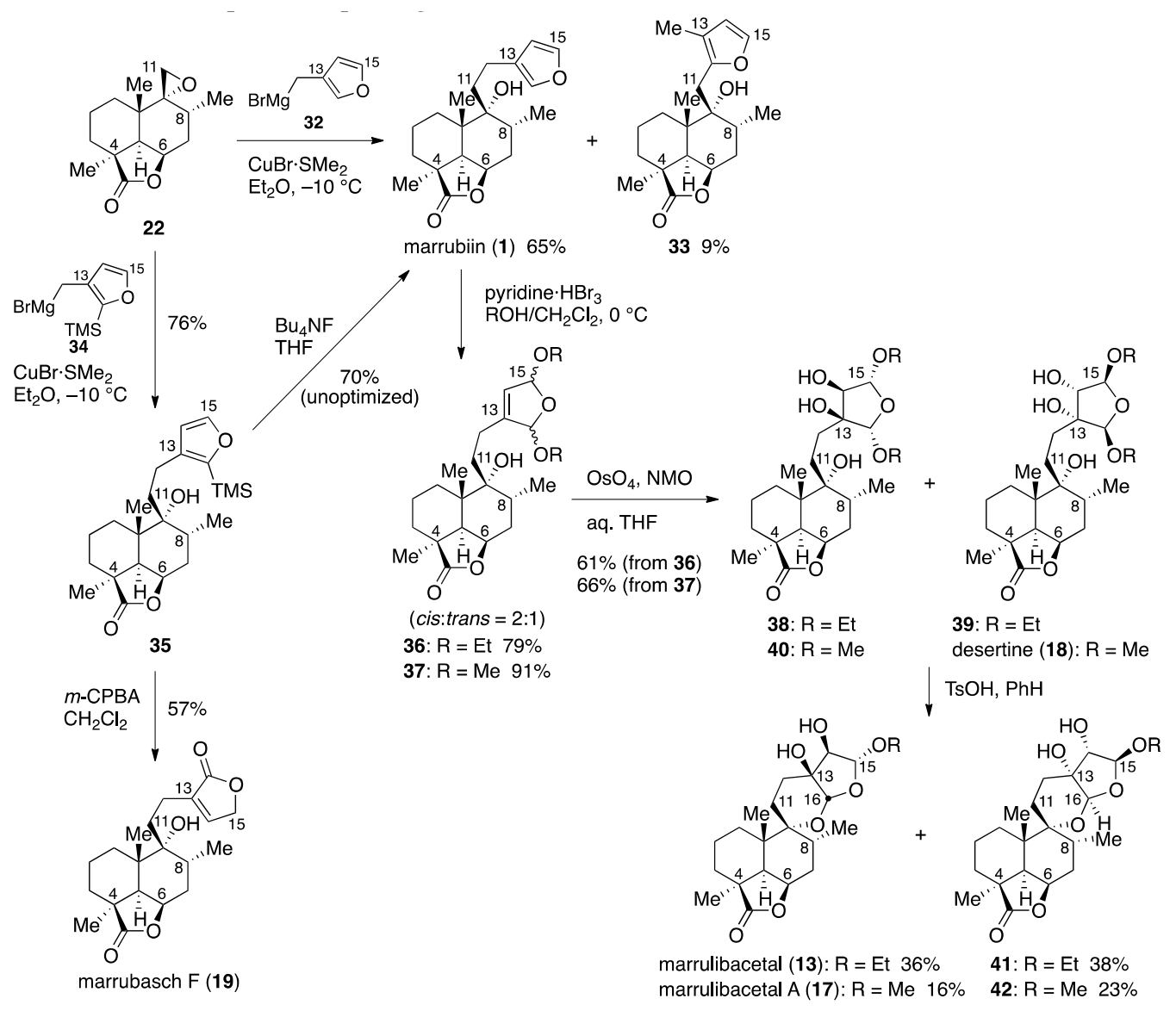

Scheme 4. Total syntheses of marrubiin (1), marrulibacetal (13), marrulibacetal A (17), and marrubasch F (19) from epoxide 22. 
An inspection of the structures of marrulibacetal (13) and marrulibacetal A (17) revealed that the highly oxidized tetrahydrofuran ring of these molecules could be formed by oxidation of the furan moiety in marrubiin (1) followed by internal acetalization. While unprecedented in the transformation of the marrubiin class labdane diterpenoids, successive oxidations of a furan ring have been documented in semi-synthesis of the neoclerodane diterpene natural products salvinicins A and B by Prisinzano and co-workers [42]. Furthermore, Frontana-Uribe and co-workers reported construction of the $[6,6,5,5]$-tetracyclic framework, in which 4,5,6,7a-tetrahydro-2H-furo[2,3- $b$ ]pyran is spirolinked to a trans-decalin ring system, by an electrochemical oxidation of hispanolone [43]. When marrubiin (1) was exposed to pyridine tribromide in $\mathrm{EtOH} / \mathrm{CH}_{2} \mathrm{Cl}_{2}$ at $0{ }^{\circ} \mathrm{C}$, oxidative acetalization occurred to give bisacetal 36 in 79\% yield with a cis/trans ratio of 2:1, albeit with no sign of internal acetalization in contrast to Frontana-Uribe's work. This result is attributed to the conformational constraint imposed by the lactone ring. The chemical yield was improved to $84 \%$ in the presence of $\mathrm{K}_{2} \mathrm{CO}_{3}$ as an acid scavenger. As expected from the precedent [44], the reaction rate of bisacetal 36 with $\mathrm{OsO}_{4} / 4$-methylmorpholine $\mathrm{N}$-oxide (NMO) in aqueous THF was influenced by the substrate structure: the reaction of a 1:1 mixture of diastereomers with the cis relative configuration at room temperature proceeded to completion within $1 \mathrm{~h}$ to give diols $\mathbf{3 8}$ and $\mathbf{3 9}$ in a stereoselective manner, whereas the trans-isomers were almost recovered unchanged under these conditions. Although the remaining trans-isomers could be consumed at an elevated temperature after prolonged reaction times, the lower $\pi$-facial selectivity (desired:undesired $=1: 1.2$ ), together with the fact that recovered trans-isomers could be isomerized to cis-isomers upon exposure to pyridinium $p$-toluenesulfonate (PPTS) in EtOH for $1 \mathrm{~h}$, prompted us to perform the reaction at room temperature. Submission of an inseparable mixture of diols 38 and 39 to $\mathrm{TsOH}$ in benzene effected internal transacetalization, affording marrulibacetal (13), $[\alpha]_{D}^{27}-21.7$ (c 1.16, $\left.\mathrm{CHCl}_{3}\right)$ [lit. [12], $[\alpha]_{D}^{25}-13.1$ ( $\left.\left.c 0.29, \mathrm{CHCl}_{3}\right)\right]$, in 36\% yield along with isomer 41 (38\% yield) and other diastereomers $(1: 1,18 \%$ combined yield). It is noteworthy that the dehydration of commercially available benzene with $3 \AA$ molecular sieves prior to use affected the transacetalization, leading to the decomposition of substrates, although the reason is unclear at present.

Following the same reaction sequence using $\mathrm{MeOH}$ instead of EtOH, marrulibacetal A (17), $[\alpha]_{D}^{21}-14.0\left(c 1.69, \mathrm{CHCl}_{3}\right)$ [lit. [14], $[\alpha]_{D}^{25}-10.77$ (the solvent and concentration were not reported)], could be synthesized from marrubiin (1). It should be mentioned that one of two diastereomers, obtained as an inseparable mixture in a ratio of $1: 1$ by dihydroxylation of bisacetal 37 , matched by ${ }^{1} \mathrm{H}-\mathrm{NMR}$ with desertine (18), although the chemical correlation to establish the stereochemistry will be presented later (vide infra). The low chemical yield (16\%) of $\mathbf{1 7}$ was due to the formation of two isomers with the trans $\mathrm{H} 15 / \mathrm{H} 16$ stereochemistry in $38 \%$ yield.

Kuwajima and Urabe reported that 2-(trimethylsilyl)furans could be oxidized regioselectively with peracetic acid [45], and the method was successfully applied for the synthesis of substituted $\Delta^{2}$-butenolides by Goldsmith, Liotta, and co-workers [46]. On the basis of these precedents, we next examined the oxidation of 2-(trimethylsilyl)furan 35 for the conversion to marrubasch F (19). Screening of peracids revealed that the use of $m$-chloroperoxybenzoic acid ( $m$-CPBA) (57\% yield) and peracetic acid (52\% yield) afforded 19, mp $195-196{ }^{\circ} \mathrm{C},[\alpha]_{D}^{21}+41.1\left(c 0.53, \mathrm{CHCl}_{3}\right)$ [lit. [20], mp 191-193 ${ }^{\circ} \mathrm{C}$, $\left.[\alpha]_{D}+41.5\left(c 1.00, \mathrm{CHCl}_{3}\right)\right]$, with $m$-CPBA being the optimal oxidizing agent, whereas only desilylation occurred to give marrubiin (1) with either performic acid or magnesium monoperoxyphthalate (MMPP).

Having synthesized marrubiin (1) and natural products possessing the same carbon framework in a higher oxidation state, we next addressed the conversion of epoxide 22 to natural products 12 and 14, which required two- and three-carbon nucleophiles, respectively (Scheme 5). Fortunately, epoxide 22 underwent nucleophilic ring-opening reaction with commercially available lithium acetylide ethylenediamine complex, affording alcohol 43 in 95\% yield. Oxidative lactonization of homopropargyl alcohol 43 could be attained by the gold(I)-catalyzed cycloisomerization/oxidation sequence under Ye conditions [47], completing the synthesis of cyllenine C (12). On the other hand, the use of Grignard reagent 44 [48] as a three-carbon nucleophile under conditions identical to those with 32 gave alcohol 45 (48\% yield), which was desilylated with $\mathrm{Bu}_{4} \mathrm{NF}$ in THF to furnish 1,5-diol 46 in $82 \%$ yield. 
$\delta$-Lactone could be formed upon oxidation of 1,5-diol 46 with 2,2,6,6-tetramethylpiperidin-1-oxyl (TEMPO)/PhI(OAc) $)_{2}$ in $\mathrm{CH}_{2} \mathrm{Cl}_{2}$ according to the Forsyth protocol [49] to give marrulactone (14) in 91\% yield. Since the $\gamma$-lactone in $\mathbf{1 4}$ proved more reluctant to hydrolyze, regioselective saponification could be realized with $\mathrm{LiOH}$ in THF, providing marrulanic acid (10) in $84 \%$ yield. Although synthetic compounds 12, 14, and 10 would be identical to natural products as judged by ${ }^{1} \mathrm{H}$ and ${ }^{13} \mathrm{C}-\mathrm{NMR}$ analysis (see Supplementary Materials), their specific rotations $\left[[\alpha]_{D}^{24}+22.1\left(c 0.69, \mathrm{CH}_{2} \mathrm{Cl}_{2}\right),[\alpha]_{D}^{21}\right.$ - $11.6\left(c 0.61, \mathrm{CHCl}_{3}\right)$, and $[\alpha]_{D}^{27}+25.4\left(c 0.53, \mathrm{CHCl}_{3}\right)$, respectively] were inconsistent with those observed for natural cyllenine $C(\mathbf{1 2})$, marrulactone (14), and marrulanic acid $(\mathbf{1 0})\left[[\alpha]_{D}^{20}+11.82(c\right.$ $\left.0.33, \mathrm{CH}_{2} \mathrm{Cl}_{2}\right)[11],[\alpha]_{D}^{25}-23.80\left(c 0.22, \mathrm{CHCl}_{3}\right)$ [12], and $[\alpha]_{D}^{25}-10.8\left(c 1.2, \mathrm{CHCl}_{3}\right)$ [12], respectively]. This result is attributed to contamination of the impurity in these natural products, as detected by NMR spectroscopy.

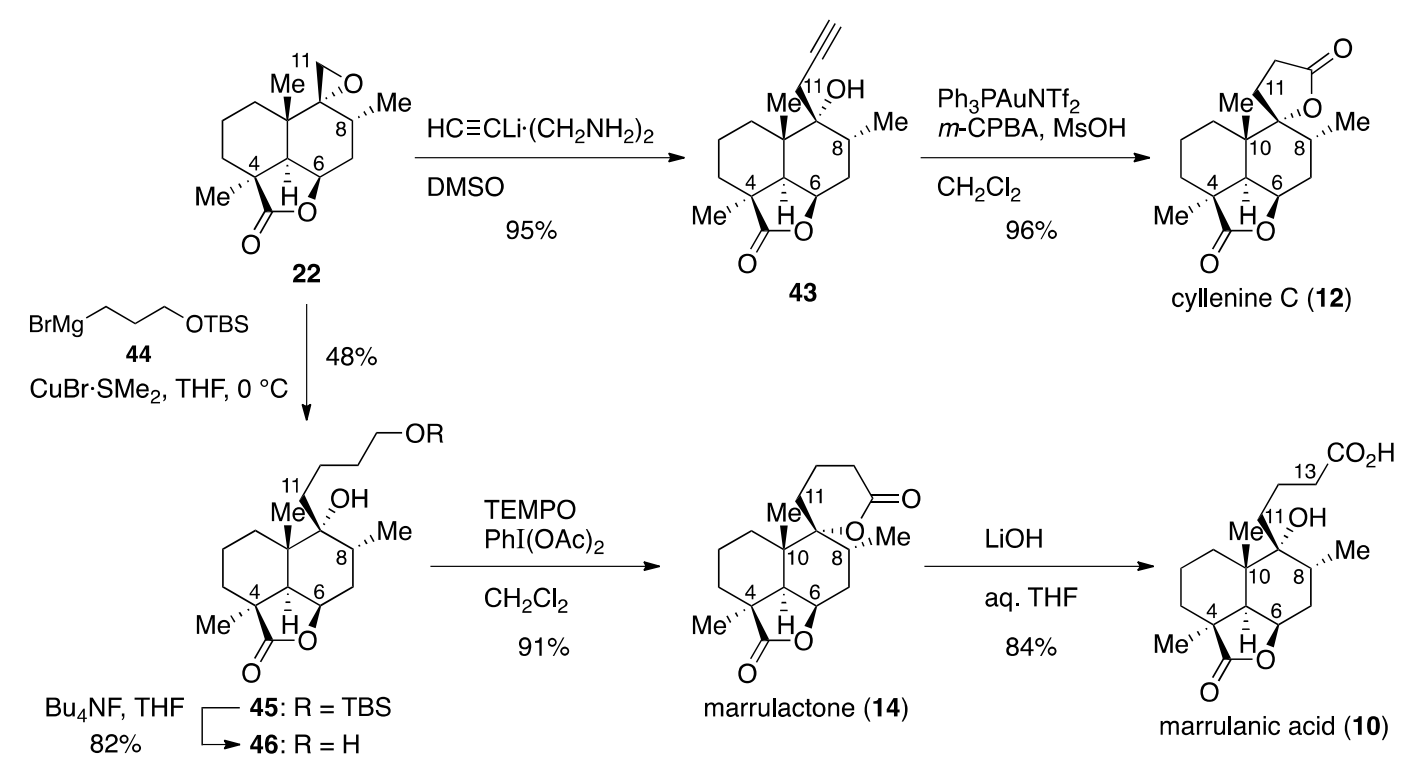

Scheme 5. Total syntheses of cyllenine C (12), marrulactone (14), and marrulanic acid (10) from epoxide 22. DMSO = dimethyl sulfoxide, $\mathrm{Tf}$ = trifluoromethanesulfonyl, $\mathrm{Ms}=$ methanesulfonyl, $\mathrm{TBS}=$ tert-butyldimethylsilyl.

Of the eight natural products synthesized, desertine (18) could not be purified due to the difficulty of separation from its diastereomer 40. With regard to this natural product, stereochemistries were determined by an NOESY experiment by Dijoux-Franca and co-workers, who indicated two structures differing in the configuration at C15 [14]. Furthermore, evidence that supports the stereochemical relationship between the decalin and tetrahydrofuran moieties, separated by the $\mathrm{C} 11-\mathrm{C} 12$ two-carbon bridge, was not provided in their report. Therefore, we felt compelled to perform experiments to determine the stereochemistries of $\mathbf{1 8}$, and we found that exposure of marrulibacetal A (17) and its diastereomer 42 to $\mathrm{TsOH}$ in refluxing $\mathrm{MeOH}$ to effect transacetalization resulted in the formation of triol 40 and desertine (18), respectively, albeit in low yields (Scheme 6). These results suggest that the stereocenters at $\mathrm{C} 13$ and $\mathrm{C} 14$ of $\mathbf{1 8}$ have configurations opposite to those of $\mathbf{1 7}$. Together with our previous observation that desertine was produced from cis-37, the stereochemistries of this natural product can be established as shown for $\mathbf{1 8}$. 


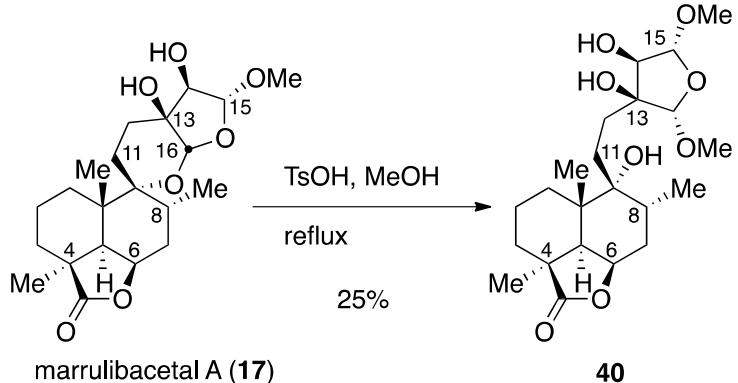

marrulibacetal $A(\mathbf{1 7})$

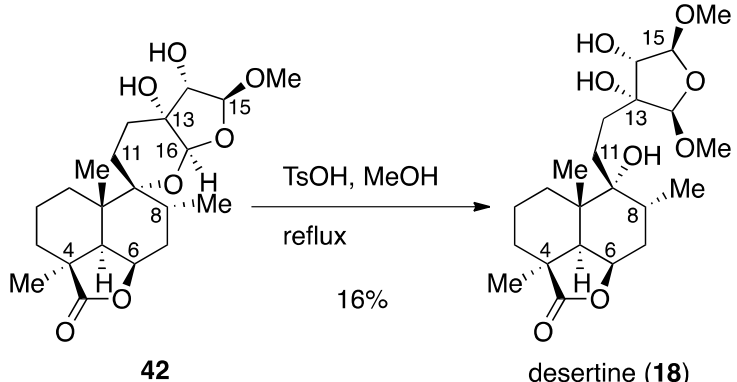

42

Scheme 6. Conversion of marrulibacetal A (17) and its isomer 42 to triol 40 and desertine (18).

\section{Materials and Methods}

\section{Chemistry}

\subsubsection{General Information}

Optical rotations were recorded on a digital polarimeter with a sodium lamp (589 nm). Infrared (IR) spectra were recorded on an FT-IR spectrophotometer and absorbance bands are reported in wavenumber $\left(\mathrm{cm}^{-1}\right)$. Proton nuclear magnetic resonance $\left({ }^{1} \mathrm{H}-\mathrm{NMR}\right)$ spectra were recorded with tetramethylsilane $\left(\delta_{\mathrm{H}} 0.00\right), \mathrm{CHCl}_{3}\left(\delta_{\mathrm{H}} 7.26\right)$, or $\mathrm{CH}_{2} \mathrm{Cl}_{2}\left(\delta_{\mathrm{H}} 5.32\right)$ as an internal standard. Coupling constants $(J)$ are reported in hertz $(\mathrm{Hz})$. Abbreviations of multiplicity are as follows: s, singlet; $\mathrm{d}$, doublet; $\mathrm{t}$, triplet; $\mathrm{q}$, quartet; $\mathrm{m}$, multiplet; br, broad. Data are presented as follows: chemical shift, multiplicity, coupling constants, integration, and assignment. Marrulibacatal numbering is used for proton assignments of all intermediates. Carbon nuclear magnetic resonance $\left({ }^{13} \mathrm{C}-\mathrm{NMR}\right)$ spectra were recorded with $\mathrm{CDCl}_{3}\left(\delta_{\mathrm{C}} 77.0\right), \mathrm{CD}_{2} \mathrm{Cl}_{2}\left(\delta_{\mathrm{C}} 53.84\right)$, or acetone- $d_{6}\left(\delta_{\mathrm{C}} 29.84\right)$ as an internal standard. High-resolution mass spectra (HRMS) were recorded either by electrospray ionization (ESI) using a time-of-flight (TOF) analyzer, or by electron impact (EI) using a magnetic sector analyzer. Column chromatography was carried out on silica gel $60 \mathrm{~N}(63-210 \mu \mathrm{m}$ or 40-50 $\mu \mathrm{m}$ ). Analytical thin layer chromatography (TLC) was carried out with $0.25 \mathrm{~mm}$ silica gel plates. Visualization was accomplished with ultraviolet light and anisaldehyde stain, followed by heating. Reagents and solvents were purified by standard means or used as received unless otherwise noted. Dehydrated dichloromethane $\left(\mathrm{CH}_{2} \mathrm{Cl}_{2}\right)$ and tetrahydrofuran (THF, stabilizer free) were purchased. Cyclohexylamine was distilled from calcium hydride. $4 \AA$ molecular sieves was finely ground in mortar and heated in vacuo at $180{ }^{\circ} \mathrm{C}$ for $4 \mathrm{~h} . p$-Toluenesulfonylimidazole [50], (3-furylmethyl)magnesium bromide [37], $\mathrm{CuBr} \cdot \mathrm{SMe}_{2}$ [51], [2-(trimethylsilyl)furan-3-yl]methanol [52], and 3-(tert-butyldimethylsilyl)oxypropylmagnesium bromide (44) [48] were prepared according to literature procedures. All reactions were conducted under an argon atmosphere unless otherwise noted.

\subsubsection{Experimental Procedures and Compound Data}

Methyl [2R,2(1S),3R]-3-methyl-2-(1-methylcyclohex-2-en-1-yl)-2-(trimethylsilyl)oxyhex-5-ynoate dicobalt hexacarbonyl complex (25). $\mathrm{Co}_{2}(\mathrm{CO})_{8}(663 \mathrm{mg}, 1.94 \mathrm{mmol})$ was added to an ice-cooled $\left(0^{\circ} \mathrm{C}\right)$ solution of enyne $20(522 \mathrm{mg}, 1.62 \mathrm{mmol})$ in $\mathrm{CH}_{2} \mathrm{Cl}_{2}(16 \mathrm{~mL})$. After $1.5 \mathrm{~h}$ of stirring at room temperature, the reaction mixture was concentrated in vacuo, and the reddish brown residue was chromatographed twice (silica gel $38 \mathrm{~g}, 20: 1 \mathrm{n}$-hexane/AcOEt) to give dicobalt complex 25 (958 mg, 97\%) as a dark red oil. $R_{f} 0.51$ (20:1 n-hexane/AcOEt); IR (neat) 2951, 2091, 2048, 2016, 1748, 1250, 1175, 1138, $841 \mathrm{~cm}^{-1}$; ${ }^{1} \mathrm{H}-\mathrm{NMR}\left(500 \mathrm{MHz}, \mathrm{CDCl}_{3}\right) \delta 0.19\left(\mathrm{~s}, 9 \mathrm{H}, \mathrm{Si}\left(\mathrm{CH}_{3}\right)_{3}\right), 0.87\left(\mathrm{~d}, J=6.6 \mathrm{~Hz}, 3 \mathrm{H}, \mathrm{C} 8-\mathrm{CH}_{3}\right), 1.00(\mathrm{~s}, 3 \mathrm{H}$, $\left.\mathrm{C} 10-\mathrm{CH}_{3}\right), 1.42\left(\mathrm{br} \mathrm{d}, J=13.8 \mathrm{~Hz}, 1 \mathrm{H}\right.$, one of $\left.\mathrm{C} 1-\mathrm{H}_{2}\right), 1.63\left(\mathrm{~m}, 1 \mathrm{H}\right.$, one of $\left.\mathrm{C} 2-\mathrm{H}_{2}\right), 1.71(\mathrm{~m}, 1 \mathrm{H}$, one of $\left.\mathrm{C} 2-\mathrm{H}_{2}\right), 1.89-2.03\left(\mathrm{~m}, 3 \mathrm{H}\right.$, one of $\left.\mathrm{C} 1-H_{2}, \mathrm{C} 3-\mathrm{H}_{2}\right), 2.28(\mathrm{ddq}, J=2.7,11.4,6.6 \mathrm{~Hz}, 1 \mathrm{H}, \mathrm{C} 8-\mathrm{H}), 2.60(\mathrm{ddd}, J$ $=1.0,11.4,14.7 \mathrm{~Hz}, 1 \mathrm{H}$, one of $\left.\mathrm{C} 7-\mathrm{H}_{2}\right), 3.49\left(\mathrm{br} \mathrm{d}, J=14.7 \mathrm{~Hz}, 1 \mathrm{H}\right.$, one of $\left.\mathrm{C} 7-\mathrm{H}_{2}\right), 3.71\left(\mathrm{~s}, 3 \mathrm{H}, \mathrm{CO}_{2} \mathrm{CH}_{3}\right)$, $5.71(\mathrm{ddd}, J=1.7,5.2,10.2 \mathrm{~Hz}, 1 \mathrm{H}, \mathrm{C} 4-H), 5.82(\mathrm{br} \mathrm{d}, J=10.2 \mathrm{~Hz}, 1 \mathrm{H}, \mathrm{C} 5-H), 6.01$ (br t, $J=1.0 \mathrm{~Hz}, 1 \mathrm{H}$, 
C6-CH) ${ }^{13} \mathrm{C}-\mathrm{NMR}\left(125.7 \mathrm{MHz}, \mathrm{CDCl}_{3}\right) \delta 2.8\left(\mathrm{CH}_{3}\right), 16.1\left(\mathrm{CH}_{3}\right), 20.0\left(\mathrm{CH}_{2}\right), 24.4\left(\mathrm{CH}_{2}\right), 25.1\left(\mathrm{CH}_{3}\right), 31.5$ $\left(\mathrm{CH}_{2}\right), 36.9\left(\mathrm{CH}_{2}\right), 41.3(\mathrm{CH}), 42.4(\mathrm{C}), 51.5\left(\mathrm{CH}_{3}\right), 74.2(\mathrm{C}), 88.5(\mathrm{C}), 95.8(\mathrm{CH}), 126.8(\mathrm{CH}), 132.8(\mathrm{CH})$, $175.1(\mathrm{C}), 200.0(\mathrm{C})$.

Methyl (1S,6R,7R,8S,12R)-6,8-dimethyl-2-oxo-7-(trimethylsilyl)oxytricyclo[6.3.1.0 4,12]dodec-3-ene-7-carboxylate (trans-24). A solution of dicobalt complex $25(150 \mathrm{mg}, 0.247 \mathrm{mmol})$ in 1,2-dichloroethane (5 mL plus $2 \times 2.5 \mathrm{~mL}$ rinse) was added to a refluxing solution of cyclohexylamine $(0.17 \mathrm{~mL}, 1.49 \mathrm{mmol}) \mathrm{in}$ 1,2-dichloroethane $(15 \mathrm{~mL})$, and the resulting mixture was refluxed for $1.5 \mathrm{~h}$. After cooling, the mixture was filtered through a Celite pad, and the filtrate was concentrated in vacuo. Purification of the residue (503 $\mathrm{mg}$ ) by column chromatography (silica gel $30 \mathrm{~g}, 3: 1 \mathrm{n}$-hexane/AcOEt) gave enone trans-24 $(84.0 \mathrm{mg}, 97 \%)$ as a white solid. $R_{f} 0.42$ (3:1 $n$-hexane/AcOEt); mp $75-76{ }^{\circ} \mathrm{C}$ (colorless plates from n-hexane); $[\alpha]_{D}^{25}+62.1$ (c 1.34, $\mathrm{CHCl}_{3}$ ); IR (KBr) 2945, 1736, 1694, 1620, 1458, 1260, 1184, 1096, 1034, 982, $843 \mathrm{~cm}^{-1} ;{ }^{1} \mathrm{H}-\mathrm{NMR}\left(500 \mathrm{MHz}, \mathrm{CD}_{2} \mathrm{Cl}_{2}\right) \delta 0.20\left(\mathrm{~s}, 9 \mathrm{H}, \mathrm{Si}\left(\mathrm{CH}_{3}\right)_{3}\right), 0.62\left(\mathrm{~s}, 3 \mathrm{H}, \mathrm{C} 10-\mathrm{CH}_{3}\right), 0.91(\mathrm{~d}, \mathrm{~J}=6.2$ $\left.\mathrm{Hz}, 3 \mathrm{H}, \mathrm{C} 8-\mathrm{CH}_{3}\right), 1.01\left(\mathrm{dt}, \mathrm{J}=13.4,7.4 \mathrm{~Hz}, 1 \mathrm{H}\right.$, one of $\left.\mathrm{C} 1-\mathrm{H}_{2}\right), 1.51-1.63\left(\mathrm{~m}, 2 \mathrm{H}\right.$, one of $\mathrm{C} 2-\mathrm{H}_{2}$, one of $\left.\mathrm{C} 3-\mathrm{H}_{2}\right), 1.69\left(\mathrm{~m}, 1 \mathrm{H}\right.$, one of $\left.\mathrm{C} 2-\mathrm{H}_{2}\right), 1.80\left(\mathrm{~m}, 1 \mathrm{H}\right.$, one of $\left.\mathrm{C} 3-\mathrm{H}_{2}\right), 1.89(\mathrm{ddd}, J=4.5,8.9,13.4 \mathrm{~Hz}, 1 \mathrm{H}$, one of $\left.\mathrm{C} 1-\mathrm{H}_{2}\right), 2.16\left(\mathrm{t}, J=13.9 \mathrm{~Hz}, 1 \mathrm{H}\right.$, one of $\left.\mathrm{C} 7-\mathrm{H}_{2}\right), 2.47(\mathrm{ddd}, J=7.3,8.5,9.5 \mathrm{~Hz}, 1 \mathrm{H}, \mathrm{C} 4-\mathrm{H}), 2.56-2.61(\mathrm{~m}$, $2 \mathrm{H}$, one of $\left.\mathrm{C} 7-\mathrm{H}_{2}, \mathrm{C} 8-\mathrm{H}\right), 3.16(\mathrm{~d}, \mathrm{~J}=7.3 \mathrm{~Hz}, 1 \mathrm{H}, \mathrm{C} 5-\mathrm{H}), 3.70\left(\mathrm{~s}, 3 \mathrm{H}, \mathrm{CO}_{2} \mathrm{CH}_{3}\right), 5.80(\mathrm{~s}, 1 \mathrm{H},=\mathrm{CHCO})$; ${ }^{13} \mathrm{C}-\mathrm{NMR}\left(125.7 \mathrm{MHz}, \mathrm{CD}_{2} \mathrm{Cl}_{2}\right) \delta 2.6\left(\mathrm{CH}_{3}\right), 18.3\left(\mathrm{CH}_{3}\right), 19.7\left(\mathrm{CH}_{2}\right), 19.9\left(\mathrm{CH}_{2}\right), 21.1\left(\mathrm{CH}_{3}\right), 28.8\left(\mathrm{CH}_{2}\right)$, $34.9\left(\mathrm{CH}_{2}\right), 37.0(\mathrm{CH}), 43.7(\mathrm{CH}), 45.0(\mathrm{CH}), 45.8(\mathrm{C}), 51.8\left(\mathrm{CH}_{3}\right), 86.1(\mathrm{C}), 126.0(\mathrm{CH}), 172.9(\mathrm{CH}), 179.4$ (C), 211.9 (C); HRMS (ESI) $\mathrm{m} / z$ [M + Na ${ }^{+}$calcd for $\mathrm{C}_{19} \mathrm{H}_{30} \mathrm{O}_{4} \mathrm{SiNa}$ 373.1806; found 373.1823.

One-pot reaction. $\mathrm{Co}_{2}(\mathrm{CO})_{8}(4.08 \mathrm{~g}, 11.9 \mathrm{mmol})$ was added to an ice-cooled $\left(0{ }^{\circ} \mathrm{C}\right)$ solution of enyne $20(3.20 \mathrm{~g}, 9.93 \mathrm{mmol})$ in 1,2-dichloroethane $(100 \mathrm{~mL})$, and the mixture was stirred at room temperature for $3 \mathrm{~h}$. The resulting dark red suspension was diluted with 1,2-dichloroethane (900 $\mathrm{mL})$, and cyclohexylamine $(8.0 \mathrm{~mL}, 70.7 \mathrm{mmol})$ was added. After $5 \mathrm{~h}$ of heating at reflux, the reaction mixture was cooled to room temperature, and the volatile elements were removed in vacuo. The residue was suspended in 4:1 n-hexane/AcOEt and filtered through a Celite pad. Evaporation of the filtrate in vacuo furnished the crude product $(5.75 \mathrm{~g})$, which was purified by column chromatography (silica gel $200 \mathrm{~g}, 25: 1 \rightarrow 10: 1 \rightarrow 4: 1 \rightarrow 1: 1 n$-hexane/AcOEt) to give enone trans-24 (3.26 g, 94\%) as a white solid.

Methyl (1S,6R,7R,8S,12R)-1,6,8-trimethyl-2-oxo-7-(trimethylsilyl)oxytricyclo[6.3.1.0 $\left.{ }^{4,12}\right]$ dodec-3-ene-7carboxylate (26). KHMDS in toluene $(0.5 \mathrm{M}, 6.2 \mathrm{~mL}, 3.10 \mathrm{mmol})$ was added to a cooled $\left(-78^{\circ} \mathrm{C}\right)$ solution of enone trans-24 $(988 \mathrm{mg}, 2.82 \mathrm{mmol})$ in THF $(30 \mathrm{~mL})$. After $40 \mathrm{~min}$ of stirring, iodomethane $(0.23 \mathrm{~mL}$, $3.69 \mathrm{mmol}$ ) was added, and the resulting mixture was stirred at $0{ }^{\circ} \mathrm{C}$ for $40 \mathrm{~min}$. The reaction was quenched with saturated aqueous $\mathrm{NH}_{4} \mathrm{Cl}(30 \mathrm{~mL})$, and the mixture was extracted with AcOEt $(3 \times 60$ $\mathrm{mL})$. The combined organic extracts were washed with brine $(2 \times 30 \mathrm{~mL})$, and dried over anhydrous $\mathrm{Na}_{2} \mathrm{SO}_{4}$. Filtration and evaporation in vacuo furnished the crude product $(1.16 \mathrm{~g})$, which was purified by column chromatography (silica gel $30 \mathrm{~g}, 6: 1 n$-hexane/AcOEt) to give methylated product 26 (915 $\mathrm{mg}, 89 \%)$ as a yellow oil. $R_{f} 0.40(4: 1 n \text {-hexane/AcOEt); } \alpha]_{D}^{22}+72.2\left(c 0.95, \mathrm{CHCl}_{3}\right)$; IR (neat) 3433, 2953, $1738,1703,1624,1458,1252,1186,1103,1042,841 \mathrm{~cm}^{-1} ;{ }^{1} \mathrm{H}-\mathrm{NMR}\left(500 \mathrm{MHz}, \mathrm{CDCl}_{3}\right) \delta 0.20(\mathrm{~s}, 9 \mathrm{H}$, $\left.\mathrm{Si}\left(\mathrm{CH}_{3}\right)_{3}\right), 0.71\left(\mathrm{~s}, 3 \mathrm{H}, \mathrm{C} 10-\mathrm{CH}_{3}\right), 0.91\left(\mathrm{~d}, \mathrm{~J}=6.3 \mathrm{~Hz}, 3 \mathrm{H}, \mathrm{C} 8-\mathrm{CH}_{3}\right), 1.02(\mathrm{ddd}, J=7.4,9.2,13.5 \mathrm{~Hz}, 1 \mathrm{H}$, one of $\left.\mathrm{C} 1-\mathrm{H}_{2}\right), 1.11\left(\mathrm{~s}, 3 \mathrm{H}, \mathrm{C} 4-\mathrm{CH}_{3}\right), 1.43\left(\mathrm{~m}, 1 \mathrm{H}\right.$, one of $\left.\left(\mathrm{CH}_{2}\right)_{2}\right), 1.60\left(\mathrm{~m}, 1 \mathrm{H}\right.$, one of $\left.\left(\mathrm{CH}_{2}\right)_{2}\right), 1.64-1.75$ $\left(\mathrm{m}, 2 \mathrm{H}\right.$, two of $\left.\left(\mathrm{CH}_{2}\right)_{2}\right), 1.92\left(\mathrm{ddd}, \mathrm{J}=2.8,10.2,13.5 \mathrm{~Hz}, 1 \mathrm{H}\right.$, one of $\left.\mathrm{C} 1-\mathrm{H}_{2}\right), 2.12\left(\mathrm{~m}, 1 \mathrm{H}\right.$, one of $\left.\mathrm{C} 7-\mathrm{H}_{2}\right)$, 2.54-2.62 (m, 2H, one of $\left.\mathrm{C} 7-\mathrm{H}_{2}, \mathrm{C} 8-\mathrm{H}\right), 2.67($ br s, $1 \mathrm{H}, \mathrm{C} 5-\mathrm{H}), 3.73\left(\mathrm{~s}, 3 \mathrm{H}, \mathrm{CO}_{2} \mathrm{CH}_{3}\right), 5.78(\mathrm{t}, \mathrm{J}=1.4$ $\mathrm{Hz}, 1 \mathrm{H},=\mathrm{CHCO}) ;{ }^{13} \mathrm{C}-\mathrm{NMR}\left(125.7 \mathrm{MHz}, \mathrm{CDCl}_{3}\right) \delta 2.5\left(\mathrm{CH}_{3}\right), 17.7\left(\mathrm{CH}_{2}\right), 18.0\left(\mathrm{CH}_{3}\right), 21.1\left(\mathrm{CH}_{3}\right), 26.6$ $\left(\mathrm{CH}_{2}\right), 27.3\left(\mathrm{CH}_{3}\right), 28.0\left(\mathrm{CH}_{2}\right), 34.8\left(\mathrm{CH}_{2}\right), 36.4(\mathrm{CH}), 45.80(\mathrm{C}), 45.84(\mathrm{C}), 51.5\left(\mathrm{CH}_{3}\right), 52.3(\mathrm{CH}), 85.7(\mathrm{C})$, $123.8(\mathrm{CH}), 172.6(\mathrm{C}), 178.5(\mathrm{C}), 215.8(\mathrm{C})$; HRMS (ESI) $m / z[\mathrm{M}+\mathrm{Na}]^{+}$calcd for $\mathrm{C}_{20} \mathrm{H}_{32} \mathrm{O}_{4} \mathrm{SiNa} 387.1962$; found 387.1950 .

(1S,4aS,5R,6R,8aR)-5-(Methoxycarbonyl)-1,4a,6-trimethyl-8-oxo-5-(trimethylsilyl)oxydecahydronaphthalene1-carboxylic acid (27). $\mathrm{KMnO}_{4}(4.6 \mathrm{mg}, 29.1 \mu \mathrm{mol})$ was added to a solution of $\mathrm{NaIO}_{4}(270 \mathrm{mg}, 1.26$ $\mathrm{mmol})$ in $\mathrm{H}_{2} \mathrm{O}(7.0 \mathrm{~mL})$, and the mixture was stirred for $30 \mathrm{~min}$. To the mixture was added $\mathrm{K}_{2} \mathrm{CO}_{3}(23.0$ 
$\mathrm{mg}, 0.166 \mathrm{mmol})$, followed by a solution of enone 26 ( $50.3 \mathrm{mg}, 0.138 \mathrm{mmol})$ in $t-\mathrm{BuOH}(1.4 \mathrm{~mL}$ plus $2 \times$ $1 \mathrm{~mL}$ rinse). After $24 \mathrm{~h}$ of stirring, the reaction was quenched with $\mathrm{NaHSO}_{3}(507 \mathrm{mg}, 4.87 \mathrm{mmol}$ ), and the resulting mixture was extracted with AcOEt $(3 \times 20 \mathrm{~mL})$. The combined organic extracts were washed with brine $(2 \times 15 \mathrm{~mL})$, and dried over anhydrous $\mathrm{Na}_{2} \mathrm{SO}_{4}$. Filtration and evaporation in vacuo furnished the crude product $(70.9 \mathrm{mg})$, which was used without further purification.

Activated carbon $(708 \mathrm{mg})$ was added to an ice-cooled $\left(0^{\circ} \mathrm{C}\right)$ solution of the crude $\alpha$-ketocarboxylic acid $28(70.9 \mathrm{mg})$ in AcOEt $(14 \mathrm{~mL})$. After $63 \mathrm{~h}$ of stirring at room temperature, the resulting suspension was filtered through a Celite pad, and the filtrate was evaporated in vacuo. Purification of the crude product $(74.7 \mathrm{mg}$ ) by column chromatography (silica gel $5.2 \mathrm{~g}$, 7:1 $n$-hexane/AcOEt) gave $\gamma$-ketocarboxylic acid $27(28.3 \mathrm{mg}, 53 \%)$ as an amorphous solid. $R_{f} 0.60\left(1: 1 n\right.$-hexane/AcOEt); $[\alpha]_{D}^{21}+$ 62.5 (c 1.01, $\mathrm{CHCl}_{3}$ ); IR (neat) 2953, 1732, 1717, 1674, 1456, 1252, 1182, 1153, 1090, $843 \mathrm{~cm}^{-1} ;{ }^{1} \mathrm{H}-\mathrm{NMR}$ $\left(500 \mathrm{MHz}, \mathrm{CDCl}_{3}\right) \delta 0.24\left(\mathrm{~s}, 9 \mathrm{H}, \mathrm{Si}\left(\mathrm{CH}_{3}\right)_{3}\right), 0.82\left(\mathrm{dt}, J=3.5,13.4 \mathrm{~Hz}, 1 \mathrm{H}\right.$, one of $\left.\mathrm{C} 3-\mathrm{H}_{2}\right), 0.88(\mathrm{~d}, J=6.4$ $\left.\mathrm{Hz}, 3 \mathrm{H}, \mathrm{C} 8-\mathrm{CH}_{3}\right), 0.97\left(\mathrm{~s}, 3 \mathrm{H}, \mathrm{C} 10-\mathrm{CH}_{3}\right), 1.04\left(\mathrm{~d}, \mathrm{~J}=13.2 \mathrm{~Hz}, 1 \mathrm{H}\right.$, one of $\left.\mathrm{C} 1-\mathrm{H}_{2}\right), 1.29\left(\mathrm{~s}, 3 \mathrm{H}, \mathrm{C} 4-\mathrm{CH}_{3}\right)$, $1.51\left(\mathrm{~m}, 1 \mathrm{H}\right.$, one of $\left.\mathrm{C} 2-\mathrm{H}_{2}\right), 1.85\left(\mathrm{~m}, 1 \mathrm{H}\right.$, one of $\left.\mathrm{C} 2-\mathrm{H}_{2}\right), 1.92\left(\mathrm{dt}, J=4.3,13.2 \mathrm{~Hz}, 1 \mathrm{H}\right.$, one of $\left.\mathrm{C} 1-\mathrm{H}_{2}\right), 2.26$ $\left(\mathrm{d}, J=13.4 \mathrm{~Hz}, 1 \mathrm{H}\right.$, one of $\left.\mathrm{C} 3-\mathrm{H}_{2}\right), 2.30\left(\mathrm{dd}, J=4.3,13.0 \mathrm{~Hz}, 1 \mathrm{H}\right.$, one of $\left.\mathrm{C} 7-\mathrm{H}_{2}\right), 2.51(\mathrm{t}, J=13.0 \mathrm{~Hz}, 1 \mathrm{H}$, one of C7- $\left.\mathrm{H}_{2}\right), 2.72(\mathrm{ddq}, J=4.3,13.0,6.4 \mathrm{~Hz}, 1 \mathrm{H}, \mathrm{C} 8-\mathrm{H}), 3.13(\mathrm{~s}, 1 \mathrm{H}, \mathrm{C} 5-\mathrm{H}), 3.75\left(\mathrm{~s}, 3 \mathrm{H}, \mathrm{CO}_{2} \mathrm{CH}_{3}\right), 12.62$ (br s, $\left.1 \mathrm{H}, \mathrm{CO}_{2} \mathrm{H}\right) ;{ }^{13} \mathrm{C}-\mathrm{NMR}\left(125.7 \mathrm{MHz}, \mathrm{CDCl}_{3}\right) \delta 2.8\left(\mathrm{CH}_{3}\right), 17.3\left(\mathrm{CH}_{3}\right), 17.5\left(\mathrm{CH}_{3}\right), 18.4\left(\mathrm{CH}_{2}\right), 28.0$ $\left(\mathrm{CH}_{3}\right), 32.7\left(\mathrm{CH}_{2}\right), 37.0(\mathrm{CH}), 38.7\left(\mathrm{CH}_{2}\right), 43.6(\mathrm{C}), 46.4\left(\mathrm{CH}_{2}\right), 49.6(\mathrm{C}), 52.0\left(\mathrm{CH}_{3}\right), 60.3(\mathrm{CH}), 86.1(\mathrm{C})$, 171.9 (C), 176.0 (C), 218.8 (C); HRMS (ESI) $\mathrm{m} / z$ [M + Na ${ }^{+}$calcd for $\mathrm{C}_{19} \mathrm{H}_{32} \mathrm{O}_{6} \mathrm{SiNa}$ 407.1860; found 407.1847.

Methyl (1S,4R,6R,7R,8S,12R)-1,6,8-trimethyl-2-oxo-7-(trimethylsilyl)oxy-3-oxatricyclo[6.3.1.0 $\left.0^{4,12}\right]$ dodecane -7-carboxylate (23). A solution of $\gamma$-ketocarboxylic acid $27(38.6 \mathrm{mg}, 0.10 \mathrm{mmol})$ in $\mathrm{EtOH}(0.25 \mathrm{~mL}$ plus 2 $\times 0.25 \mathrm{~mL}$ rinse) was added to an ice-cooled $\left(0^{\circ} \mathrm{C}\right)$ solution of $\mathrm{NaBH}_{4}(4.3 \mathrm{mg}, 0.11 \mathrm{mmol})$ in EtOH $(0.5$ $\mathrm{mL})$. After $30 \mathrm{~min}$ of stirring at $0^{\circ} \mathrm{C}, \mathrm{NaBH}_{4}(3.7 \mathrm{mg}, 0.098 \mathrm{mmol})$ was added, and the mixture was stirred at $0{ }^{\circ} \mathrm{C}$ for another $30 \mathrm{~min}$. The reaction was quenched with saturated aqueous $\mathrm{NH}_{4} \mathrm{Cl}(5 \mathrm{~mL})$, and the resulting mixture was partitioned between $\mathrm{AcOEt}(10 \mathrm{~mL})$ and $\mathrm{H}_{2} \mathrm{O}(3 \mathrm{~mL})$. The aqueous layer was extracted with AcOEt $(5 \times 10 \mathrm{~mL})$, and the combined organic extracts were washed with brine $(10 \mathrm{~mL})$, and dried over anhydrous $\mathrm{Na}_{2} \mathrm{SO}_{4}$. Filtration and evaporation in vacuo furnished the crude product $(42.7 \mathrm{mg})$, which was used without further purification.

Ethyl chloroformate $(50 \mu \mathrm{L}, 0.523 \mathrm{mmol})$ was added to an ice-cooled $\left(0^{\circ} \mathrm{C}\right)$ mixture of crude $\gamma$-hydroxycarboxylic acid $(42.7 \mathrm{mg})$ and $\mathrm{Et}_{3} \mathrm{~N}(0.10 \mathrm{~mL}, 0.717 \mathrm{mmol})$ in $\mathrm{CH}_{2} \mathrm{Cl}_{2}(1.7 \mathrm{~mL})$, and the mixture was stirred for $30 \mathrm{~min}$. The reaction was quenched with saturated aqueous $\mathrm{NaHCO}_{3}(5 \mathrm{~mL})$, and the mixture was partitioned between $n$-hexane/AcOEt $(10: 1,22 \mathrm{~mL})$ and $\mathrm{H}_{2} \mathrm{O}(5 \mathrm{~mL})$. The aqueous layer was extracted with $n$-hexane/AcOEt $(10: 1,22 \mathrm{~mL})$, and the combined organic extracts were washed with brine $(20 \mathrm{~mL})$ and dried over anhydrous $\mathrm{Na}_{2} \mathrm{SO}_{4}$. Filtration and evaporation in vacuo followed by column chromatography (silica gel $2.5 \mathrm{~g}$, 9:1 $n$-hexane/AcOEt) afforded lactone 23 (32.6 $\mathrm{mg}, 88 \%$ for two steps) as a colorless oil. $R_{f} 0.44\left(4: 1 n\right.$-hexane/AcOEt); $[\alpha]_{D}^{22}+24.6\left(c 1.07, \mathrm{CHCl}_{3}\right) ; \mathrm{IR}$ (neat) 2953, 1771, 1732, 1456, 1250, 1180, 1142, 1105, 1053, $841 \mathrm{~cm}^{-1} ;{ }^{1} \mathrm{H}-\mathrm{NMR}\left(500 \mathrm{MHz}, \mathrm{CDCl}_{3}\right) \delta 0.15$ $\left(\mathrm{s}, 9 \mathrm{H}, \mathrm{Si}\left(\mathrm{CH}_{3}\right)_{3}\right), 0.79\left(\mathrm{~d}, J=6.4 \mathrm{~Hz}, 3 \mathrm{H}, \mathrm{C} 8-\mathrm{CH}_{3}\right), 0.85\left(\mathrm{dt}, J=13.0,8.5 \mathrm{~Hz}, 1 \mathrm{H}\right.$, one of $\left.\mathrm{C} 1-\mathrm{H}_{2}\right), 1.10(\mathrm{~s}$, $\left.3 \mathrm{H}, \mathrm{C} 10-\mathrm{CH}_{3}\right), 1.30\left(\mathrm{~s}, 3 \mathrm{H}, \mathrm{C} 4-\mathrm{CH}_{3}\right), 1.42-1.56\left(\mathrm{~m}, 3 \mathrm{H}\right.$, one of $\mathrm{C}_{2}-\mathrm{H}_{2}$, one of $\mathrm{C} 3-\mathrm{H}_{2}$, one of $\left.\mathrm{C} 7-\mathrm{H}_{2}\right), 1.69$ $\left(\mathrm{m}, 1 \mathrm{H}\right.$, one of $\left.\mathrm{C} 2-\mathrm{H}_{2}\right), 1.77\left(\mathrm{dd}, J=11.5,13.0 \mathrm{~Hz}, 1 \mathrm{H}\right.$, one of $\left.\mathrm{C} 1-\mathrm{H}_{2}\right), 2.10-2.16\left(\mathrm{~m}, 2 \mathrm{H}\right.$, one of $\mathrm{C} 3-\mathrm{H}_{2}$, C5-H), $2.22\left(\mathrm{dd}, J=6.0,16.2 \mathrm{~Hz}, 1 \mathrm{H}\right.$, one of $\left.\mathrm{C} 7-\mathrm{H}_{2}\right), 2.56(\mathrm{~m}, 1 \mathrm{H}, \mathrm{C} 8-\mathrm{H}), 3.73\left(\mathrm{~s}, 3 \mathrm{H}, \mathrm{CO}_{2} \mathrm{CH}_{3}\right), 4.68$ (br t, $J=5.3 \mathrm{~Hz}, 1 \mathrm{H}, \mathrm{C} 6-H) ;{ }^{13} \mathrm{C}-\mathrm{NMR}\left(125.7 \mathrm{MHz}\right.$, acetone- $\left.d_{6}\right) \delta 2.6\left(\mathrm{CH}_{3}\right), 18.38\left(\mathrm{CH}_{3}\right), 18.40\left(\mathrm{CH}_{2}\right)$, $22.3\left(\mathrm{CH}_{3}\right), 23.4\left(\mathrm{CH}_{3}\right), 29.0\left(\mathrm{CH}_{2}\right), 29.4\left(\mathrm{CH}_{2}\right), 30.6(\mathrm{CH}), 31.9\left(\mathrm{CH}_{2}\right), 40.4(\mathrm{C}), 44.1(\mathrm{C}), 45.6(\mathrm{CH}), 52.0$ $\left(\mathrm{CH}_{3}\right), 76.0(\mathrm{CH}), 86.1(\mathrm{C}), 173.6(\mathrm{C}), 183.2(\mathrm{C})$; HRMS (ESI) $\mathrm{m} / z$ [M $\left.+\mathrm{Na}\right]^{+}$calcd for $\mathrm{C}_{19} \mathrm{H}_{32} \mathrm{O}_{5} \mathrm{SiNa}$ 391.1911; found 391.1917.

Methyl $\quad(1 S, 4 R, 6 R, 7 R, 8 S, 12 R)-7-h y d r o x y-1,6,8$-trimethyl-2-oxo-3-oxatricyclo[6.3.1. $\left.0^{4,12}\right]$ dodecane-7carboxylate (29). $\mathrm{Bu}_{4} \mathrm{NF}$ in THF $(1.0 \mathrm{M}, 2.0 \mathrm{~mL}, 2.0 \mathrm{mmol})$ was added to an ice-cooled $\left(0{ }^{\circ} \mathrm{C}\right)$ solution of TMS ether $23(488 \mathrm{mg}, 1.32 \mathrm{mmol})$ in THF $(14 \mathrm{~mL})$. After $1 \mathrm{~h}$ of stirring at $0{ }^{\circ} \mathrm{C}$, the mixture was 
partitioned between AcOEt $(40 \mathrm{~mL})$ and $\mathrm{H}_{2} \mathrm{O}(15 \mathrm{~mL})$, and the aqueous layer was extracted with AcOEt $(40 \mathrm{~mL})$. The combined organic extracts were washed with brine $(2 \times 20 \mathrm{~mL})$ and dried over anhydrous $\mathrm{Na}_{2} \mathrm{SO}_{4}$. Filtration and evaporation in vacuo furnished the crude product $(634 \mathrm{mg})$, which was purified by column chromatography (silica gel $10 \mathrm{~g}, 10: 1 \rightarrow 3: 1 n$-hexane/AcOEt) to give $\alpha$-hydroxyester 29 (383 mg, 98\%) as a white solid. $R_{f} 0.45$ (2:1 $n$-hexane/AcOEt); mp 142-143 ${ }^{\circ} \mathrm{C}$ (colorless needles from $n$-hexane); $[\alpha]_{D}^{21}+25.0\left(c\right.$ 1.04, $\left.\mathrm{CHCl}_{3}\right) ; \mathrm{IR}(\mathrm{KBr}) 3455,2959,1761,1732,1466$, $1375,1236,1144,1098,1045,932 \mathrm{~cm}^{-1} ;{ }^{1} \mathrm{H}-\mathrm{NMR}\left(500 \mathrm{MHz}, \mathrm{CDCl}_{3}\right) \delta 0.78\left(\mathrm{~d}, J=6.4 \mathrm{~Hz}, 3 \mathrm{H}, \mathrm{C} 8-\mathrm{CH}_{3}\right)$, $0.87\left(\mathrm{dt}, J=13.0,8.5 \mathrm{~Hz}, 1 \mathrm{H}\right.$, one of $\left.\mathrm{C} 1-\mathrm{H}_{2}\right), 1.22\left(\mathrm{~s}, 3 \mathrm{H}, \mathrm{C} 10-\mathrm{CH}_{3}\right), 1.30\left(\mathrm{~s}, 3 \mathrm{H}, \mathrm{C} 4-\mathrm{CH}_{3}\right), 1.44-1.71(\mathrm{~m}$, $5 \mathrm{H}$, one of $\mathrm{C} 1-\mathrm{H}_{2}, \mathrm{C} 2-\mathrm{H}_{2}$, one of $\mathrm{C} 3-\mathrm{H}_{2}$, one of $\left.\mathrm{C} 7-\mathrm{H}_{2}\right), 2.15\left(\mathrm{dt}, J=4.5,13.4 \mathrm{~Hz}, 1 \mathrm{H}\right.$, one of $\left.\mathrm{C} 3-\mathrm{H}_{2}\right), 2.28$ $\left(\mathrm{dd}, J=6.3,16.4 \mathrm{~Hz}, 1 \mathrm{H}\right.$, one of $\left.\mathrm{C} 7-\mathrm{H}_{2}\right), 2.32(\mathrm{~d}, J=4.6 \mathrm{~Hz}, 1 \mathrm{H}, \mathrm{C} 5-\mathrm{H}), 2.53(\mathrm{ddq}, J=6.3,11.7,6.4 \mathrm{~Hz}$, $1 \mathrm{H}, \mathrm{C} 8-\mathrm{H}), 3.33(\mathrm{~s}, 1 \mathrm{H}, \mathrm{C} 9-\mathrm{OH}), 3.82\left(\mathrm{~s}, 3 \mathrm{H}, \mathrm{CO}_{2} \mathrm{CH}_{3}\right), 4.74(\mathrm{dd}, J=4.6,6.8 \mathrm{~Hz}, 1 \mathrm{H}, \mathrm{C} 6-\mathrm{H}) ;{ }^{13} \mathrm{C}-\mathrm{NMR}$ $\left(125.7 \mathrm{MHz}, \mathrm{CDCl}_{3}\right) \delta 16.4\left(\mathrm{CH}_{3}\right), 17.6\left(\mathrm{CH}_{2}\right), 21.6\left(\mathrm{CH}_{3}\right), 23.0\left(\mathrm{CH}_{3}\right), 27.9\left(\mathrm{CH}_{2}\right), 28.1\left(\mathrm{CH}_{2}\right), 29.0(\mathrm{CH})$, $30.7\left(\mathrm{CH}_{2}\right), 38.9(\mathrm{C}), 43.4(\mathrm{C}), 44.7(\mathrm{CH}), 52.8\left(\mathrm{CH}_{3}\right), 75.9(\mathrm{CH}), 80.8(\mathrm{C}), 175.9(\mathrm{C}), 183.6(\mathrm{C})$; HRMS (ESI) $m / z[\mathrm{M}+\mathrm{Na}]^{+}$calcd for $\mathrm{C}_{16} \mathrm{H}_{24} \mathrm{O}_{5} \mathrm{Na} 319.1516$; found 319.1519; Anal. Calcd for $\mathrm{C}_{16} \mathrm{H}_{24} \mathrm{O}_{5}: \mathrm{C}, 64.84 ; \mathrm{H}$, 8.16. Found: $\mathrm{C}, 64.82 ; \mathrm{H}, 8.06$.

$\left(2^{\prime} R, 2 a S, 5 a S, 6 R, 7 R, 8 a R\right)-2 a, 5 a, 7-T r i m e t h y l o c t a h y d r o s p i r o[6 H$-naphtho[1,8-bc]furan-6,2'-oxiran]-2(2aH)-one (22). Sodium bis(2-methoxyethoxy)aluminum hydride in toluene $(3.3 \mathrm{M}, 1.1 \mathrm{~mL}, 3.6 \mathrm{mmol}$ ) was diluted with $\mathrm{CH}_{2} \mathrm{Cl}_{2}(5.6 \mathrm{~mL})$, and the solution was cooled to $-78^{\circ} \mathrm{C}$. A solution of $\alpha$-hydroxyester 29 ( $255 \mathrm{mg}, 0.86 \mathrm{mmol})$ in $\mathrm{CH}_{2} \mathrm{Cl}_{2}(2 \mathrm{~mL}$ plus $2 \times 0.5 \mathrm{~mL}$ rinse) was added, and the mixture was stirred at $-23{ }^{\circ} \mathrm{C}$ for $22 \mathrm{~h}$. The reaction was quenched by addition of $\mathrm{MeOH}(3 \mathrm{~mL})$ followed by $10 \%$ aqueous potassium sodium tartrate $(12 \mathrm{~mL})$. After $3 \mathrm{~h}$ of stirring, the resulting mixture was extracted with $\operatorname{AcOEt}(2 \times 40 \mathrm{~mL})$, and the combined organic extracts were washed with brine $(2 \times 20 \mathrm{~mL})$ and dried over anhydrous $\mathrm{Na}_{2} \mathrm{SO}_{4}$. Filtration and evaporation in vacuo furnished the crude product $(235 \mathrm{mg})$, which was used without further purification.

This sequence was repeated employing bis(2-methoxyethoxy)aluminum hydride in toluene ( $3.3 \mathrm{M}$, $0.16 \mathrm{~mL}, 0.53 \mathrm{mmol})$ and $\mathrm{CH}_{2} \mathrm{Cl}_{2}(4 \mathrm{~mL})$ with the reaction time of $16 \mathrm{~h}$ at $-23^{\circ} \mathrm{C}$. The crude product $(216 \mathrm{mg})$ was used without further purification.

A solution of crude diol $31(216 \mathrm{mg})$ in THF ( $2 \mathrm{~mL}$ plus $1 \mathrm{~mL}$ and $2 \times 0.5 \mathrm{~mL}$ rinse) was added dropwise to an ice-cooled $\left(0{ }^{\circ} \mathrm{C}\right)$ suspension of $\mathrm{NaH}(60 \%$ in oil, $240 \mathrm{mg}, 6.01 \mathrm{mmol})$ in THF $(4.6 \mathrm{~mL})$. After $30 \mathrm{~min}$ of stirring at $0{ }^{\circ} \mathrm{C}, p$-toluenesulfonyl imidazole $(577 \mathrm{mg}, 2.59 \mathrm{mmol})$ was added, and the mixture was stirred at $0{ }^{\circ} \mathrm{C}$ for $11 \mathrm{~h}$. The reaction was quenched with saturated aqueous $\mathrm{NH}_{4} \mathrm{Cl}(10$ $\mathrm{mL})$, and the resulting mixture was extracted with AcOEt $(2 \times 40 \mathrm{~mL})$. The combined organic extracts were washed with brine $(2 \times 20 \mathrm{~mL})$, and dried over anhydrous $\mathrm{Na}_{2} \mathrm{SO}_{4}$. Filtration and evaporation in vacuo furnished the crude product $(563 \mathrm{mg}$ ), which was purified by column chromatography (silica gel $15 \mathrm{~g}, 40: 1 \rightarrow$ 10:1 $n$-hexane/AcOEt) to give epoxide 22 (182 $\mathrm{mg}, 84 \%$ for three steps) as a white solid. $R_{f} 0.53$ (2:1 $n$-hexane/AcOEt); mp 83-84 ${ }^{\circ} \mathrm{C}$ (colorless needles from 8:1 $n$-hexane/Et $\left.{ }_{2} \mathrm{O}\right) ;[\alpha]_{D}^{21}+13.7(c$ 1.21, $\left.\mathrm{CHCl}_{3}\right) ; \mathrm{IR}(\mathrm{KBr}) 2941,1761,1464,1393,1354,1209,1105,997 \mathrm{~cm}^{-1} ;{ }^{1} \mathrm{H}-\mathrm{NMR}\left(500 \mathrm{MHz}, \mathrm{CDCl}_{3}\right) \delta$ $0.76\left(\mathrm{~d}, J=6.6 \mathrm{~Hz}, 3 \mathrm{H}, \mathrm{C} 8-\mathrm{CH}_{3}\right), 0.97\left(\mathrm{dt}, J=12.4,8.5 \mathrm{~Hz}, 1 \mathrm{H}\right.$, one of $\left.\mathrm{C} 1-\mathrm{H}_{2}\right), 1.24\left(\mathrm{~s}, 3 \mathrm{H}, \mathrm{C} 10-\mathrm{CH}_{3}\right), 1.30$ $\left(\mathrm{s}, 3 \mathrm{H}, \mathrm{C} 4-\mathrm{CH}_{3}\right), 1.35\left(\mathrm{dd}, J=10.1,12.4 \mathrm{~Hz}, 1 \mathrm{H}\right.$, one of $\left.\mathrm{C} 1-\mathrm{H}_{2}\right), 1.45-1.51\left(\mathrm{~m}, 2 \mathrm{H}\right.$, one of $\mathrm{C} 2-\mathrm{H}_{2}$, one of C3- $\left.H_{2}\right), 1.66\left(\mathrm{ddd}, J=4.7,11.8,16.0 \mathrm{~Hz}, 1 \mathrm{H}\right.$, one of $\left.\mathrm{C} 7-\mathrm{H}_{2}\right), 1.72\left(\mathrm{~m}, 1 \mathrm{H}\right.$, one of $\left.\mathrm{C} 2-\mathrm{H}_{2}\right), 1.90(\mathrm{~d}, J=4.7$ $\mathrm{Hz}, 1 \mathrm{H}, \mathrm{C} 5-\mathrm{H}), 2.14\left(\mathrm{dt}, J=5.4,15.5 \mathrm{~Hz}, 1 \mathrm{H}\right.$, one of $\left.\mathrm{C} 3-\mathrm{H}_{2}\right), 2.37\left(\mathrm{dd}, J=6.1,16.0 \mathrm{~Hz}, 1 \mathrm{H}\right.$, one of C7- $\left.\mathrm{H}_{2}\right)$, $2.55(\mathrm{ddq}, J=6.1,11.8,6.6 \mathrm{~Hz}, 1 \mathrm{H}, \mathrm{C} 8-\mathrm{H}), 2.63\left(\mathrm{~d}, J=3.9 \mathrm{~Hz}, 1 \mathrm{H}\right.$, one of $\left.\mathrm{C} 11-\mathrm{H}_{2}\right), 2.71(\mathrm{~d}, J=3.9 \mathrm{~Hz}$, $1 \mathrm{H}$, one of $\left.\mathrm{C} 11-\mathrm{H}_{2}\right), 4.79(\mathrm{br} \mathrm{t}, J=4.7 \mathrm{~Hz}, 1 \mathrm{H}, \mathrm{C} 6-\mathrm{H}) ;{ }^{13} \mathrm{C}-\mathrm{NMR}\left(125.7 \mathrm{MHz}, \mathrm{CDCl}_{3}\right) \delta 15.0\left(\mathrm{CH}_{3}\right), 17.3$ $\left(\mathrm{CH}_{2}\right), 22.4\left(\mathrm{CH}_{3}\right), 24.9(\mathrm{CH}), 25.0\left(\mathrm{CH}_{3}\right), 26.8\left(\mathrm{CH}_{2}\right), 28.1(\mathrm{C}), 32.5\left(\mathrm{CH}_{2}\right), 34.6(\mathrm{C}), 44.1(\mathrm{C}), 45.9\left(\mathrm{CH}_{2}\right)$, $47.5(\mathrm{CH}), 63.7(\mathrm{C}), 76.0(\mathrm{CH}), 183.3(\mathrm{C})$; HRMS (ESI) $\mathrm{m} / z[\mathrm{M}+\mathrm{Na}]^{+}$calcd for $\mathrm{C}_{15} \mathrm{H}_{22} \mathrm{O}_{3} \mathrm{Na} 273.1461$; found 273.1469 .

Marrubiin (1). To a cooled $\left(-10^{\circ} \mathrm{C}\right)$ suspension of $\mathrm{CuBr} \cdot \mathrm{SMe}_{2}(65.1 \mathrm{mg}, 0.316 \mathrm{mmol})$ in $\mathrm{Et}_{2} \mathrm{O}(1.5 \mathrm{~mL})$ was added a $0.14 \mathrm{M}$ solution of (3-furylmethyl)magnesium bromide (32) in $\mathrm{Et}_{2} \mathrm{O}$ (3.35 mL, $0.69 \mathrm{mmol}$ ) [prepared from 3-(bromomethyl)furan (563 mg, $3.37 \mathrm{mmol}$ ) and magnesium (103 mg, $4.22 \mathrm{mmol}$ ) in 
$\mathrm{Et}_{2} \mathrm{O}(4 \mathrm{~mL})$ at $\left.0{ }^{\circ} \mathrm{C}\right]$, followed by addition of a solution of epoxide $22(40.2 \mathrm{mg}, 0.161 \mathrm{mmol})$ in $\mathrm{Et}_{2} \mathrm{O}$ $\left(0.5 \mathrm{~mL}\right.$ plus $2 \times 0.5 \mathrm{~mL}$ rinse). After $2 \mathrm{~h}$, the reaction was quenched with saturated aqueous $\mathrm{NH}_{4} \mathrm{Cl}(5$ $\mathrm{mL})$, and the resulting mixture was extracted with AcOEt $(3 \times 10 \mathrm{~mL})$. The combined organic extracts were washed with brine $(20 \mathrm{~mL})$, and dried over anhydrous $\mathrm{Na}_{2} \mathrm{SO}_{4}$. Filtration and evaporation in vacuo furnished the crude product $(200 \mathrm{mg}$ ), which was chromatographed twice (silica gel $5 \mathrm{~g}, 20: 1 \rightarrow$ 10:1 $n$-hexane/AcOEt) to give marrubiin $(\mathbf{1}, 34.7 \mathrm{mg}, 65 \%)$ and isomer $33(4.7 \mathrm{mg}, 9 \%)$ as white solids. $R_{f} 0.59$ (1:1 $n$-hexane/AcOEt); mp $160-161{ }^{\circ} \mathrm{C}$ (colorless needles from 4:1 $n$-hexane/AcOEt) (lit. [38], mp $\left.160{ }^{\circ} \mathrm{C}\right) ;[\alpha]_{D}^{21}+34.4$ (c 1.04, $\mathrm{CHCl}_{3}$ ) [lit. [38], [ $\left.\alpha\right]_{D}^{20}+35.8$ (c 3.1, $\left.\mathrm{CHCl}_{3}\right)$ ]; IR (KBr) 3466, 2940, 2870, 1740, 1468, 1395, 1356, 1304, 1269, 1200, 1153, 1101, 1024, $984 \mathrm{~cm}^{-1} ;{ }^{1} \mathrm{H}-\mathrm{NMR}\left(500 \mathrm{MHz}, \mathrm{CDCl}_{3}\right) \delta 0.97(\mathrm{~d}, J=$ $\left.6.4 \mathrm{~Hz}, 3 \mathrm{H}, \mathrm{C} 8-\mathrm{CH}_{3}\right), 1.07\left(\mathrm{~s}, 3 \mathrm{H}, \mathrm{C} 10-\mathrm{CH}_{3}\right), 1.26(\mathrm{~s}, 1 \mathrm{H}, \mathrm{C} 9-\mathrm{OH}), 1.29\left(\mathrm{~s}, 3 \mathrm{H}, \mathrm{C} 4-\mathrm{CH}_{3}\right), 1.32(\mathrm{dt}, J=12.8$, $8.5 \mathrm{~Hz}, 1 \mathrm{H}$, one of $\left.\mathrm{C} 1-\mathrm{H}_{2}\right), 1.43-1.55\left(\mathrm{~m}, 2 \mathrm{H}\right.$, one of $\mathrm{C} 2-\mathrm{H}_{2}$, one of $\left.\mathrm{C} 3-\mathrm{H}_{2}\right), 1.66-1.79(\mathrm{~m}, 4 \mathrm{H}$, one of $\mathrm{C} 1-\mathrm{H}_{2}$, one of $\mathrm{C} 2-\mathrm{H}_{2}$, one of $\mathrm{C} 7-\mathrm{H}_{2}$, one of $\left.\mathrm{C} 11-\mathrm{H}_{2}\right), 1.90\left(\mathrm{ddd}, J=7.2,10.1,14.4 \mathrm{~Hz}, 1 \mathrm{H}\right.$, one of $\left.\mathrm{C} 11-\mathrm{H}_{2}\right)$, 2.09-2.18 (m, 3H, one of C3- $\mathrm{H}_{2}$, one of $\left.\mathrm{C} 7-\mathrm{H}_{2}, \mathrm{C} 8-\mathrm{H}\right), 2.23(\mathrm{~d}, \mathrm{~J}=4.6 \mathrm{~Hz}, 1 \mathrm{H}, \mathrm{C} 5-\mathrm{H}), 2.48-2.58(\mathrm{~m}, 2 \mathrm{H}$, C12- $\left.\mathrm{H}_{2}\right), 4.74$ (br dd, J = 4.6, $\left.6.5 \mathrm{~Hz}, 1 \mathrm{H}, \mathrm{C} 6-\mathrm{H}\right), 6.27$ (s, 1H, C14-H), $7.24(\mathrm{~s}, 1 \mathrm{H}, \mathrm{C} 16-\mathrm{H}), 7.37$ (s, 1H, C15-H); ${ }^{13} \mathrm{C}-\mathrm{NMR}\left(125.7 \mathrm{MHz}, \mathrm{CDCl}_{3}\right) \delta 16.6\left(\mathrm{CH}_{3}\right), 18.2\left(\mathrm{CH}_{2}\right), 21.0\left(\mathrm{CH}_{2}\right), 22.3\left(\mathrm{CH}_{3}\right), 22.9\left(\mathrm{CH}_{3}\right)$, $28.3\left(\mathrm{CH}_{2}\right), 28.6\left(\mathrm{CH}_{2}\right), 31.5\left(\mathrm{CH}_{2}\right), 32.4(\mathrm{CH}), 35.1\left(\mathrm{CH}_{2}\right), 39.7(\mathrm{C}), 43.8(\mathrm{C}), 44.8(\mathrm{CH}), 75.8(\mathrm{C}), 76.2$ $(\mathrm{CH}), 110.7(\mathrm{CH}), 125.0(\mathrm{C}), 138.6(\mathrm{CH}), 143.1(\mathrm{CH}), 183.8(\mathrm{C})$; HRMS (ESI) $\mathrm{m} / \mathrm{z}[\mathrm{M}+\mathrm{Na}]^{+}$calcd for $\mathrm{C}_{20} \mathrm{H}_{28} \mathrm{O}_{4} \mathrm{Na}$ 355.1880; found 355.1867.

Data for $(1 S, 4 R, 6 R, 7 R, 8 S, 12 R)-7-[(3-m e t h y l f u r a n-2-y l) m e t h y l]-7-h y d r o x y-1,6,8-t r i m e t h y l-2-o x o-3-$ oxatricyclo[6.3.1.0 $0^{4,12}$ ]dodecane (33): $R_{f} 0.27$ (3:1 $n$-hexane/AcOEt); mp 173-174 ${ }^{\circ} \mathrm{C}$ (colorless needles from 3:1 n-hexane/AcOEt); $[\alpha]_{D}^{22}+12.0\left(c 0.38, \mathrm{CHCl}_{3}\right)$; IR (KBr) 3453, 2955, 2928, 2874, 1738, 1456, 1393, 1352, 1300, 1279, 1198, 1152, 1138, 999, $982 \mathrm{~cm}^{-1} ;{ }^{1} \mathrm{H}-\mathrm{NMR}\left(500 \mathrm{MHz}, \mathrm{CDCl}_{3}\right) \delta 0.85(\mathrm{~d}, J=6.4 \mathrm{~Hz}, 3 \mathrm{H}$, $\left.\mathrm{C} 8-\mathrm{CH}_{3}\right), 1.07\left(\mathrm{~s}, 3 \mathrm{H}, \mathrm{C} 10-\mathrm{CH}_{3}\right), 1.15\left(\mathrm{~m}, 1 \mathrm{H}\right.$, one of $\left.\mathrm{C} 1-\mathrm{H}_{2}\right), 1.28\left(\mathrm{~s}, 3 \mathrm{H}, \mathrm{C} 4-\mathrm{CH}_{3}\right), 1.41-1.52(\mathrm{~m}, 2 \mathrm{H}$, one of $\mathrm{C} 1-\mathrm{H}_{2}$, one of $\left.\mathrm{C} 2-\mathrm{H}_{2}\right), 1.66-1.74\left(\mathrm{~m}, 3 \mathrm{H}\right.$, one of $\mathrm{C} 2-\mathrm{H}_{2}$, one of $\mathrm{C} 3-\mathrm{H}_{2}$, one of $\left.\mathrm{C} 7-\mathrm{H}_{2}\right), 1.98(\mathrm{~s}, 3 \mathrm{H}$, $\left.\mathrm{C} 13-\mathrm{CH}_{3}\right), 2.06-2.21\left(\mathrm{~m}, 3 \mathrm{H}\right.$, one of $\mathrm{C} 3-\mathrm{H}_{2}$, one of $\left.\mathrm{C} 7-\mathrm{H}_{2}, \mathrm{C} 8-\mathrm{H}\right), 2.28(\mathrm{~d}, J=4.7 \mathrm{~Hz}, 1 \mathrm{H}, \mathrm{C} 5-\mathrm{H}), 2.33$ (s, $1 \mathrm{H}, \mathrm{C} 9-\mathrm{OH}), 2.78\left(\mathrm{~d}, J=15.5 \mathrm{~Hz}, 1 \mathrm{H}\right.$, one of C11- $\left.\mathrm{H}_{2}\right), 2.88\left(\mathrm{~d}, J=15.5 \mathrm{~Hz}, 1 \mathrm{H}\right.$, one of C11- $\left.\mathrm{H}_{2}\right), 4.73(\mathrm{ddd}$, $J=1.4,4.7,6.1 \mathrm{~Hz}, 1 \mathrm{H}, \mathrm{C} 6-H), 6.19(\mathrm{~d}, J=1.8 \mathrm{~Hz}, 1 \mathrm{H}, \mathrm{C} 14-H), 7.28(\mathrm{~d}, J=1.8 \mathrm{~Hz}, 1 \mathrm{H}, \mathrm{C} 15-H) ;{ }^{13} \mathrm{C}-\mathrm{NMR}$ $\left(125.7 \mathrm{MHz}, \mathrm{CDCl}_{3}\right) \delta 10.3\left(\mathrm{CH}_{3}\right), 16.3\left(\mathrm{CH}_{3}\right), 18.3\left(\mathrm{CH}_{2}\right), 22.2\left(\mathrm{CH}_{3}\right), 22.9\left(\mathrm{CH}_{3}\right), 28.23\left(\mathrm{CH}_{2}\right), 28.24$ $\left(\mathrm{CH}_{2}\right), 30.6\left(\mathrm{CH}_{2}\right), 31.93(\mathrm{CH}), 31.94\left(\mathrm{CH}_{2}\right), 39.6(\mathrm{C}), 43.8(\mathrm{C}), 44.6(\mathrm{CH}), 76.2(\mathrm{CH}), 76.9(\mathrm{C}), 113.3(\mathrm{CH})$, 116.4 (C), $140.5(\mathrm{CH}), 148.0(\mathrm{C}), 183.9$ (C); HRMS (ESI) $\mathrm{m} / z$ [M + Na] ${ }^{+}$calcd for $\mathrm{C}_{20} \mathrm{H}_{28} \mathrm{O}_{4} \mathrm{Na} 355.1880$; found 355.1871 .

(1S,4R,6R,7R,8S,12R)-7-[2-[2-(Trimethylsilyl)furan-3-yl]ethyl]]-7-hydroxy-1,6,8-trimethyl-2-oxo-3-oxatricyclo [6.3.1. $0^{4,12}$ ]dodecane (35). Phosphorus tribromide $(0.28 \mathrm{~mL}, 2.98 \mathrm{mmol})$ was added to an ice-cooled $\left(0{ }^{\circ} \mathrm{C}\right)$ solution of [2-(trimethylsilyl)furan-3-yl]methanol $(998 \mathrm{mg}, 5.86 \mathrm{mmol}) \mathrm{in}_{\mathrm{Et}} \mathrm{O}(30 \mathrm{~mL})$. After $30 \mathrm{~min}$ of stirring, the reaction was quenched with brine $(30 \mathrm{~mL})$, and the resulting mixture was extracted with $\mathrm{Et}_{2} \mathrm{O}(30 \mathrm{~mL})$. The organic extract was dried over anhydrous $\mathrm{Na}_{2} \mathrm{SO}_{4}$. Filtration and evaporation in vacuo furnished the pale brown oil, which was purified by distillation to give 3-(bromomethyl)-2-(trimethylsilyl)furan $(618 \mathrm{mg}$, $45 \%)$ as a pale brown oil.

A solution of 3-(bromomethyl)-2-(trimethylsilyl)furan $(584 \mathrm{mg}, 2.50 \mathrm{mmol})$ in $\mathrm{Et}_{2} \mathrm{O}(1$ $\mathrm{mL})$ was added to a cooled $\left(-10^{\circ} \mathrm{C}\right)$ suspension of magnesium tuning (102 $\left.\mathrm{mg}, 4.20 \mathrm{mmol}\right)$ in $\mathrm{Et}_{2} \mathrm{O}(1 \mathrm{~mL})$, and the reaction mixture was stirred for $1.5 \mathrm{~h}$. The $0.23 \mathrm{M}$ solution of [2-(trimethylsilyl)furan-3-yl]methylmagnesium bromide (34) in $\mathrm{Et}_{2} \mathrm{O}(1.5 \mathrm{~mL}, 0.345 \mathrm{mmol}$ ) thus obtained was added to a cooled $\left(-10^{\circ} \mathrm{C}\right)$ suspension of $\mathrm{CuBr} \cdot \mathrm{SMe}_{2}(69.6 \mathrm{mg}, 0.339 \mathrm{mmol})$ in $\mathrm{Et}_{2} \mathrm{O}(0.6$ $\mathrm{mL})$, followed by addition of a solution of epoxide $22(42.6 \mathrm{mg}, 0.170 \mathrm{mmol})$ in $\mathrm{Et}_{2} \mathrm{O}(1.0 \mathrm{~mL})$. After $4 \mathrm{~h}$ of stirring, the reaction was quenched with saturated aqueous $\mathrm{NH}_{4} \mathrm{Cl}(3 \mathrm{~mL})$, and the resulting mixture was extracted with AcOEt $(3 \times 10 \mathrm{~mL})$. The combined organic extracts were washed with brine $(10$ $\mathrm{mL}$ ), and dried over anhydrous $\mathrm{Na}_{2} \mathrm{SO}_{4}$. Filtration and evaporation in vacuo furnished the pale brown oil (206 mg), which was purified by flash column chromatography (silica gel $4 \mathrm{~g}$, $n$-hexane $\rightarrow$ 40:1 $\rightarrow$ 20:1 $\rightarrow$ 5:1 $n$-hexane/AcOEt) to give silylated marrubiin $35(52.0 \mathrm{mg}, 76 \%)$ as a colorless solid. $R_{f} 0.32$ (3:1 n-hexane/AcOEt); $[\alpha]_{D}^{24}+29.3$ (c 2.05, acetone); IR (neat) 3479, 3417, 2954, 2870, 1749, 1633, 1568, 
1464, 1387, 1248, 1197, 1149, 1089, 1045, 1020, 991, 914, $840 \mathrm{~cm}^{-1} ;{ }^{1} \mathrm{H}-\mathrm{NMR}\left(500 \mathrm{MHz}, \mathrm{CDCl}_{3}\right) \delta 0.29$ (s, 9H, $\left.\mathrm{Si}\left(\mathrm{CH}_{3}\right)_{3}\right), 0.99\left(\mathrm{~d}, \mathrm{~J}=6.5 \mathrm{~Hz}, 3 \mathrm{H}, \mathrm{C} 8-\mathrm{CH}_{3}\right), 1.06\left(\mathrm{~s}, 3 \mathrm{H}, \mathrm{C} 10-\mathrm{CH}_{3}\right), 1.29\left(\mathrm{~s}, 3 \mathrm{H}, \mathrm{C} 4-\mathrm{CH}_{3}\right), 1.30(\mathrm{~m}$, $1 \mathrm{H}$, one of $\left.\mathrm{C} 1-\mathrm{H}_{2}\right), 1.45\left(\mathrm{~m}, 1 \mathrm{H}\right.$, one of $\left.\mathrm{C} 3-\mathrm{H}_{2}\right), 1.52\left(\mathrm{~m}, 1 \mathrm{H}\right.$, one of $\left.\mathrm{C} 2-\mathrm{H}_{2}\right), 1.68-1.75(\mathrm{~m}, 4 \mathrm{H}$, one of C1- $\mathrm{H}_{2}$, one of $\mathrm{C} 2-\mathrm{H}_{2}$, one of $\mathrm{C} 7-\mathrm{H}_{2}$, one of $\left.\mathrm{C} 11-\mathrm{H}_{2}\right), 1.87\left(\mathrm{ddd}, \mathrm{J}=7.1,10.3,14.5 \mathrm{~Hz}, 1 \mathrm{H}\right.$, one of $\left.\mathrm{C} 11-\mathrm{H}_{2}\right)$, 2.09-2.19 (m, 3H, one of C3- $\mathrm{H}_{2}$, one of $\left.\mathrm{C} 7-\mathrm{H}_{2}, \mathrm{C} 8-\mathrm{H}\right), 2.23(\mathrm{~d}, \mathrm{~J}=4.6 \mathrm{~Hz}, 1 \mathrm{H}, \mathrm{C} 5-\mathrm{H}), 2.56-2.75(\mathrm{~m}, 2 \mathrm{H}$, $\left.\mathrm{C} 12-\mathrm{H}_{2}\right), 4.74(\mathrm{dd}, J=4.6,6.3 \mathrm{~Hz}, 1 \mathrm{H}, \mathrm{C} 6-\mathrm{H}), 6.27(\mathrm{~d}, J=1.2 \mathrm{~Hz}, 1 \mathrm{H}, \mathrm{C} 14-H), 7.55(\mathrm{~d}, J=1.2 \mathrm{~Hz}, 1 \mathrm{H}$, C15- $H) ;{ }^{13} \mathrm{C}-\mathrm{NMR}\left(125.7 \mathrm{MHz}, \mathrm{CDCl}_{3}\right) \delta-0.9\left(\mathrm{CH}_{3}\right), 16.6\left(\mathrm{CH}_{3}\right), 18.2\left(\mathrm{CH}_{2}\right), 21.7\left(\mathrm{CH}_{2}\right), 22.3\left(\mathrm{CH}_{3}\right)$, $22.9\left(\mathrm{CH}_{3}\right), 28.3\left(\mathrm{CH}_{2}\right), 28.7\left(\mathrm{CH}_{2}\right), 31.5\left(\mathrm{CH}_{2}\right), 32.4(\mathrm{CH}), 36.2\left(\mathrm{CH}_{2}\right), 39.7(\mathrm{C}), 43.8(\mathrm{C}), 44.9(\mathrm{CH}), 75.8$ (C), $76.2(\mathrm{CH}), 110.7(\mathrm{CH}), 135.0(\mathrm{C}), 146.4(\mathrm{CH}), 154.3(\mathrm{C}), 183.9(\mathrm{C})$; HRMS (ESI) $\mathrm{m} / z$ [M + Na] ${ }^{+}$calcd for $\mathrm{C}_{23} \mathrm{H}_{36} \mathrm{O}_{4} \mathrm{SiNa}$ 427.2275; found 427.2259.

Marrubiin (1). $\mathrm{Bu}_{4} \mathrm{NF}$ in THF $(1.0 \mathrm{M}, 20 \mu \mathrm{L}, 20 \mu \mathrm{mol})$ was added to a solution of silylated marrubiin 35 $(0.7 \mathrm{mg}, 1.7 \mu \mathrm{mol})$ in THF $(0.2 \mathrm{~mL})$. After $4 \mathrm{~h}$ of stirring, the mixture was partitioned between AcOEt $(8$ $\mathrm{mL})$ and $\mathrm{H}_{2} \mathrm{O}(8 \mathrm{~mL})$, and the aqueous layer was extracted with AcOEt $(8 \mathrm{~mL})$. The combined organic extracts were washed with brine $(8 \mathrm{~mL})$ and dried over anhydrous $\mathrm{Na}_{2} \mathrm{SO}_{4}$. Filtration and evaporation in vacuo furnished the colorless oil $(0.5 \mathrm{mg})$, which was purified by column chromatography (silica gel $1 \mathrm{~g}, 4: 1 \rightarrow 1: 1 \mathrm{n}$-hexane/AcOEt) to give marrubiin $(\mathbf{1}, 0.4 \mathrm{mg}, 70 \%)$ as a white solid.

(1S,4R,6R,7R,8S,12R)-7-[2-(2,5-Diethoxy-2,5-dihydrofuran-3-yl)ethyl]-7-hydroxy-1,6,8-trimethyl-2-oxo-3-oxa tricyclo[6.3.1.0 $\left.{ }^{4,12}\right]$ dodecane (36). Pyridinium tribromide $(11.0 \mathrm{mg}, 34 \mu \mathrm{mol})$ was added to an ice-cooled $\left(0{ }^{\circ} \mathrm{C}\right)$ solution of marrubiin $(1,10.4 \mathrm{mg}, 31 \mu \mathrm{mol})$ in $\mathrm{CH}_{2} \mathrm{Cl}_{2} / \mathrm{EtOH}(1: 1,0.6 \mathrm{~mL})$. After $10 \mathrm{~min}$ of stirring, the reaction was quenched with a mixture of saturated aqueous $\mathrm{NaHCO}_{3}(3 \mathrm{~mL})$ and $1 \mathrm{M}$ aqueous $\mathrm{Na}_{2} \mathrm{~S}_{2} \mathrm{O}_{3}(2 \mathrm{~mL})$, and the resulting mixture was extracted with AcOEt $(2 \times 20 \mathrm{~mL})$. The combined organic extracts were washed with brine $(30 \mathrm{~mL})$, and dried over anhydrous $\mathrm{Na}_{2} \mathrm{SO}_{4}$. Filtration and evaporation in vacuo furnished the crude product $(14.7 \mathrm{mg})$, which was purified by column chromatography (silica gel $2.5 \mathrm{~g}, 3: 1 \rightarrow 1: 1 n$-hexane/AcOEt) to give bisacetals $36(10.4 \mathrm{mg}$, $79 \%, \mathrm{dr}=2: 2: 1: 1)$ as a colorless oil. $R_{f} 0.58\left(2: 3 n\right.$-hexane/AcOEt); $[\alpha]_{D}^{23}+33.9\left(c 1.19, \mathrm{CHCl}_{3}\right) ; \mathrm{IR}$ (neat) 3516, 2972, 2930, 1767, 1755, 1458, 1373, 1346, 1198, 1101, 1045, 1018, $984 \mathrm{~cm}^{-1}$; ${ }^{1} \mathrm{H}-\mathrm{NMR}(500 \mathrm{MHz}$, $\left.\mathrm{CDCl}_{3}\right) \delta 0.92\left(\mathrm{~d}, J=6.3 \mathrm{~Hz}, 3 \mathrm{H}, \mathrm{C} 8-\mathrm{CH}_{3}\right), 1.05\left(\mathrm{~s}, 3 \mathrm{H}, \mathrm{C} 10-\mathrm{CH}_{3}\right), 1.21-1.34(\mathrm{~m}, 11 \mathrm{H}), 1.43-1.56(\mathrm{~m}, 2 \mathrm{H})$, 1.66-1.77 $(\mathrm{m}, 4 \mathrm{H}), 1.86\left(\mathrm{~m}, 1 \mathrm{H}\right.$, one of $\left.\mathrm{CH}_{2}\right), 2.05-2.32(\mathrm{~m}, 6 \mathrm{H}), 3.53-3.82\left(\mathrm{~m}, 4 \mathrm{H}, 2 \times \mathrm{OCH}_{2} \mathrm{CH}_{3}\right), 4.74$ $(\mathrm{m}, 1 \mathrm{H}, \mathrm{C} 6-H), 5.48(\mathrm{~s}, 0.65 \mathrm{H}, \mathrm{C} 15-H), 5.60(\mathrm{~s}, 0.65 \mathrm{H}, \mathrm{C} 16-H), 5.67(\mathrm{~s}, 1 \mathrm{H}, \mathrm{C} 14-H), 5.75$ (br d, J = 3.6 $\mathrm{Hz}, 0.35 \mathrm{H}, \mathrm{C} 16-H), 5.85$ (br d, $J=3.6 \mathrm{~Hz}, 0.35 \mathrm{H}, \mathrm{C} 15-\mathrm{H}) ;{ }^{13} \mathrm{C}-\mathrm{NMR}\left(125.7 \mathrm{MHz}, \mathrm{CDCl}_{3}\right) \delta 15.3\left(\mathrm{CH}_{3}\right)$, $15.4\left(\mathrm{CH}_{3}\right), 16.50\left(\mathrm{CH}_{3}\right), 16.51\left(\mathrm{CH}_{3}\right), 16.54\left(\mathrm{CH}_{3}\right), 18.09\left(\mathrm{CH}_{2}\right), 18.12\left(\mathrm{CH}_{2}\right), 22.22\left(\mathrm{CH}_{3}\right), 22.23\left(\mathrm{CH}_{3}\right)$, 22.26 $\left(\mathrm{CH}_{3}\right), 22.28\left(\mathrm{CH}_{3}\right), 22.31\left(\mathrm{CH}_{2}\right), 22.4\left(\mathrm{CH}_{2}\right), 22.49\left(\mathrm{CH}_{2}\right), 22.52\left(\mathrm{CH}_{2}\right), 22.87\left(\mathrm{CH}_{3}\right), 22.89\left(\mathrm{CH}_{3}\right)$, $22.96\left(\mathrm{CH}_{3}\right), 22.97\left(\mathrm{CH}_{3}\right), 28.27\left(\mathrm{CH}_{2}\right), 28.28\left(\mathrm{CH}_{2}\right), 28.59\left(\mathrm{CH}_{2}\right), 28.61\left(\mathrm{CH}_{2}\right), 28.63\left(\mathrm{CH}_{2}\right), 31.4\left(\mathrm{CH}_{2}\right)$, $31.46\left(\mathrm{CH}_{2}\right), 31.47\left(\mathrm{CH}_{2}\right), 32.0\left(\mathrm{CH}_{2}\right), 32.11\left(\mathrm{CH}_{2}\right), 32.15(\mathrm{CH}), 32.22(\mathrm{CH}), 32.29\left(\mathrm{CH}_{2}\right), 32.33\left(\mathrm{CH}_{2}\right)$, $32.5(\mathrm{CH}), 39.69(\mathrm{C}), 39.70(\mathrm{C}), 39.71(\mathrm{C}), 39.73(\mathrm{C}), 43.71(\mathrm{C}), 43.72(\mathrm{C}), 43.76(\mathrm{C}), 44.7(\mathrm{CH}), 44.8(\mathrm{CH})$, $62.3\left(\mathrm{CH}_{2}\right), 62.61\left(\mathrm{CH}_{2}\right), 62.62\left(\mathrm{CH}_{2}\right), 62.8\left(\mathrm{CH}_{2}\right), 63.1\left(\mathrm{CH}_{2}\right), 63.41\left(\mathrm{CH}_{2}\right), 63.42\left(\mathrm{CH}_{2}\right), 63.5\left(\mathrm{CH}_{2}\right)$, $75.37(\mathrm{C}), 75.388(\mathrm{C}), 75.394(\mathrm{C}), 76.06(\mathrm{CH}), 76.12(\mathrm{CH}), 76.13(\mathrm{CH}), 105.95(\mathrm{CH}), 105.97(\mathrm{CH}), 106.937$ $(\mathrm{CH}), 106.943(\mathrm{CH}), 107.5(\mathrm{CH}), 107.6(\mathrm{CH}), 108.2(\mathrm{CH}), 108.3(\mathrm{CH}), 123.8(\mathrm{CH}), 124.0(\mathrm{CH}), 124.1(\mathrm{CH})$, $124.3(\mathrm{CH}), 145.3(\mathrm{C}), 145.78$ (C), 145.81 (C), 183.7 (C), 183.8 (C); HRMS (ESI) $\mathrm{m} / z$ [M + Na] ${ }^{+}$calcd for $\mathrm{C}_{24} \mathrm{H}_{38} \mathrm{O}_{6} \mathrm{Na}$ 445.2561; found 445.2550.

[1S, 4R,6R,7R,7(2S,3S,4R,5R),8S,12R]-7-[2-(2,5-Diethoxy-3,4-dihydroxytetrahydrofuran-3-yl)ethyl]-7-hydroxy -1,6,8-trimethyl-2-oxo-3-oxatricyclo[6.3.1.0 $\left.0^{4,12}\right]$ dodecane (38) and $[1 S, 4 R, 6 R, 7 R, 7(2 R, 3 R, 4 S, 5 S), 8 S$, 12R]-7-[2-(2,5-diethoxy-3,4-dihydroxytetrahydrofuran-3-yl)ethyl]-7-hydroxy-1,6,8-trimethyl-2-oxo-3-oxatri cyclo[6.3.1.0 $0^{4,12}$ ]dodecane (39). A $0.157 \mathrm{M}$ solution of $\mathrm{OsO}_{4}$ in $\mathrm{t}-\mathrm{BuOH}(0.06 \mathrm{~mL}, 9.4 \mu \mathrm{mol})$ was added to a solution of bisacetals $36(21.7 \mathrm{mg}, 51 \mu \mathrm{mol})$ and $\mathrm{NMO}\left(4.8 \mathrm{M}\right.$ in $\left.\mathrm{H}_{2} \mathrm{O}, 0.04 \mathrm{~mL}, 0.19 \mathrm{mmol}\right)$ in $\mathrm{THF} / \mathrm{H}_{2} \mathrm{O}$ (10:1, $0.55 \mathrm{~mL})$. After $1 \mathrm{~h}$ of stirring, the reaction was quenched with $1 \mathrm{M}$ aqueous $\mathrm{Na}_{2} \mathrm{~S}_{2} \mathrm{O}_{3}(5 \mathrm{~mL})$, and the resulting mixture was extracted with AcOEt $(2 \times 15 \mathrm{~mL})$. The combined organic extracts were washed with brine $(15 \mathrm{~mL})$, and dried over anhydrous $\mathrm{Na}_{2} \mathrm{SO}_{4}$. Filtration and evaporation in vacuo furnished the crude product $(29.1 \mathrm{mg})$, which was purified by flash column chromatography (silica gel 
$2.3 \mathrm{~g}, 1: 1 \mathrm{n}$-hexane/AcOEt) to give a 1:1 mixture of triols 38 and $39(14.3 \mathrm{mg}, 61 \%)$ as a colorless oil, along with recovered bisacetals $36(8.2 \mathrm{mg}, 38 \%)$ as a colorless oil. $R_{f} 0.49\left(1: 4 n\right.$-hexane/AcOEt); $[\alpha]_{D}^{21}$ +29.1 (c 0.56, $\mathrm{CHCl}_{3}$ ); IR (neat) 3462, 2930, 1751, 1458, 1389, 1375, 1101, $978 \mathrm{~cm}^{-1} ;{ }^{1} \mathrm{H}-\mathrm{NMR}(500 \mathrm{MHz}$, $\left.\mathrm{CDCl}_{3}\right) \delta 0.91\left(\mathrm{~d}, J=6.5 \mathrm{~Hz}, 1.5 \mathrm{H}, \mathrm{C} 8-\mathrm{CH}_{3}\right), 0.92\left(\mathrm{~d}, J=6.4 \mathrm{~Hz}, 1.5 \mathrm{H}, \mathrm{C} 8-\mathrm{CH}_{3}\right), 1.04\left(\mathrm{~s}, 3 \mathrm{H}, \mathrm{C} 10-\mathrm{CH}_{3}\right)$, $1.207\left(\mathrm{t}, J=7.1 \mathrm{~Hz}, 1.5 \mathrm{H}, \mathrm{OCH}_{2} \mathrm{CH}_{3}\right), 1.214\left(\mathrm{t}, J=7.1 \mathrm{~Hz}, 1.5 \mathrm{H}, \mathrm{OCH}_{2} \mathrm{CH}_{3}\right), 1.23(\mathrm{t}, J=7.1 \mathrm{~Hz}, 3 \mathrm{H}$, $\left.\mathrm{OCH}_{2} \mathrm{CH}_{3}\right), 1.29\left(\mathrm{~s}, 3 \mathrm{H}, \mathrm{C} 4-\mathrm{CH}_{3}\right), 1.42-1.52\left(\mathrm{~m}, 2 \mathrm{H}, \mathrm{CH}_{2}\right), 1.60-1.97\left(\mathrm{~m}, 8 \mathrm{H}, 4 \times \mathrm{CH}_{2}\right), 2.03-2.15(\mathrm{~m}$, 3.5H, $\left.\mathrm{CH}_{2}, \mathrm{C} 8-\mathrm{H}, \mathrm{OH}\right), 2.24(\mathrm{~d}, J=4.7 \mathrm{~Hz}, 1 \mathrm{H}, \mathrm{C} 5-\mathrm{H}), 2.33(\mathrm{br} \mathrm{s}, 0.5 \mathrm{H}, \mathrm{OH}), 2.99(\mathrm{~d}, J=6.9 \mathrm{~Hz}, 0.5 \mathrm{H}$, $\mathrm{OH}), 3.05(\mathrm{~m}, 1 \mathrm{H}, \mathrm{OH}), 3.44-3.57\left(\mathrm{~m}, 2.5 \mathrm{H}, \mathrm{OCH}_{2} \mathrm{CH}_{3}, \mathrm{OH}\right), 3.76-3.84\left(\mathrm{~m}, 2 \mathrm{H}, \mathrm{OCH}_{2} \mathrm{CH}_{3}\right), 3.96(\mathrm{~m}, 1 \mathrm{H}$, C14-H), 4.73 (br s, 1H, C6-H), 4.85 (s, 0.5H, C16-H), $4.86(\mathrm{~s}, 0.5 \mathrm{H}, \mathrm{C} 16-H), 4.97(\mathrm{~d}, J=4.3 \mathrm{~Hz}, 0.5 \mathrm{H}$, $\mathrm{C} 15-H), 4.98(\mathrm{~d}, J=4.3 \mathrm{~Hz}, 0.5 \mathrm{H}, \mathrm{C} 15-\mathrm{H}) ;{ }^{13} \mathrm{C}-\mathrm{NMR}\left(125.7 \mathrm{MHz}, \mathrm{CDCl}_{3}\right) \delta 14.88\left(\mathrm{CH}_{3}\right), 14.93\left(\mathrm{CH}_{3}\right)$, $15.2\left(\mathrm{CH}_{3}\right), 16.5\left(\mathrm{CH}_{3}\right), 16.8\left(\mathrm{CH}_{3}\right), 18.1\left(\mathrm{CH}_{2}\right), 22.2\left(\mathrm{CH}_{3}\right), 22.3\left(\mathrm{CH}_{3}\right), 22.85\left(\mathrm{CH}_{3}\right), 22.93\left(\mathrm{CH}_{3}\right), 27.4$ $\left(\mathrm{CH}_{2}\right), 28.1\left(\mathrm{CH}_{2}\right), 28.27\left(\mathrm{CH}_{2}\right), 28.28\left(\mathrm{CH}_{2}\right), 28.31\left(\mathrm{CH}_{2}\right), 28.4\left(\mathrm{CH}_{2}\right), 28.5\left(\mathrm{CH}_{2}\right), 28.6\left(\mathrm{CH}_{2}\right), 31.5\left(\mathrm{CH}_{2}\right)$, $31.6\left(\mathrm{CH}_{2}\right), 32.47(\mathrm{CH}), 32.49(\mathrm{CH}), 39.97(\mathrm{C}), 40.03(\mathrm{C}), 43.88(\mathrm{C}), 43.91(\mathrm{C}), 44.90(\mathrm{CH}), 44.93(\mathrm{CH})$, $63.07\left(\mathrm{CH}_{2}\right), 63.10\left(\mathrm{CH}_{2}\right), 64.55\left(\mathrm{CH}_{2}\right), 64.57\left(\mathrm{CH}_{2}\right), 75.30(\mathrm{C}), 75.33(\mathrm{C}), 76.3(\mathrm{CH}), 76.4(\mathrm{CH}), 80.3(\mathrm{CH})$, $80.4(\mathrm{CH}), 81.2(\mathrm{C}), 81.3(\mathrm{C}), 106.5(\mathrm{CH}), 106.7(\mathrm{CH}), 109.17(\mathrm{CH}), 109.19(\mathrm{CH}), 184.10(\mathrm{C}), 184.12(\mathrm{C})$; HRMS (ESI) $\mathrm{m} / \mathrm{z}[\mathrm{M}+\mathrm{Na}]^{+}$calcd for $\mathrm{C}_{24} \mathrm{H}_{40} \mathrm{O}_{8} \mathrm{Na}$ 479.2615; found 479.2631.

Marrulibacetal (13). TsOH (2.9 mg, $17 \mu \mathrm{mol})$ was added to a mixture of triols 38 and 39 (12.5 mg, 27 $\mu \mathrm{mol})$ in benzene $(1 \mathrm{~mL})$, and the mixture was stirred for $1.5 \mathrm{~h}$. The reaction was quenched with saturated aqueous $\mathrm{NaHCO}_{3}(5 \mathrm{~mL})$, and the resulting mixture was extracted with AcOEt $(2 \times 15 \mathrm{~mL})$. The combined organic extracts were washed with brine $(15 \mathrm{~mL})$, and dried over anhydrous $\mathrm{Na}_{2} \mathrm{SO}_{4}$. Filtration and evaporation in vacuo furnished the crude product $(19.2 \mathrm{mg})$, which was purified by flash column chromatography (silica gel $2 \mathrm{~g}, 2: 1 n$-hexane/AcOEt) to give a mixture of marrulibacetal (13) and its diastereomers. The mixture was flash chromatographed (silica gel $4.5 \mathrm{~g}, 30: 1 \mathrm{CHCl}_{3} /$ acetone) to provide a mixture of marrulibacetal (13) and C13,14,15,16-epimer 41, along with a 1:1 mixture of C15-epimer and C13,14,16-epimer (2.0 mg, 18\%). Separation of marrulibacetal (13) and 41 by flash column chromatography (silica gel $4.5 \mathrm{~g}$, 50:1 $\mathrm{CHCl}_{3}$ /acetone) yielded marrulibacetal $(\mathbf{1 3}, 4.1 \mathrm{mg}, 36 \%)$ and $\mathrm{C} 13,14,15,16$-epimer 41 (4.3 mg, 38\%) as white solids. $R_{f} 0.44\left(4: 1 \mathrm{CH}_{2} \mathrm{Cl}_{2} /\right.$ acetone); $\mathrm{mp} 177-179$ ${ }^{\circ} \mathrm{C}$ (colorless needles from $n$-hexane/benzene); $[\alpha]_{D}^{27}-21.7\left(c 1.16, \mathrm{CHCl}_{3}\right)$ [lit. [12], [ $\left.\alpha\right]_{D}^{25}-13.1$ (c 0.29, $\mathrm{CHCl}_{3}$ )]; IR (neat) 3435, 2961, 2928, 1773, 1740, 1458, 1389, 1244, 1200, 1111, 1053, $935 \mathrm{~cm}^{-1}$; ${ }^{1} \mathrm{H}-\mathrm{NMR}$ $\left(500 \mathrm{MHz}, \mathrm{CDCl}_{3}\right) \delta 1.03\left(\mathrm{~s}, 3 \mathrm{H}, \mathrm{C} 10-\mathrm{CH}_{3}\right), 1.11\left(\mathrm{~d}, \mathrm{~J}=6.8 \mathrm{~Hz}, 3 \mathrm{H}, \mathrm{C} 8-\mathrm{CH}_{3}\right), 1.19\left(\mathrm{~m}, 1 \mathrm{H}\right.$, one of $\left.\mathrm{C} 1-\mathrm{H}_{2}\right)$, $1.22\left(\mathrm{t}, J=7.1 \mathrm{~Hz}, 3 \mathrm{H}, \mathrm{OCH}_{2} \mathrm{CH}_{3}\right), 1.28\left(\mathrm{~s}, 3 \mathrm{H}, \mathrm{C} 4-\mathrm{CH}_{3}\right), 1.42\left(\mathrm{~m}, 1 \mathrm{H}\right.$, one of $\left.\mathrm{C} 3-\mathrm{H}_{2}\right), 1.49(\mathrm{~m}, 1 \mathrm{H}$, one of $\left.\mathrm{C} 2-\mathrm{H}_{2}\right), 1.71-1.78\left(\mathrm{~m}, 2 \mathrm{H}\right.$, one of $\mathrm{C} 2-\mathrm{H}_{2}$, one of $\left.\mathrm{C} 11-\mathrm{H}_{2}\right), 1.80-1.90\left(\mathrm{~m}, 3 \mathrm{H}\right.$, one of $\mathrm{C} 7-\mathrm{H}_{2}$, one of $\mathrm{C} 11-\mathrm{H}_{2}$, one of $\left.\mathrm{C} 12-\mathrm{H}_{2}\right), 1.98\left(\mathrm{~m}, 1 \mathrm{H}\right.$, one of $\left.\mathrm{C} 1-\mathrm{H}_{2}\right), 2.05-2.20\left(\mathrm{~m}, 4 \mathrm{H}\right.$, one of $\mathrm{C} 3-\mathrm{H}_{2}$, one of $\mathrm{C} 7-\mathrm{H}_{2}, \mathrm{C} 8-\mathrm{H}$, one of C12- $\left.\mathrm{H}_{2}\right), 2.36(\mathrm{~d}, J=4.7 \mathrm{~Hz}, 1 \mathrm{H}, \mathrm{C} 5-\mathrm{H}), 2.60(\mathrm{~s}, 1 \mathrm{H}, \mathrm{C} 13-\mathrm{OH}), 2.61(\mathrm{~d}, J=6.2 \mathrm{~Hz}, 1 \mathrm{H}, \mathrm{C} 14-\mathrm{OH}), 3.51(\mathrm{dq}$, $J=9.5,7.1 \mathrm{~Hz}, 1 \mathrm{H}$, one of $\left.\mathrm{OCH}_{2} \mathrm{CH}_{3}\right), 3.82\left(\mathrm{dq}, J=9.5,7.1 \mathrm{~Hz}, 1 \mathrm{H}\right.$, one of $\left.\mathrm{OCH}_{2} \mathrm{CH}_{3}\right), 3.95(\mathrm{dd}, J=2.0$, $6.2 \mathrm{~Hz}, 1 \mathrm{H}, \mathrm{C} 14-H), 4.77(\mathrm{br} \mathrm{dd}, J=4.7,5.9 \mathrm{~Hz}, 1 \mathrm{H}, \mathrm{C} 6-H), 5.04(\mathrm{~d}, J=2.0 \mathrm{~Hz}, 1 \mathrm{H}, \mathrm{C} 15-H), 5.46(\mathrm{~s}, 1 \mathrm{H}$, C16-H); ${ }^{13} \mathrm{C}-\mathrm{NMR}\left(125.7 \mathrm{MHz}, \mathrm{CDCl}_{3}\right) \delta 15.0\left(\mathrm{CH}_{3}\right), 17.9\left(\mathrm{CH}_{2}\right), 19.5\left(\mathrm{CH}_{3}\right), 21.1\left(\mathrm{CH}_{2}\right), 22.1\left(\mathrm{CH}_{3}\right)$, $23.1\left(\mathrm{CH}_{3}\right), 27.8\left(\mathrm{CH}_{2}\right), 28.2\left(\mathrm{CH}_{2}\right), 29.6\left(\mathrm{CH}_{2}\right), 32.3\left(\mathrm{CH}_{2}\right), 33.6(\mathrm{CH}), 40.9(\mathrm{C}), 43.9(\mathrm{C}), 44.6(\mathrm{CH}), 63.9$ $\left(\mathrm{CH}_{2}\right), 75.6(\mathrm{C}), 76.5(\mathrm{CH}), 78.5(\mathrm{CH}), 80.4(\mathrm{C}), 105.3(\mathrm{CH}), 108.7(\mathrm{CH}), 184.0(\mathrm{C})$; HRMS (EI) $\mathrm{m} / \mathrm{z}\left[\mathrm{M}^{+}\right.$] calcd for $\mathrm{C}_{22} \mathrm{H}_{34} \mathrm{O}_{7} 410.2305$; found 410.2300 .

Data for $\left(2 S, 2^{\prime} \mathrm{a} S, 3 S, 3 \mathrm{a} R, 5^{\prime} \mathrm{a} S, 6 R, 7^{\prime} R, 7_{\mathrm{a} R}, 8^{\prime} \mathrm{a} R, 8^{\prime} \mathrm{b} R\right)$-2-ethoxy-3,3a-dihydroxy-2'a $5^{\prime}$ a, $7^{\prime}$-trimethylt etradecahydrospiro[6H-furo[2,3- $b$ ]pyran-6,6'-[6H]naphtho[1,8-bc]furan]-2' $\left(2^{\prime} a H\right)$-one $(41) . R_{f} 0.56$ (4:1 $\mathrm{CH}_{2} \mathrm{Cl}_{2}$ /acetone); $[\alpha]_{D}^{28}+57.2$ (c 1.26, $\mathrm{CHCl}_{3}$ ); IR (KBr) 3458, 2930, 1771, 1749, 1466, 1389, 1260, 1198, 1153, 1094, 1063, 1040, 989, $941 \mathrm{~cm}^{-1} ;{ }^{1} \mathrm{H}-\mathrm{NMR}\left(500 \mathrm{MHz}, \mathrm{CDCl}_{3}\right) \delta 0.96\left(\mathrm{~d}, J=6.1 \mathrm{~Hz}, 3 \mathrm{H}, \mathrm{C} 8-\mathrm{CH}_{3}\right)$, $1.01\left(\mathrm{~s}, 3 \mathrm{H}, \mathrm{C} 10-\mathrm{CH}_{3}\right), 1.21\left(\mathrm{t}, J=7.1 \mathrm{~Hz}, 3 \mathrm{H}, \mathrm{OCH}_{2} \mathrm{CH}_{3}\right), 1.30\left(\mathrm{~s}, 3 \mathrm{H}, \mathrm{C} 4-\mathrm{CH}_{3}\right), 1.39-1.54(\mathrm{~m}, 4 \mathrm{H}$, one of $\mathrm{C} 1-\mathrm{H}_{2}$, one of $\mathrm{C} 2-\mathrm{H}_{2}$, one of $\mathrm{C} 3-\mathrm{H}_{2}$, one of $\left.\mathrm{C} 11-\mathrm{H}_{2}\right), 1.70-1.83\left(\mathrm{~m}, 4 \mathrm{H}\right.$, one of $\mathrm{C} 1-\mathrm{H}_{2}$, one of $\mathrm{C} 2-\mathrm{H}_{2}$, one of $\mathrm{C} 7-\mathrm{H}_{2}$, one of $\left.\mathrm{C} 12-\mathrm{H}_{2}\right), 1.95-2.11\left(\mathrm{~m}, 4 \mathrm{H}\right.$, one of $\mathrm{C} 3-\mathrm{H}_{2}$, one of $\mathrm{C} 7-\mathrm{H}_{2}, \mathrm{C} 8-\mathrm{H}$, one of $\left.\mathrm{C} 11-\mathrm{H}_{2}\right), 2.20$ $\left(\mathrm{m}, 1 \mathrm{H}\right.$, one of $\left.\mathrm{C} 12-\mathrm{H}_{2}\right), 2.34(\mathrm{~d}, J=4.5 \mathrm{~Hz}, 1 \mathrm{H}, \mathrm{C} 5-\mathrm{H}), 2.75(\mathrm{~d}, J=4.8 \mathrm{~Hz}, 1 \mathrm{H}, \mathrm{C} 14-\mathrm{OH}), 2.79(\mathrm{~s}, 1 \mathrm{H}$, $\mathrm{C} 13-\mathrm{OH}), 3.46\left(\mathrm{dq}, J=9.5,7.1 \mathrm{~Hz}, 1 \mathrm{H}\right.$, one of $\left.\mathrm{OCH}_{2} \mathrm{CH}_{3}\right), 3.74(\mathrm{~d}, J=4.8 \mathrm{~Hz}, 1 \mathrm{H}, \mathrm{C} 14-\mathrm{H}), 3.86(\mathrm{dq}, J=$ 9.5, 7.1 Hz, $1 \mathrm{H}$, one of $\left.\mathrm{OCH}_{2} \mathrm{CH}_{3}\right), 4.78(\mathrm{br} \mathrm{dd}, J=4.5,7.7 \mathrm{~Hz}, 1 \mathrm{H}, \mathrm{C} 6-\mathrm{H}), 5.01(\mathrm{~s}, 1 \mathrm{H}, \mathrm{C} 15-\mathrm{H}), 5.50$ (s, 
1H, C16-H); ${ }^{13} \mathrm{C}-\mathrm{NMR}\left(125.7 \mathrm{MHz}, \mathrm{CDCl}_{3}\right) \delta 14.8\left(\mathrm{CH}_{3}\right), 16.1\left(\mathrm{CH}_{3}\right), 18.1\left(\mathrm{CH}_{2}\right), 21.5\left(\mathrm{CH}_{2}\right), 23.2\left(\mathrm{CH}_{3}\right)$, $23.9\left(\mathrm{CH}_{3}\right), 28.3\left(\mathrm{CH}_{2}\right), 30.4\left(\mathrm{CH}_{2}\right), 30.9\left(\mathrm{CH}_{2}\right), 31.3\left(\mathrm{CH}_{2}\right), 35.0(\mathrm{CH}), 40.8(\mathrm{C}), 43.9(\mathrm{C}), 45.5(\mathrm{CH}), 63.7$ $\left(\mathrm{CH}_{2}\right), 75.9(\mathrm{C}), 76.7(\mathrm{CH}), 80.0(\mathrm{CH}), 80.5(\mathrm{C}), 106.1(\mathrm{CH}), 108.2(\mathrm{CH}), 183.9(\mathrm{C}) ; \mathrm{HRMS}(\mathrm{EI}) \mathrm{m} / \mathrm{z}\left[\mathrm{M}^{+}\right.$] calcd for $\mathrm{C}_{22} \mathrm{H}_{34} \mathrm{O}_{7} 410.2305$; found 410.2297 .

$[1 S, 4 R, 6 R, 7 R, 7(2 S, 3 S, 4 R, 5 R), 8 S, 12 R]-7-[2-(3,4-D i h y d r o x y-2,5-d i m e t h o x y$ tetrahydrofuran-3-yl)ethyl]-7-hydro $x y$-1,6,8-trimethyl-2-oxo-3-oxatricyclo[6.3.1. $\left.0^{4,12}\right]$ dodecane (40) and desertine (18). Pyridinium tribromide $(32.9 \mathrm{mg}, 0.102 \mathrm{mmol})$ was added to an ice-cooled $\left(0{ }^{\circ} \mathrm{C}\right)$ solution of marrubiin $(\mathbf{1}, 30.5 \mathrm{mg}, 91.7 \mu \mathrm{mol})$ in $\mathrm{CH}_{2} \mathrm{Cl}_{2} / \mathrm{MeOH}(1: 1,1.8 \mathrm{~mL})$. After $20 \mathrm{~min}$ of stirring, the reaction was quenched with saturated aqueous $\mathrm{NaHCO}_{3}(5 \mathrm{~mL})$, and the resulting mixture was extracted with AcOEt $(2 \times 15 \mathrm{~mL})$. The combined organic extracts were washed with brine $(2 \times 10 \mathrm{~mL})$, and dried over anhydrous $\mathrm{Na}_{2} \mathrm{SO}_{4}$. Filtration and evaporation in vacuo furnished the yellow oil (53.1 mg), which was purified by column chromatography (silica gel $3 \mathrm{~g}, 4: 1 \rightarrow 2: 1 \rightarrow 1: 1 n$-hexane/AcOEt) to give bisacetals 37 (33.1 mg, 91\%, dr $=2: 2: 1: 1)$ as a yellow oil.

A $0.157 \mathrm{M}$ solution of $\mathrm{OsO}_{4}$ in $t-\mathrm{BuOH}(0.08 \mathrm{~mL}, 12.5 \mu \mathrm{mol})$ was added to a solution of bisacetals $37(28.3 \mathrm{mg}, 71.7 \mu \mathrm{mol})$ and $\mathrm{NMO}\left(4.8 \mathrm{M}\right.$ in $\left.\mathrm{H}_{2} \mathrm{O}, 0.06 \mathrm{~mL}, 0.29 \mathrm{mmol}\right)$ in $\mathrm{THF} / \mathrm{H}_{2} \mathrm{O}(1: 1,0.8 \mathrm{~mL})$. After $1 \mathrm{~h}$ of stirring, the reaction was quenched with $1 \mathrm{M}$ aqueous $\mathrm{Na}_{2} \mathrm{~S}_{2} \mathrm{O}_{3}(5 \mathrm{~mL})$, and the resulting mixture was extracted with AcOEt $(2 \times 15 \mathrm{~mL})$. The combined organic extracts were washed with brine $(2 \times 10$ $\mathrm{mL}$ ), and dried over anhydrous $\mathrm{Na}_{2} \mathrm{SO}_{4}$. Filtration and evaporation in vacuo furnished the brown oil ( $40.7 \mathrm{mg}$ ), which was purified by column chromatography (silica gel 3.1 g, 2:1 $\rightarrow$ 1:1 $n$-hexane/AcOEt) to give a 1:1 mixture of desertine (18) and its diastereomer $40(20.2 \mathrm{mg}, 66 \%)$ as a brown oil, along with recovered bisacetals $37(8.8 \mathrm{mg}, 29 \%)$ as a colorless oil. $R_{f} 0.30(1: 1 n$-hexane/AcOEt $) ;[\alpha]_{D}^{20}+12.9(c 1.02$, $\mathrm{CHCl}_{3}$ ); IR (neat) 3464, 2951, 1748, 1454, 1391, 1258, 1198, 1148, 1101, 1043, $989 \mathrm{~cm}^{-1} ;{ }^{1} \mathrm{H}-\mathrm{NMR}$ (500 $\left.\mathrm{MHz}, \mathrm{CDCl}_{3}\right) \delta 0.91\left(\mathrm{~d}, \mathrm{~J}=6.5 \mathrm{~Hz}, 3 \mathrm{H}, \mathrm{C} 8-\mathrm{CH}_{3}\right), 1.04\left(\mathrm{~s}, 1.5 \mathrm{H}, \mathrm{C} 10-\mathrm{CH}_{3}\right), 1.05\left(\mathrm{~s}, 1.5 \mathrm{H}, \mathrm{C}_{10}-\mathrm{CH}_{3}\right), 1.28$ $\left(\mathrm{m}, 1 \mathrm{H}\right.$, one of $\left.\mathrm{C} 1-\mathrm{H}_{2}\right), 1.29\left(\mathrm{~s}, 3 \mathrm{H}, \mathrm{C} 4-\mathrm{CH}_{3}\right), 1.45\left(\mathrm{~m}, 1 \mathrm{H}\right.$, one of $\left.\mathrm{C} 3-\mathrm{H}_{2}\right), 1.51\left(\mathrm{~m}, 1 \mathrm{H}\right.$, one of $\left.\mathrm{C} 2-\mathrm{H}_{2}\right)$, $1.65\left(\mathrm{~m}, 1 \mathrm{H}\right.$, one of $\left.\mathrm{C} 7-\mathrm{H}_{2}\right), 1.60-1.98\left(\mathrm{~m}, 6 \mathrm{H}\right.$, one of $\mathrm{C} 1-\mathrm{H}_{2}$, one of $\left.\mathrm{C} 2-\mathrm{H}_{2}, \mathrm{C} 11-\mathrm{H}_{2}, \mathrm{C} 12-\mathrm{H}_{2}\right), 2.07(\mathrm{~m}$, $1 \mathrm{H}, \mathrm{C} 8-\mathrm{H}), 2.11\left(\mathrm{~m}, 1 \mathrm{H}\right.$, one of $\left.\mathrm{C} 3-\mathrm{H}_{2}\right), 2.14\left(\mathrm{~m}, 1 \mathrm{H}\right.$, one of $\left.\mathrm{C} 7-\mathrm{H}_{2}\right), 2.22(\mathrm{~d}, J=4.2 \mathrm{~Hz}, 0.5 \mathrm{H}, \mathrm{C} 5-\mathrm{H}), 2.23$ $(\mathrm{d}, J=4.2 \mathrm{~Hz}, 0.5 \mathrm{H}, \mathrm{C} 5-\mathrm{H}), 3.40\left(\mathrm{~s}, 3 \mathrm{H}, \mathrm{C} 16-\mathrm{OCH}_{3}\right), 3.47\left(\mathrm{~s}, 3 \mathrm{H}, \mathrm{C} 15-\mathrm{OCH}_{3}\right), 3.92(\mathrm{~d}, J=3.5 \mathrm{~Hz}, 0.5 \mathrm{H}$, C14-H), $3.95(\mathrm{~d}, J=3.5 \mathrm{~Hz}, 0.5 \mathrm{H}, \mathrm{C} 14-H), 4.73(\mathrm{~m}, 1 \mathrm{H}, \mathrm{C} 6-H), 4.76(\mathrm{~s}, 0.5 \mathrm{H}, \mathrm{C} 16-H), 4.77(\mathrm{~s}, 0.5 \mathrm{H}, \mathrm{C} 16-H)$, $4.89(\mathrm{~d}, J=3.5 \mathrm{~Hz}, 0.5 \mathrm{H}, \mathrm{C} 15-\mathrm{H}), 4.90(\mathrm{~d}, J=3.5 \mathrm{~Hz}, 0.5 \mathrm{H}, \mathrm{C} 15-H) ;{ }^{13} \mathrm{C}-\mathrm{NMR}\left(125.7 \mathrm{MHz}, \mathrm{CDCl}_{3}\right) \delta 16.4$ $\left(\mathrm{CH}_{3}\right), 16.8\left(\mathrm{CH}_{3}\right), 18.1\left(\mathrm{CH}_{2}\right), 22.2\left(\mathrm{CH}_{3}\right), 22.3\left(\mathrm{CH}_{3}\right), 22.86\left(\mathrm{CH}_{3}\right), 22.94\left(\mathrm{CH}_{3}\right), 27.4\left(\mathrm{CH}_{2}\right), 28.0\left(\mathrm{CH}_{2}\right)$, $28.2\left(\mathrm{CH}_{2}\right), 28.26\left(\mathrm{CH}_{2}\right), 28.28\left(\mathrm{CH}_{2}\right), 28.41\left(\mathrm{CH}_{2}\right), 28.43\left(\mathrm{CH}_{2}\right), 28.5\left(\mathrm{CH}_{2}\right), 31.5\left(\mathrm{CH}_{2}\right), 31.6\left(\mathrm{CH}_{2}\right), 32.5$ $(\mathrm{CH}), 32.6(\mathrm{CH}), 40.0(\mathrm{C}), 40.1(\mathrm{C}), 43.87(\mathrm{C}), 43.91(\mathrm{C}), 44.9(\mathrm{CH}), 45.0(\mathrm{CH}), 55.08\left(\mathrm{CH}_{3}\right), 55.11\left(\mathrm{CH}_{3}\right)$, $56.40\left(\mathrm{CH}_{3}\right), 56.42\left(\mathrm{CH}_{3}\right), 75.3(\mathrm{C}), 75.4(\mathrm{C}), 76.2(\mathrm{CH}), 76.3(\mathrm{CH}), 80.2(\mathrm{CH}), 80.3(\mathrm{CH}), 81.2(\mathrm{C}), 81.4(\mathrm{C})$, $108.3(\mathrm{CH}), 108.6(\mathrm{CH}), 110.7(\mathrm{CH}), 110.8(\mathrm{CH}), 183.95(\mathrm{C}), 183.99(\mathrm{C})$; HRMS (ESI) $m / z\left[\mathrm{M}+\mathrm{Na}^{+} \mathrm{calcd}^{2}\right.$ for $\mathrm{C}_{22} \mathrm{H}_{36} \mathrm{O}_{8} \mathrm{Na} 451.2302$; found 451.2308 .

Marrulibacetal A (17). $\mathrm{TsOH}(8.6 \mathrm{mg}, 50 \mu \mathrm{mol})$ was added to a mixture of desertine (18) and its diastereomer $40(29.6 \mathrm{mg}, 69.0 \mu \mathrm{mol})$ in benzene $(1.4 \mathrm{~mL})$. After $5 \mathrm{~h}$ of stirring, an additional portion of $\mathrm{TsOH}(1.6 \mathrm{mg}, 9.3 \mu \mathrm{mol})$ was added, and the reaction mixture was stirred for $3.5 \mathrm{~h}$. The reaction was quenched with saturated aqueous $\mathrm{NaHCO}_{3}(2 \mathrm{~mL})$, and the resulting mixture was extracted with AcOEt $(3 \times 5 \mathrm{~mL})$. The combined organic extracts were washed with brine $(5 \mathrm{~mL})$, and dried over anhydrous $\mathrm{Na}_{2} \mathrm{SO}_{4}$. Filtration and evaporation in vacuo furnished the brown oil (68.1 mg), which was purified by flash column chromatography (silica gel $5 \mathrm{~g}, 1: 1 n$-hexane/AcOEt) to give marrulibacetal A (17, $4.3 \mathrm{mg}, 16 \%)$ and C13,14,15,16-isomer $42(6.2 \mathrm{mg}, 23 \%)$ as colorless amorphous solids. $R_{f} 0.73$ (AcOEt); $[\alpha]_{D}^{21}-14.0$ (c 1.69, $\mathrm{CHCl}_{3}$ ) (lit. [14], [ $\left.\left.\alpha\right]_{D}^{25}-10.77\right)$; IR (neat) 2953, 2928, 1769, 1748, 1456, 1259, 1198, 1117, 1053, 1015, 989, $935 \mathrm{~cm}^{-1} ;{ }^{1} \mathrm{H}-\mathrm{NMR}\left(500 \mathrm{MHz}, \mathrm{CDCl}_{3}\right) \delta 1.03\left(\mathrm{~s}, 3 \mathrm{H}, \mathrm{C} 10-\mathrm{CH}_{3}\right), 1.11(\mathrm{~d}, \mathrm{~J}$ $\left.=7.0 \mathrm{~Hz}, 3 \mathrm{H}, \mathrm{C} 8-\mathrm{CH}_{3}\right), 1.19\left(\mathrm{~m}, 1 \mathrm{H}\right.$, one of $\left.\mathrm{C}^{-}-\mathrm{H}_{2}\right), 1.28\left(\mathrm{~s}, 3 \mathrm{H}, \mathrm{C} 4-\mathrm{CH}_{3}\right), 1.42\left(\mathrm{~m}, 1 \mathrm{H}\right.$, one of $\left.\mathrm{C} 3-\mathrm{H}_{2}\right)$, $1.49\left(\mathrm{~m}, 1 \mathrm{H}\right.$, one of $\left.\mathrm{C} 2-\mathrm{H}_{2}\right), 1.71-1.92\left(\mathrm{~m}, 5 \mathrm{H}\right.$, one of $\mathrm{C} 2-\mathrm{H}_{2}$, one of $\mathrm{C} 7-\mathrm{H}_{2}, \mathrm{C} 11-\mathrm{H}_{2}$, one of $\left.\mathrm{C} 12-\mathrm{H}_{2}\right)$, $1.96\left(\mathrm{t}, J=10.6 \mathrm{~Hz}, 1 \mathrm{H}\right.$, one of $\left.\mathrm{C} 1-\mathrm{H}_{2}\right), 2.05-2.15\left(\mathrm{~m}, 3 \mathrm{H}\right.$, one of $\mathrm{C} 3-\mathrm{H}_{2}, \mathrm{C} 8-\mathrm{H}$, one of $\left.\mathrm{C} 12-\mathrm{H}_{2}\right), 2.20$ $\left(\mathrm{dd}, J=5.5,16.0 \mathrm{~Hz}, 1 \mathrm{H}\right.$, one of $\left.\mathrm{C} 7-\mathrm{H}_{2}\right), 2.38(\mathrm{~d}, J=4.5 \mathrm{~Hz}, 1 \mathrm{H}, \mathrm{C} 5-H), 3.42\left(\mathrm{~s}, 3 \mathrm{H}, \mathrm{OCH}_{3}\right), 3.89(\mathrm{~d}, J=$ 
$1.2 \mathrm{~Hz}, 1 \mathrm{H}, \mathrm{C} 14-H), 4.79(\mathrm{dd}, J=4.5,6.3 \mathrm{~Hz}, 1 \mathrm{H}, \mathrm{C} 6-H), 4.93(\mathrm{~d}, J=1.2 \mathrm{~Hz}, 1 \mathrm{H}, \mathrm{C} 15-H), 5.48(\mathrm{~s}, 1 \mathrm{H}$, C16-H); ${ }^{13} \mathrm{C}-\mathrm{NMR}\left(125.7 \mathrm{MHz}, \mathrm{CDCl}_{3}\right) \delta 17.9\left(\mathrm{CH}_{2}\right), 19.4\left(\mathrm{CH}_{3}\right), 20.7\left(\mathrm{CH}_{2}\right), 22.0\left(\mathrm{CH}_{3}\right), 23.1\left(\mathrm{CH}_{3}\right)$, 27.8 $\left(\mathrm{CH}_{2}\right), 28.2\left(\mathrm{CH}_{2}\right), 30.0\left(\mathrm{CH}_{2}\right), 32.2\left(\mathrm{CH}_{2}\right), 33.5(\mathrm{CH}), 40.9(\mathrm{C}), 43.9(\mathrm{C}), 44.6(\mathrm{CH}), 55.5\left(\mathrm{CH}_{3}\right), 75.8$ (C), $76.5(\mathrm{CH}), 78.6(\mathrm{CH}), 80.3(\mathrm{C}), 105.7(\mathrm{CH}), 109.8(\mathrm{CH}), 184.1(\mathrm{C})$; HRMS (ESI) $\mathrm{m} / z$ [M + Na] ${ }^{+}$calcd for $\mathrm{C}_{21} \mathrm{H}_{32} \mathrm{O}_{7} \mathrm{Na} 419.2045$; found 419.2037 .

Data for ( $\left.2 S, 2^{\prime} \mathrm{a} S, 3 S, 3 \mathrm{a} R, 5^{\prime} \mathrm{a} S, 6 R, 7^{\prime} R, 7 \mathrm{a} R, 8^{\prime} \mathrm{a} R, 8^{\prime} \mathrm{b} R\right)$-3,3a-dihydroxy-2-methoxy-2' a, $5^{\prime}$ a, $7^{\prime}$-trimethyl tetradecahydrospiro[6H-furo[2,3-b]pyran-6,6'-[6H]naphtho[1,8-bc]furan]-2' $\left(2^{\prime} \mathrm{a} H\right)$-one $(42): R_{f} 0.78$ (AcOEt); $[\alpha]_{D}^{22}+49.1$ (c 0.75, $\mathrm{CHCl}_{3}$ ); IR (neat) 2955, 2928, 1749, 1541, 1506, 1456, $1265 \mathrm{~cm}^{-1} ;{ }^{1} \mathrm{H}-\mathrm{NMR}$ $\left(500 \mathrm{MHz}, \mathrm{CDCl}_{3}\right) \delta 0.94\left(\mathrm{~d}, J=6.1 \mathrm{~Hz}, 3 \mathrm{H}, \mathrm{C} 8-\mathrm{CH}_{3}\right), 1.01\left(\mathrm{~s}, 3 \mathrm{H}, \mathrm{C} 10-\mathrm{CH}_{3}\right), 1.31\left(\mathrm{~s}, 3 \mathrm{H}, \mathrm{C} 4-\mathrm{CH}_{3}\right)$, 1.42-1.56 (m, $4 \mathrm{H}$, one of $\mathrm{C} 1-\mathrm{H}_{2}$, one of $\mathrm{C} 2-\mathrm{H}_{2}$, one of $\mathrm{C} 3-\mathrm{H}_{2}$, one of $\left.\mathrm{C} 11-\mathrm{H}_{2}\right), 1.70-1.83(\mathrm{~m}, 4 \mathrm{H}$, one of $\mathrm{C} 1-\mathrm{H}_{2}$, one of $\mathrm{C} 2-\mathrm{H}_{2}$, one of $\mathrm{C} 7-\mathrm{H}_{2}$, one of $\left.\mathrm{C} 12-\mathrm{H}_{2}\right), 1.98\left(\mathrm{dt}, \mathrm{J}=3.8,14.2 \mathrm{~Hz}, 1 \mathrm{H}\right.$, one of $\left.\mathrm{C} 11-\mathrm{H}_{2}\right)$, 1.99-2.12 (m, 3H, one of C3- $\mathrm{H}_{2}$, one of $\left.\mathrm{C} 7-\mathrm{H}_{2}, \mathrm{C} 8-\mathrm{H}\right), 2.17\left(\mathrm{dt}, J=4.4,14.2 \mathrm{~Hz}, 1 \mathrm{H}\right.$, one of $\left.\mathrm{C} 12-\mathrm{H}_{2}\right), 2.35$ $(\mathrm{d}, J=4.5 \mathrm{~Hz}, 1 \mathrm{H}, \mathrm{C} 5-H), 2.87(\mathrm{br} \mathrm{s}, 1 \mathrm{H}, \mathrm{OH}), 3.41\left(\mathrm{~s}, 3 \mathrm{H}, \mathrm{OCH}_{3}\right), 3.73(\mathrm{~s}, 1 \mathrm{H}, \mathrm{C} 14-H), 4.79(\mathrm{dd}, J=4.5$, $6.2 \mathrm{~Hz}, 1 \mathrm{H}, \mathrm{C} 6-H), 4.91(\mathrm{~s}, 1 \mathrm{H}, \mathrm{C} 15-\mathrm{H}), 5.52(\mathrm{~s}, 1 \mathrm{H}, \mathrm{C} 16-H) ;{ }^{13} \mathrm{C}-\mathrm{NMR}\left(125.7 \mathrm{MHz}, \mathrm{CDCl}_{3}\right) \delta 16.0\left(\mathrm{CH}_{3}\right)$, 18.1 $\left(\mathrm{CH}_{2}\right), 21.4\left(\mathrm{CH}_{2}\right), 23.1\left(\mathrm{CH}_{3}\right), 24.0\left(\mathrm{CH}_{3}\right), 28.3\left(\mathrm{CH}_{2}\right), 30.6\left(\mathrm{CH}_{2}\right), 30.9\left(\mathrm{CH}_{2}\right), 31.3\left(\mathrm{CH}_{2}\right), 35.0(\mathrm{CH})$, $40.8(\mathrm{C}), 43.9(\mathrm{C}), 45.5(\mathrm{CH}), 55.2\left(\mathrm{CH}_{3}\right), 76.1(\mathrm{C}), 76.7(\mathrm{CH}), 79.8(\mathrm{CH}), 80.5(\mathrm{C}), 106.3(\mathrm{CH}), 109.4(\mathrm{CH})$, 184.1 (C); HRMS (ESI) $m / z$ [M + Na] ${ }^{+}$calcd for $\mathrm{C}_{21} \mathrm{H}_{32} \mathrm{O}_{7} \mathrm{Na}$ 419.2045; found 419.2059.

Marrubasch F (19). $m$-CPBA (ca. 70\%, $10.7 \mathrm{mg}, 43.4 \mu \mathrm{mol}$ ) was azeotropically dried with benzene, and dissolved in $\mathrm{CH}_{2} \mathrm{Cl}_{2}(0.2 \mathrm{~mL})$. The $m$-CPBA solution was added to an ice-cooled $\left(0{ }^{\circ} \mathrm{C}\right)$ solution of silylated marrubiin $35(8.1 \mathrm{mg}, 20.0 \mu \mathrm{mol})$ in $\mathrm{CH}_{2} \mathrm{Cl}_{2}(0.8 \mathrm{~mL})$. After $1.5 \mathrm{~h}$ of stirring, the reaction was quenched with a mixture of $1 \mathrm{M}$ aqueous $\mathrm{Na}_{2} \mathrm{~S}_{2} \mathrm{O}_{3}(2 \mathrm{~mL})$ and saturated aqueous $\mathrm{NaHCO}_{3}(2 \mathrm{~mL})$, and the resulting mixture was extracted with AcOEt $(10 \mathrm{~mL})$. The organic extract was washed with brine (4 $\mathrm{mL}$ ), and dried over anhydrous $\mathrm{Na}_{2} \mathrm{SO}_{4}$. Filtration and evaporation in vacuo furnished the colorless oil (28.4 mg), which was purified by preparative thin layer chromatography $(200 \mathrm{~mm} \times 100 \mathrm{~mm} \times 0.25$ $\mathrm{mm}$ preparative silica gel plate and elution with 1:1 $n$-hexane/AcOEt) to give marrubasch $\mathrm{F}(\mathbf{1 9}, 3.9$ $\mathrm{mg}, 56 \%)$ as a white solid. $R_{f} 0.32\left(1: 1 n\right.$-hexane/AcOEt); $\mathrm{mp} 195-196{ }^{\circ} \mathrm{C}$ (pale yellow plates from 1:2 $n$-hexane/AcOEt) (lit. [20], mp 191-193 $\left.{ }^{\circ} \mathrm{C}\right)$; $[\alpha]_{D}^{21}+41.1\left(c 0.534, \mathrm{CHCl}_{3}\right)$ [lit. [20], $[\alpha]_{D}+41.5(c 1.00$, $\mathrm{CHCl}_{3}$ )]; IR (neat) 3536, 2926, 2873, 1747, 1456, 1199, 1139, 1095, 1076, 1041, $983 \mathrm{~cm}^{-1} ;{ }^{1} \mathrm{H}-\mathrm{NMR}(500$ $\left.\mathrm{MHz}, \mathrm{CDCl}_{3}\right) \delta 0.96\left(\mathrm{~d}, J=6.4 \mathrm{~Hz}, 3 \mathrm{H}, \mathrm{C} 8-\mathrm{CH}_{3}\right), 1.04\left(\mathrm{~s}, 3 \mathrm{H}, \mathrm{C} 10-\mathrm{CH}_{3}\right), 1.25(\mathrm{dt}, J=12.8,8.5 \mathrm{~Hz}, 1 \mathrm{H}$, one of $\left.\mathrm{C} 1-\mathrm{H}_{2}\right), 1.30\left(\mathrm{~s}, 3 \mathrm{H}, \mathrm{C} 4-\mathrm{CH}_{3}\right), 1.45\left(\mathrm{dt}, J=14.4,4.8 \mathrm{~Hz}, 1 \mathrm{H}\right.$, one of $\left.\mathrm{C} 3-\mathrm{H}_{2}\right), 1.53\left(\mathrm{~m}, 1 \mathrm{H}\right.$, one of $\left.\mathrm{C} 2-\mathrm{H}_{2}\right)$, 1.69-1.84 (m, 5H, one of $\mathrm{C} 1-\mathrm{H}_{2}$, one of $\mathrm{C} 2-\mathrm{H}_{2}$, one of $\left.\mathrm{C} 7-\mathrm{H}_{2}, \mathrm{C} 11-\mathrm{H}_{2}\right), 2.05-2.17\left(\mathrm{~m}, 3 \mathrm{H}\right.$, one of $\mathrm{C} 3-\mathrm{H}_{2}$, one of C7- $\left.\mathrm{H}_{2}, \mathrm{C} 8-\mathrm{H}\right), 2.28(\mathrm{~d}, J=4.5 \mathrm{~Hz}, 1 \mathrm{H}, \mathrm{C} 5-\mathrm{H}), 2.41-2.52\left(\mathrm{~m}, 2 \mathrm{H}, \mathrm{C} 12-\mathrm{H}_{2}\right), 4.75(\mathrm{dd}, J=4.5,6.5 \mathrm{~Hz}$, $1 \mathrm{H}, \mathrm{C} 6-H), 4.80\left(\mathrm{br} \mathrm{d}, J=1.4 \mathrm{~Hz}, 2 \mathrm{H}, \mathrm{C} 15-\mathrm{H}_{2}\right), 7.14(\mathrm{t}, J=1.4 \mathrm{~Hz}, 1 \mathrm{H}, \mathrm{C} 14-H) ;{ }^{13} \mathrm{C}-\mathrm{NMR}(125.7 \mathrm{MHz}$, $\left.\mathrm{CDCl}_{3}\right) \delta 16.7\left(\mathrm{CH}_{3}\right), 18.1\left(\mathrm{CH}_{2}\right), 21.2\left(\mathrm{CH}_{2}\right), 22.3\left(\mathrm{CH}_{3}\right), 22.9\left(\mathrm{CH}_{3}\right), 28.3\left(\mathrm{CH}_{2}\right), 28.6\left(\mathrm{CH}_{2}\right), 31.6\left(\mathrm{CH}_{2}\right)$, $32.4\left(\mathrm{CH}_{2}\right), 32.6\left(\mathrm{CH}_{2}\right), 39.9(\mathrm{C}), 43.8(\mathrm{C}), 44.9(\mathrm{CH}), 70.4\left(\mathrm{CH}_{2}\right), 75.2(\mathrm{C}), 76.2(\mathrm{CH}), 134.7(\mathrm{C}), 144.3$ (CH), 174.9 (C), 183.9 (C); HRMS (ESI) $\mathrm{m} / z$ [M + Na] ${ }^{+}$calcd for $\mathrm{C}_{20} \mathrm{H}_{28} \mathrm{O}_{5} \mathrm{Na}$ 371.1830; found 371.1834.

(1S,4R,6R,7R,8S,12R)-7-Hydroxy-1,6,8-trimethyl-2-oxo-7-propargyl-3-oxatricyclo[6.3.1.0 $\left.0^{4,12}\right]$ dodecane (43). Lithium acetylide ethylene diamine complex $(36.5 \mathrm{mg}, 0.397 \mathrm{mmol})$ was added to a solution of epoxide $22(10.7 \mathrm{mg}, 42.7 \mu \mathrm{mol})$ in DMSO $(0.2 \mathrm{~mL})$. After $30 \mathrm{~min}$ of stirring, the reaction was quenched with $\mathrm{H}_{2} \mathrm{O}$ $(1 \mathrm{~mL})$, and the resulting mixture was extracted with AcOEt $(3 \times 5 \mathrm{~mL})$. The combined organic extracts were washed with brine $(2 \times 5 \mathrm{~mL})$, and dried over anhydrous $\mathrm{Na}_{2} \mathrm{SO}_{4}$. Filtration and evaporation in vacuo furnished the pale yellow solid $(13.1 \mathrm{mg})$, which was purified by column chromatography (silica gel $3 \mathrm{~g}$, 2:1 $n$-hexane/AcOEt) to give alcohol $43(11.2 \mathrm{mg}, 95 \%)$ as a pale yellow solid. $R_{f} 0.57$ (1:1 $n$-hexane/AcOEt); $\mathrm{mp} 195-196{ }^{\circ} \mathrm{C}$ (colorless plates from $n$-hexane/Et $\left.{ }_{2} \mathrm{O}\right) ;[\alpha]_{D}^{25}+29.9\left(c 0.249, \mathrm{CHCl}_{3}\right)$; IR (neat) 3306, 3019, 2955, 2934, 2872, 1757, 1458, 1427, 1393, 1136, 1096, 1045, $1003 \mathrm{~cm}^{-1}$; ${ }^{1} \mathrm{H}-\mathrm{NMR}$ (500 $\left.\mathrm{MHz}, \mathrm{CDCl}_{3}\right) \delta 0.99\left(\mathrm{~d}, J=6.3 \mathrm{~Hz}, 3 \mathrm{H}, \mathrm{C} 8-\mathrm{CH}_{3}\right), 1.05\left(\mathrm{~s}, 3 \mathrm{H}, \mathrm{C} 10-\mathrm{CH}_{3}\right), 1.25(\mathrm{dt}, J=13.0,9.0 \mathrm{~Hz}, 1 \mathrm{H}$, one of $\left.\mathrm{C} 1-\mathrm{H}_{2}\right), 1.30\left(\mathrm{~s}, 3 \mathrm{H}, \mathrm{C} 4-\mathrm{CH}_{3}\right), 1.46\left(\mathrm{dt}, J=14.7,4.2 \mathrm{~Hz}, 1 \mathrm{H}\right.$, one of $\left.\mathrm{C} 3-\mathrm{H}_{2}\right), 1.53(\mathrm{~m}, 1 \mathrm{H}$, one of C2- $\left.\mathrm{H}_{2}\right), 1.69\left(\mathrm{ddd}, J=6.3,13.3,17.7 \mathrm{~Hz}, 1 \mathrm{H}\right.$, one of $\left.\mathrm{C} 7-\mathrm{H}_{2}\right), 1.76\left(\mathrm{~m}, 1 \mathrm{H}\right.$, one of $\left.\mathrm{C} 2-\mathrm{H}_{2}\right), 1.85(\mathrm{dd}, J=9.6$, $11.6 \mathrm{~Hz}, 1 \mathrm{H}$, one of $\left.\mathrm{C} 1-\mathrm{H}_{2}\right), 2.09-2.18\left(\mathrm{~m}, 3 \mathrm{H}\right.$, one of $\mathrm{C} 3-\mathrm{H}_{2}$, one of $\left.\mathrm{C} 7-\mathrm{H}_{2}, \mathrm{C} 8-\mathrm{H}\right), 2.19(\mathrm{t}, J=2.7 \mathrm{~Hz}, 1 \mathrm{H}$, 
C13-H), $2.26(\mathrm{~d}, J=4.5 \mathrm{~Hz}, 1 \mathrm{H}, \mathrm{C} 5-H), 2.36\left(\mathrm{dd}, J=2.7,17.1 \mathrm{~Hz}, 1 \mathrm{H}\right.$, one of $\left.\mathrm{C} 11-H_{2}\right), 2.59(\mathrm{dd}, J=2.7$, $17.1 \mathrm{~Hz}, 1 \mathrm{H}$, one of C11- $\left.\mathrm{H}_{2}\right), 4.73(\mathrm{dd}, J=4.5,6.3 \mathrm{~Hz}, 1 \mathrm{H}, \mathrm{C} 6-\mathrm{H}) ;{ }^{13} \mathrm{C}-\mathrm{NMR}\left(125.7 \mathrm{MHz}, \mathrm{CDCl}_{3}\right) \delta 16.3$ $\left(\mathrm{CH}_{3}\right), 18.1\left(\mathrm{CH}_{2}\right), 22.3\left(\mathrm{CH}_{3}\right), 22.6\left(\mathrm{CH}_{3}\right), 25.3\left(\mathrm{CH}_{2}\right), 28.21\left(\mathrm{CH}_{2}\right), 28.22\left(\mathrm{CH}_{2}\right), 31.5\left(\mathrm{CH}_{2}\right), 32.3(\mathrm{CH})$, $39.2(\mathrm{C}), 43.9(\mathrm{C}), 44.8(\mathrm{CH}), 73.77(\mathrm{C}), 73.79(\mathrm{CH}), 76.1(\mathrm{CH}), 80.7(\mathrm{C}), 183.8(\mathrm{C})$; HRMS (ESI) $\mathrm{m} / \mathrm{z}$ [M + $\mathrm{Na}]^{+}$calcd for $\mathrm{C}_{17} \mathrm{H}_{24} \mathrm{O}_{3} \mathrm{Na} 299.1618$; found 299.1623 .

Cyllenine $C$ (12). To an ice-cooled $\left(0{ }^{\circ} \mathrm{C}\right)$ mixture of alcohol $43(6.8 \mathrm{mg}, 24.6 \mu \mathrm{mol})$ and $m$-CPBA (ca. $65 \%, 9.8 \mathrm{mg}, 36.9 \mu \mathrm{mol})$ in $\mathrm{CH}_{2} \mathrm{Cl}_{2}(0.3 \mathrm{~mL})$ was added a $0.123 \mathrm{M}$ solution of methanesulfonic acid in $\mathrm{CH}_{2} \mathrm{Cl}_{2}(0.20 \mathrm{~mL}, 24.6 \mu \mathrm{mol})$ followed by $\mathrm{Ph}_{3} \mathrm{PAuNTf}_{2}(1.0 \mathrm{mg}, 1.3 \mu \mathrm{mol})$. After $1 \mathrm{~h}$ of stirring at room temperature, the reaction was quenched with saturated aqueous $\mathrm{NaHCO}_{3}(1 \mathrm{~mL})$, and the resulting mixture was extracted with AcOEt $(2 \times 5 \mathrm{~mL})$. The combined organic extracts were washed with brine $(5 \mathrm{~mL})$, and dried over anhydrous $\mathrm{Na}_{2} \mathrm{SO}_{4}$. Filtration and evaporation in vacuo furnished the colorless oil (13.7 mg), which was purified by column chromatography (silica gel $2 \mathrm{~g}, 2: 1 \mathrm{n}$-hexane/AcOEt) to give cyllenine $\mathrm{C}(\mathbf{1 2}, 6.9 \mathrm{mg}, 96 \%)$ as a white solid. $R_{f} 0.32(1: 2 n$-hexane/AcOEt); mp 164-165 ${ }^{\circ} \mathrm{C}$ (colorless needles from $n$-hexane/ $\left.\mathrm{Et}_{2} \mathrm{O}\right) ;[\alpha]_{D}^{24}+22.1\left(c 0.69, \mathrm{CH}_{2} \mathrm{Cl}_{2}\right)\left[\right.$ lit. [11], $[\alpha]_{D}^{20}+11.82(c 0.33$, $\mathrm{CH}_{2} \mathrm{Cl}_{2}$ )]; IR (neat) 3019, 2934, 2870, 1763, 1474, 1458, 1420, 1391, 1273, 1120, 1067, 1042, $993 \mathrm{~cm}^{-1}$; ${ }^{1} \mathrm{H}-\mathrm{NMR}\left(500 \mathrm{MHz}, \mathrm{CDCl}_{3}\right) \delta 0.93\left(\mathrm{~d}, J=6.5 \mathrm{~Hz}, 3 \mathrm{H}, \mathrm{C} 8-\mathrm{CH}_{3}\right), 1.09\left(\mathrm{~s}, 3 \mathrm{H}, \mathrm{C} 10-\mathrm{CH}_{3}\right), 1.30(\mathrm{dt}, J=$ $12.2,9.8 \mathrm{~Hz}, 1 \mathrm{H}$, one of $\left.\mathrm{C} 1-\mathrm{H}_{2}\right), 1.31\left(\mathrm{~s}, 3 \mathrm{H}, \mathrm{C} 4-\mathrm{CH}_{3}\right), 1.46\left(\mathrm{~m}, 1 \mathrm{H}\right.$, one of $\left.\mathrm{C} 1-\mathrm{H}_{2}\right), 1.49(\mathrm{~m}, 1 \mathrm{H}$, one of $\left.\mathrm{C} 3-\mathrm{H}_{2}\right), 1.52\left(\mathrm{~m}, 1 \mathrm{H}\right.$, one of $\left.\mathrm{C} 2-\mathrm{H}_{2}\right), 1.72\left(\mathrm{ddd}, J=6.3,11.9,16.4 \mathrm{~Hz}, 1 \mathrm{H}\right.$, one of $\left.\mathrm{C} 7-\mathrm{H}_{2}\right), 1.78(\mathrm{~m}, 1 \mathrm{H}$, one of $\left.\mathrm{C} 2-\mathrm{H}_{2}\right), 1.95\left(\mathrm{ddd}, \mathrm{J}=4.4,11.4,13.8 \mathrm{~Hz}, 1 \mathrm{H}\right.$, one of $\left.\mathrm{C} 11-\mathrm{H}_{2}\right), 2.12\left(\mathrm{~m}, 1 \mathrm{H}\right.$, one of $\left.\mathrm{C} 3-\mathrm{H}_{2}\right), 2.18$ $(\mathrm{ddq}, J=6.3,11.9,6.5 \mathrm{~Hz}, 1 \mathrm{H}, \mathrm{C} 8-H), 2.19(\mathrm{~d}, J=4.5 \mathrm{~Hz}, 1 \mathrm{H}, \mathrm{C} 5-\mathrm{H}), 2.22(\mathrm{ddd}, J=8.9,11.5,13.8 \mathrm{~Hz}, 1 \mathrm{H}$, one of $\left.\mathrm{C} 11-\mathrm{H}_{2}\right), 2.28\left(\mathrm{dd}, J=6.3,16.4 \mathrm{~Hz}, 1 \mathrm{H}\right.$, one of $\left.\mathrm{C} 7-\mathrm{H}_{2}\right), 2.54(\mathrm{ddd}, J=4.4,11.5,18.8 \mathrm{~Hz}, 1 \mathrm{H}$, one of $\left.\mathrm{C} 12-\mathrm{H}_{2}\right), 2.62\left(\mathrm{ddd}, J=8.9,11.4,18.8 \mathrm{~Hz}, 1 \mathrm{H}\right.$, one of $\left.\mathrm{C} 12-\mathrm{H}_{2}\right), 4.75(\mathrm{dd}, J=4.5,6.3 \mathrm{~Hz}, 1 \mathrm{H}, \mathrm{C} 6-\mathrm{H})$; ${ }^{13} \mathrm{C}-\mathrm{NMR}\left(125.7 \mathrm{MHz}, \mathrm{CDCl}_{3}\right) \delta 15.4\left(\mathrm{CH}_{3}\right), 17.7\left(\mathrm{CH}_{2}\right), 22.2\left(\mathrm{CH}_{3}\right), 22.4\left(\mathrm{CH}_{3}\right), 24.5\left(\mathrm{CH}_{2}\right), 27.7\left(\mathrm{CH}_{2}\right)$, $28.2\left(\mathrm{CH}_{2}\right), 29.3\left(\mathrm{CH}_{2}\right), 31.2\left(\mathrm{CH}_{2}\right), 32.3(\mathrm{CH}), 38.6(\mathrm{C}), 44.0(\mathrm{C}), 45.3(\mathrm{CH}), 75.6(\mathrm{CH}), 91.2(\mathrm{C}), 176.9(\mathrm{C})$, 183.3 (C); HRMS (ESI) $m / z[\mathrm{M}+\mathrm{Na}]^{+}$calcd for $\mathrm{C}_{17} \mathrm{H}_{24} \mathrm{O}_{4} \mathrm{Na}$ 315.1567; found 315.1566.

(1S,4R,6R,7R,8S,12R)-7-[4-(tert-Butyldimethylsilyl)oxybutyl]-7-hydroxy-1,6,8-trimethyl-2-oxo-3-oxatricyclo [6.3.1. $0^{4,12}$ ]dodecane (45). To an ice-cooled $\left(0{ }^{\circ} \mathrm{C}\right)$ solution of 3-(tert-butyldimethylsilyl)oxypropyl magnesium bromide (44) [prepared from (3-bromopropoxy)(tert-butyl)dimethylsilane (2.00 g, 7.90 mmol) and magnesium tuning (241 mg, $9.93 \mathrm{mmol})]$ in THF $(14 \mathrm{~mL})$ was added $\mathrm{CuBr} \cdot \mathrm{SMe}_{2}(129 \mathrm{mg}$, $0.627 \mathrm{mmol})$, followed by addition of a solution of epoxide $22(39.1 \mathrm{mg}, 0.156 \mathrm{mmol})$ in THF $(0.20 \mathrm{~mL}$ plus $2 \times 0.20 \mathrm{~mL}$ rinse). After $5 \mathrm{~h}$ of stirring at room temperature, the reaction was quenched with saturated aqueous $\mathrm{NH}_{4} \mathrm{Cl}(15 \mathrm{~mL})$, and the resulting mixture was extracted with AcOEt $(3 \times 20 \mathrm{~mL})$. The combined organic extracts were washed with brine $(20 \mathrm{~mL})$, and dried over anhydrous $\mathrm{Na}_{2} \mathrm{SO}_{4}$. Filtration and evaporation in vacuo furnished the pale yellow oil $(869 \mathrm{mg})$, which was purified by column chromatography (silica gel $20 \mathrm{~g}, 10: 1 \rightarrow 6: 1 \rightarrow 3: 1 n$-hexane/AcOEt) to give TBS ether 45 (42.1 $\mathrm{mg}, 48 \%)$ as a pale yellow amorphous. $R_{f} 0.46\left(3: 1 n\right.$-hexane/AcOEt); $[\alpha]_{D}^{22}+23.2\left(c 1.00, \mathrm{CHCl}_{3}\right) ; \mathrm{IR}$ (neat) 3534, 2953, 2927, 2859, 1755, 1471, 1462, 1336, 1253, 1197, 1149, 1099, $994 \mathrm{~cm}^{-1}$; ${ }^{1} \mathrm{H}-\mathrm{NMR}$ (500 $\left.\mathrm{MHz}, \mathrm{C}_{6} \mathrm{D}_{6}\right) \delta 0.08\left(\mathrm{~s}, 6 \mathrm{H}, \mathrm{Si}\left(\mathrm{CH}_{3}\right)_{2}\right), 0.65\left(\mathrm{~d}, J=6.6 \mathrm{~Hz}, 3 \mathrm{H}, \mathrm{C} 8-\mathrm{CH}_{3}\right), 0.78(\mathrm{br} \mathrm{s}, 1 \mathrm{H}, \mathrm{OH}), 0.96(\mathrm{~m}$, $1 \mathrm{H}$, one of $\left.\mathrm{C} 1-\mathrm{H}_{2}\right), 0.97\left(\mathrm{~s}, 6 \mathrm{H}, \mathrm{C} 4-\mathrm{CH}_{3}, \mathrm{C} 10-\mathrm{CH}_{3}\right), 1.00\left(\mathrm{~s}, 9 \mathrm{H}, \mathrm{SiC}\left(\mathrm{CH}_{3}\right)_{3}\right), 1.14-1.30(\mathrm{~m}, 6 \mathrm{H}$, one of $\mathrm{C} 2-\mathrm{H}_{2}$, one of $\left.\mathrm{C} 3-\mathrm{H}_{2}, \mathrm{C} 11-\mathrm{H}_{2}, \mathrm{C} 12-\mathrm{H}_{2}\right), 1.35-1.45\left(\mathrm{~m}, 4 \mathrm{H}\right.$, one of $\mathrm{C} 2-\mathrm{H}_{2}$, one of $\left.\mathrm{C} 7-\mathrm{H}_{2}, \mathrm{C} 13-\mathrm{H}_{2}\right), 1.47$ $\left(\mathrm{m}, 1 \mathrm{H}\right.$, one of $\left.\mathrm{C} 1-\mathrm{H}_{2}\right), 1.77(\mathrm{ddq}, J=6.2,11.2,6.6 \mathrm{~Hz}, 1 \mathrm{H}, \mathrm{C} 8-\mathrm{H}), 1.92(\mathrm{dd}, J=6.2,15.8 \mathrm{~Hz}, 1 \mathrm{H}$, one of C7- $\left.\mathrm{H}_{2}\right), 2.00(\mathrm{~d}, J=4.6 \mathrm{~Hz}, 1 \mathrm{H}, \mathrm{C} 5-\mathrm{H}), 2.18\left(\mathrm{dt}, J=4.3,13.7 \mathrm{~Hz}, 1 \mathrm{H}\right.$, one of $\left.\mathrm{C}^{-}-\mathrm{H}_{2}\right), 3.52(\mathrm{t}, J=6.1$ $\left.\mathrm{Hz}, 2 \mathrm{H}, \mathrm{C} 14-\mathrm{H}_{2}\right), 4.36(\mathrm{dd}, J=4.6,6.4 \mathrm{~Hz}, 1 \mathrm{H}, \mathrm{C} 6-\mathrm{H}) ;{ }^{13} \mathrm{C}-\mathrm{NMR}\left(125.7 \mathrm{MHz}, \mathrm{C}_{6} \mathrm{D}_{6}\right) \delta-5.1\left(\mathrm{CH}_{3}\right), 16.6$ $\left(\mathrm{CH}_{3}\right), 18.51(\mathrm{C}), 18.55\left(\mathrm{CH}_{2}\right), 21.9\left(\mathrm{CH}_{2}\right), 22.6\left(\mathrm{CH}_{3}\right), 22.9\left(\mathrm{CH}_{3}\right), 26.1\left(\mathrm{CH}_{3}\right), 28.8\left(\mathrm{CH}_{2}\right), 28.9\left(\mathrm{CH}_{2}\right)$, $32.0\left(\mathrm{CH}_{2}\right), 32.3(\mathrm{CH}), 34.0\left(\mathrm{CH}_{2}\right), 34.8\left(\mathrm{CH}_{2}\right), 39.8(\mathrm{C}), 43.7(\mathrm{C}), 44.9(\mathrm{CH}), 62.8\left(\mathrm{CH}_{2}\right), 75.48(\mathrm{C}), 75.52$ (C), 182.6 (C); HRMS (ESI) $m / z$ [M + Na] $]^{+}$calcd for $\mathrm{C}_{24} \mathrm{H}_{44} \mathrm{O}_{4} \mathrm{SiNa}$ 447.2907; found 447.2891.

(1S,4R,6R,7R,8S,12R)-7-Hydroxy-7-(4-hydroxybutyl)-1,6,8-trimethyl-2-oxo-3-oxatricyclo[6.3.1.0 ${ }^{4,12}$ ]dodecane (46). $\mathrm{Bu}_{4} \mathrm{NF}$ in THF $(1.0 \mathrm{M}, 0.38 \mathrm{~mL}, 0.38 \mathrm{mmol})$ was added to an ice-cooled $\left(0{ }^{\circ} \mathrm{C}\right)$ solution of TBS ether $45(32.1 \mathrm{mg}, 0.76 \mathrm{mmol})$ in THF $(1.0 \mathrm{~mL})$. After $1 \mathrm{~h}$ of stirring at room temperature, the mixture 
was partitioned between AcOEt $(2 \mathrm{~mL})$ and $\mathrm{H}_{2} \mathrm{O}(2 \mathrm{~mL})$, and the aqueous layer was extracted with AcOEt $(3 \times 2 \mathrm{~mL})$. The combined organic extracts were washed with brine $(5 \mathrm{~mL})$, and dried over anhydrous $\mathrm{Na}_{2} \mathrm{SO}_{4}$. Filtration and evaporation in vacuo furnished the pale yellow oil (144 mg), which was purified by column chromatography (silica gel $2 \mathrm{~g}, 1: 4 n$-hexane/AcOEt) to give diol 46 (19.3 mg, $82 \%)$ as a colorless amorphous. $R_{f} 0.33\left(1: 2 n\right.$-hexane/AcOEt); $[\alpha]_{D}^{22}+24.4\left(c 0.90, \mathrm{CHCl}_{3}\right)$; IR (neat) 3478, 2951, 2930, 2870, 1747, 1462, 1454, 1350, 1263, 1199, 1149, 1099, 1072, 1041, $991 \mathrm{~cm}^{-1} ;{ }^{1} \mathrm{H}-\mathrm{NMR}$ $\left(500 \mathrm{MHz}, \mathrm{C}_{6} \mathrm{D}_{6}\right) \delta 0.54(\mathrm{~s}, 1 \mathrm{H}, \mathrm{OH}), 0.61\left(\mathrm{~d}, J=6.7 \mathrm{~Hz}, 3 \mathrm{H}, \mathrm{C} 8-\mathrm{CH}_{3}\right), 0.86(\mathrm{~s}, 1 \mathrm{H}, \mathrm{OH}), 0.91(\mathrm{~m}, 1 \mathrm{H}$, one of $\left.\mathrm{C} 1-\mathrm{H}_{2}\right), 0.95\left(\mathrm{~s}, 3 \mathrm{H}, \mathrm{C} 10-\mathrm{CH}_{3}\right), 0.97\left(\mathrm{~s}, 3 \mathrm{H}, \mathrm{C} 4-\mathrm{CH}_{3}\right), 1.07-1.30\left(\mathrm{~m}, 8 \mathrm{H}\right.$, one of C1- $\mathrm{H}_{2}$, one of C2- $\mathrm{H}_{2}$, $\left.\mathrm{C} 11-\mathrm{H}_{2}, \mathrm{C} 12-\mathrm{H}_{2}, \mathrm{C} 13-\mathrm{H}_{2}\right), 1.36-1.48\left(\mathrm{~m}, 3 \mathrm{H}\right.$, one of $\mathrm{C} 2-\mathrm{H}_{2}$, one of $\mathrm{C} 3-\mathrm{H}_{2}$, one of $\left.\mathrm{C} 7-\mathrm{H}_{2}\right), 1.73(\mathrm{ddq}, J=$ 6.2, 11.3, $6.7 \mathrm{~Hz}, 1 \mathrm{H}, \mathrm{C} 8-\mathrm{H}), 1.91\left(\mathrm{ddd}, J=1.3,6.2,15.9 \mathrm{~Hz}, 1 \mathrm{H}\right.$, one of $\left.\mathrm{C} 7-\mathrm{H}_{2}\right), 2.00(\mathrm{~d}, J=4.8 \mathrm{~Hz}, 1 \mathrm{H}$, $\mathrm{C} 5-H), 2.18\left(\mathrm{dt}, J=4.2,13.8 \mathrm{~Hz}, 1 \mathrm{H}\right.$, one of $\left.\mathrm{C} 3-H_{2}\right), 3.30\left(\mathrm{t}, J=6.1 \mathrm{~Hz}, 2 \mathrm{H}, \mathrm{C} 14-\mathrm{H}_{2}\right), 4.35(\mathrm{ddd}, J=1.3$, 4.8, 6.2 Hz, 1H, C6- $H) ;{ }^{13} \mathrm{C}-\mathrm{NMR}\left(125.7 \mathrm{MHz}, \mathrm{C}_{6} \mathrm{D}_{6}\right) \delta 16.6\left(\mathrm{CH}_{3}\right), 18.6\left(\mathrm{CH}_{2}\right), 21.8\left(\mathrm{CH}_{2}\right), 22.5\left(\mathrm{CH}_{3}\right)$, $22.9\left(\mathrm{CH}_{3}\right), 28.7\left(\mathrm{CH}_{2}\right), 28.9\left(\mathrm{CH}_{2}\right), 32.0\left(\mathrm{CH}_{2}\right), 32.3(\mathrm{CH}), 33.7\left(\mathrm{CH}_{2}\right), 34.7\left(\mathrm{CH}_{2}\right), 39.8(\mathrm{C}), 43.7(\mathrm{C}), 44.9$ $(\mathrm{CH}), 62.2\left(\mathrm{CH}_{2}\right), 75.4(\mathrm{C}), 75.6(\mathrm{CH}), 182.7(\mathrm{C})$; HRMS (ESI) $m / z[\mathrm{M}+\mathrm{Na}]^{+}$calcd for $\mathrm{C}_{18} \mathrm{H}_{30} \mathrm{O}_{4} \mathrm{Na}$ 333.2042; found 333.2052.

Marrulactone (14). TEMPO (2.3 mg, $14.7 \mu \mathrm{mol})$ was added to an ice-cooled $\left(0{ }^{\circ} \mathrm{C}\right)$ mixture of diol 46 $(13.1 \mathrm{mg}, 42.2 \mu \mathrm{mol})$ and $\mathrm{PhI}(\mathrm{OAc})_{2}(40.5 \mathrm{mg}, 0.125 \mathrm{mmol})$ in $\mathrm{CH}_{2} \mathrm{Cl}_{2}(0.3 \mathrm{~mL})$. After $4 \mathrm{~h}$ of stirring at room temperature, the reaction was quenched with $1 \mathrm{M}$ aqueous $\mathrm{Na}_{2} \mathrm{~S}_{2} \mathrm{O}_{3}(1 \mathrm{~mL})$, and the resulting mixture was extracted with $\operatorname{AcOEt}(3 \times 1 \mathrm{~mL})$. The combined organic extracts were washed with brine $(3 \mathrm{~mL})$, and dried over anhydrous $\mathrm{Na}_{2} \mathrm{SO}_{4}$. Filtration and evaporation in vacuo furnished the colorless oil (64.0 mg), which was purified by column chromatography (silica gel $1 \mathrm{~g}, 1: 1 \mathrm{n}$-hexane/AcOEt) to give marrulactone $(\mathbf{1 4}, 11.8 \mathrm{mg}, 91 \%)$ as a colorless amorphous. $R_{f} 0.60\left(1: 2 n\right.$-hexane/AcOEt); $[\alpha]_{D}^{22}$ -11.6 (c 0.61, $\mathrm{CHCl}_{3}$ ) [lit. [12], [ $\left.\alpha\right]_{D}^{25}-23.80$ (c 0.22, $\mathrm{CHCl}_{3}$ )]; IR (neat) 3017, 2961, 2928, 2872, 1769, 1719, 1458, 1391, 1329, 1261, 1244, 1215, 1188, 1117, 1070, 1030, $1003 \mathrm{~cm}^{-1} ;{ }^{1} \mathrm{H}-\mathrm{NMR}\left(500 \mathrm{MHz}, \mathrm{CDCl}_{3}\right) \delta 0.98$ $\left(\mathrm{d}, \mathrm{J}=6.3 \mathrm{~Hz}, 3 \mathrm{H}, \mathrm{C} 8-\mathrm{CH}_{3}\right), 1.11\left(\mathrm{~s}, 3 \mathrm{H}, \mathrm{C} 10-\mathrm{CH}_{3}\right), 1.31\left(\mathrm{~s}, 3 \mathrm{H}, \mathrm{C} 4-\mathrm{CH}_{3}\right), 1.34-1.42\left(\mathrm{~m}, 2 \mathrm{H}, \mathrm{C} 1-\mathrm{H}_{2}\right), 1.48$ $\left(\mathrm{m}, 1 \mathrm{H}\right.$, one of $\left.\mathrm{C} 3-\mathrm{H}_{2}\right), 1.55\left(\mathrm{~m}, 1 \mathrm{H}\right.$, one of $\left.\mathrm{C} 2-\mathrm{H}_{2}\right), 1.74-1.80\left(\mathrm{~m}, 2 \mathrm{H}\right.$, one of $\mathrm{C} 2-\mathrm{H}_{2}$, one of $\left.\mathrm{C} 11-\mathrm{H}_{2}\right)$, 1.80-1.94 (m, 4H, one of C7- $\mathrm{H}_{2}$, one of $\left.\mathrm{C} 11-\mathrm{H}_{2}, \mathrm{C} 12-\mathrm{H}_{2}\right), 2.09-2.20\left(\mathrm{~m}, 3 \mathrm{H}\right.$, one of $\mathrm{C} 3-\mathrm{H}_{2}$, one of C7- $\mathrm{H}_{2}$, C8-H), $2.25\left(\mathrm{ddd}, J=6.2,11.2,17.3 \mathrm{~Hz}, 1 \mathrm{H}\right.$, one of C13- $\left.\mathrm{H}_{2}\right), 2.32(\mathrm{~d}, J=4.4 \mathrm{~Hz}, 1 \mathrm{H}, \mathrm{C} 5-H), 2.57(\mathrm{ddt}, J=$ 2.0, 17.3, $4.1 \mathrm{~Hz}, 1 \mathrm{H}$, one of $\left.\mathrm{C} 13-\mathrm{H}_{2}\right), 4.78(\mathrm{ddd}, J=0.7,4.4,5.5 \mathrm{~Hz}, 1 \mathrm{H}, \mathrm{C} 6-\mathrm{H}) ;{ }^{13} \mathrm{C}-\mathrm{NMR}(125.7 \mathrm{MHz}$, $\left.\mathrm{CDCl}_{3}\right) \delta 16.5\left(\mathrm{CH}_{3}\right), 17.8\left(\mathrm{CH}_{2}\right), 18.9\left(\mathrm{CH}_{2}\right), 22.0\left(\mathrm{CH}_{3}\right), 22.8\left(\mathrm{CH}_{3}\right), 26.1\left(\mathrm{CH}_{2}\right), 28.1\left(\mathrm{CH}_{2}\right), 28.6\left(\mathrm{CH}_{2}\right)$, $30.4\left(\mathrm{CH}_{2}\right), 30.9\left(\mathrm{CH}_{2}\right), 34.3(\mathrm{CH}), 40.8(\mathrm{C}), 44.0(\mathrm{C}), 44.3(\mathrm{CH}), 75.8(\mathrm{CH}), 88.3(\mathrm{C}), 172.2(\mathrm{C}), 183.5(\mathrm{C})$; HRMS (ESI) $m / z[\mathrm{M}+\mathrm{Na}]^{+}$calcd for $\mathrm{C}_{18} \mathrm{H}_{26} \mathrm{O}_{4} \mathrm{Na} 329.1723$; found 329.1711.

Marrulanic acid (10). $\mathrm{LiOH}(18.7 \mathrm{mg}, 0.78 \mathrm{mmol})$ was added to a solution of marrulactone $(\mathbf{1 4}, 11.8 \mathrm{mg}$, $38.5 \mu \mathrm{mol})$ in $\mathrm{THF} / \mathrm{H}_{2} \mathrm{O}(2: 1,1.2 \mathrm{~mL})$. After $31 \mathrm{~h}$ of stirring, the mixture was acidified with $1 \mathrm{M}$ aqueous $\mathrm{HCl}(3 \mathrm{~mL})$, and the resulting mixture was extracted with $\mathrm{AcOEt}(3 \times 3 \mathrm{~mL})$. The combined organic extracts were washed with brine $(5 \mathrm{~mL})$, and dried over anhydrous $\mathrm{Na}_{2} \mathrm{SO}_{4}$. Filtration and evaporation in vacuo furnished the colorless oil $(11.7 \mathrm{mg})$, which was purified by column chromatography (silica gel $1 \mathrm{~g}, 1: 1 n$-hexane/AcOEt $\rightarrow$ AcOEt $\rightarrow$ 20:1 AcOEt/MeOH) to give marrulanic acid (10, 10.5 mg, 84\%) as a colorless amorphous. $R_{f} 0.19\left(1: 2 n\right.$-hexane/AcOEt); $[\alpha]_{D}^{27}+25.4\left(c 0.53, \mathrm{CHCl}_{3}\right)\left[\right.$ lit. [12], $[\alpha]_{D}^{25}-10.8$ (c 1.2, $\mathrm{CHCl}_{3}$ )]; IR (neat) 3536, 3196, 2949, 2928, 2868, 1761, 1732, 1464, 1412, 1391, 1287, 1260, 1196, 1179, 1159, 1126, 1094, 1078, 1042, $1018 \mathrm{~cm}^{-1} ;{ }^{1} \mathrm{H}-\mathrm{NMR}\left(500 \mathrm{MHz}, \mathrm{CDCl}_{3}\right) \delta 0.92(\mathrm{~d}, J=6.5 \mathrm{~Hz}, 3 \mathrm{H}$, $\left.\mathrm{C}_{8}-\mathrm{CH}_{3}\right), 1.04\left(\mathrm{~s}, 3 \mathrm{H}, \mathrm{C} 10-\mathrm{CH}_{3}\right), 1.26\left(\mathrm{~m}, 1 \mathrm{H}\right.$, one of $\left.\mathrm{C} 1-\mathrm{H}_{2}\right), 1.29\left(\mathrm{~s}, 3 \mathrm{H}, \mathrm{C} 4-\mathrm{CH}_{3}\right), 1.44-1.80(\mathrm{~m}, 9 \mathrm{H}$, one of $\mathrm{C} 1-\mathrm{H}_{2}, \mathrm{C} 2-\mathrm{H}_{2}$, one of $\mathrm{C} 3-\mathrm{H}_{2}$, one of $\left.\mathrm{C} 7-\mathrm{H}_{2}, \mathrm{C} 11-\mathrm{H}_{2}, \mathrm{C} 12-\mathrm{H}_{2}\right), 2.04-2.17\left(\mathrm{~m}, 3 \mathrm{H}\right.$, one of C3- $\mathrm{H}_{2}$, one of $\left.\mathrm{C} 7-H_{2}, \mathrm{C} 8-H\right), 2.24(\mathrm{~d}, J=4.6 \mathrm{~Hz}, 1 \mathrm{H}, \mathrm{C} 5-H), 2.38\left(\mathrm{dt}, J=1.8,7.0 \mathrm{~Hz}, 2 \mathrm{H}, \mathrm{C} 13-\mathrm{H}_{2}\right), 4.74(\mathrm{ddd}, J=1.2$, 4.6, $5.8 \mathrm{~Hz}, 1 \mathrm{H}, \mathrm{C} 6-\mathrm{H}) ;{ }^{13} \mathrm{C}-\mathrm{NMR}\left(125.0 \mathrm{MHz}, \mathrm{CDCl}_{3}\right) \delta 16.5\left(\mathrm{CH}_{3}\right), 18.2\left(\mathrm{CH}_{2}\right), 20.3\left(\mathrm{CH}_{2}\right), 22.3\left(\mathrm{CH}_{3}\right)$, $22.9\left(\mathrm{CH}_{3}\right), 28.3\left(\mathrm{CH}_{2}\right), 28.6\left(\mathrm{CH}_{2}\right), 31.5\left(\mathrm{CH}_{2}\right), 32.2(\mathrm{CH}), 34.29\left(\mathrm{CH}_{2}\right), 34.31\left(\mathrm{CH}_{2}\right), 39.7(\mathrm{C}), 43.8(\mathrm{C})$, $44.8(\mathrm{CH}), 75.6(\mathrm{C}), 76.2(\mathrm{CH}), 178.5(\mathrm{C}), 183.9(\mathrm{C})$; HRMS (ESI) $m / z[\mathrm{M}+\mathrm{Na}]^{+}$calcd for $\mathrm{C}_{18} \mathrm{H}_{28} \mathrm{O}_{5} \mathrm{Na}$ 347.1834; found 347.1813 . 
[1S,4R,6R,7R,7(2S,3S,4R,5R),8S,12R]-7-[2-(3,4-Dihydroxy-2,5-dimethoxytetrahydrofuran-3-yl)ethyl]-7-hydro $x y$-1,6,8-trimethyl-2-oxo-3-oxatricyclo[6.3.1.0 $\left.{ }^{4,12}\right]$ dodecane (40). $\mathrm{TsOH}(0.4 \mathrm{mg}, 2.3 \mu \mathrm{mol})$ was added to a solution of marrulibacetal A $(\mathbf{1 7}, 0.90 \mathrm{mg}, 2.3 \mu \mathrm{mol})$ in $\mathrm{MeOH}(1.0 \mathrm{~mL})$, and the mixture was heated at reflux for $5 \mathrm{~h}$. After cooling to $0{ }^{\circ} \mathrm{C}$, the reaction was quenched with half saturated aqueous $\mathrm{NaHCO}_{3}$ $(4 \mathrm{~mL})$, and the resulting mixture was extracted with AcOEt $(2 \times 5 \mathrm{~mL})$. The combined organic extracts were washed with brine $(5 \mathrm{~mL})$, and dried over anhydrous $\mathrm{Na}_{2} \mathrm{SO}_{4}$. Filtration and evaporation in vacuo furnished the colorless oil (1.4 mg), which was purified by flash column chromatography (silica gel $0.8 \mathrm{~g}, 1: 1 \mathrm{n}$-hexane/AcOEt $\rightarrow$ AcOEt $)$ to give triol $40(0.24 \mathrm{mg}, 25 \%)$ as a colorless oil. $R_{f} 0.30(1: 1$ $n$-hexane/AcOEt); $[\alpha]_{D}^{23}+16.5$ (c 0.46, benzene); IR (neat) 3447, 2930, 1744, 1466, 1449, 1389, 1288, 1198, 1130, 1094, 1042, $991 \mathrm{~cm}^{-1} ;{ }^{1} \mathrm{H}-\mathrm{NMR}\left(500 \mathrm{MHz}, \mathrm{CDCl}_{3}\right) \delta 0.91\left(\mathrm{~d}, J=6.4 \mathrm{~Hz}, 3 \mathrm{H}, \mathrm{C} 8-\mathrm{CH}_{3}\right), 1.05(\mathrm{~s}, 3 \mathrm{H}$, $\left.\mathrm{C} 10-\mathrm{CH}_{3}\right), 1.28\left(\mathrm{~m}, 1 \mathrm{H}\right.$, one of $\left.\mathrm{C} 1-\mathrm{H}_{2}\right), 1.29\left(\mathrm{~s}, 3 \mathrm{H}, \mathrm{C} 4-\mathrm{CH}_{3}\right), 1.45\left(\mathrm{~m}, 1 \mathrm{H}\right.$, one of $\left.\mathrm{C} 3-\mathrm{H}_{2}\right), 1.51(\mathrm{~m}, 1 \mathrm{H}$, one of $\left.\mathrm{C} 2-\mathrm{H}_{2}\right), 1.65\left(\mathrm{~m}, 1 \mathrm{H}\right.$, one of $\left.\mathrm{C} 7-\mathrm{H}_{2}\right), 1.74\left(\mathrm{~m}, 1 \mathrm{H}\right.$, one of $\left.\mathrm{C} 2-\mathrm{H}_{2}\right), 1.60-1.98\left(\mathrm{~m}, 5 \mathrm{H}\right.$, one of $\mathrm{C} 1-\mathrm{H}_{2}$, $\left.\mathrm{C} 11-\mathrm{H}_{2}, \mathrm{C} 12-\mathrm{H}_{2}\right), 2.07(\mathrm{~m}, 1 \mathrm{H}, \mathrm{C} 8-\mathrm{H}), 2.11\left(\mathrm{~m}, 1 \mathrm{H}\right.$, one of $\left.\mathrm{C} 3-\mathrm{H}_{2}\right), 2.14\left(\mathrm{~m}, 1 \mathrm{H}\right.$, one of $\left.\mathrm{C} 7-\mathrm{H}_{2}\right), 2.22(\mathrm{~d}, \mathrm{~J}$ $=4.5 \mathrm{~Hz}, 1 \mathrm{H}, \mathrm{C} 5-\mathrm{H}), 3.40\left(\mathrm{~s}, 3 \mathrm{H}, \mathrm{C} 16-\mathrm{OCH}_{3}\right), 3.48\left(\mathrm{~s}, 3 \mathrm{H}, \mathrm{C} 15-\mathrm{OCH}_{3}\right), 3.92(\mathrm{~d}, J=3.7 \mathrm{~Hz}, 1 \mathrm{H}, \mathrm{C} 14-\mathrm{H})$, $4.73(\mathrm{dd}, J=4.5,6.5 \mathrm{~Hz}, 1 \mathrm{H}, \mathrm{C} 6-\mathrm{H}), 4.77(\mathrm{~s}, 1 \mathrm{H}, \mathrm{C} 16-\mathrm{H}), 4.89$ (d, J = 3.7 Hz, 1H, C15-H); ${ }^{13} \mathrm{C}-\mathrm{NMR}$ $\left(125.7 \mathrm{MHz}, \mathrm{CDCl}_{3}\right) \delta 16.4\left(\mathrm{CH}_{3}\right), 18.1\left(\mathrm{CH}_{2}\right), 22.3\left(\mathrm{CH}_{3}\right), 22.9\left(\mathrm{CH}_{3}\right), 28.0\left(\mathrm{CH}_{2}\right), 28.26\left(\mathrm{CH}_{2}\right), 28.28$ $\left(\mathrm{CH}_{2}\right), 28.4\left(\mathrm{CH}_{2}\right), 31.5\left(\mathrm{CH}_{2}\right), 32.6(\mathrm{CH}), 40.0(\mathrm{C}), 43.9(\mathrm{C}), 44.9(\mathrm{CH}), 55.1\left(\mathrm{CH}_{3}\right), 56.4\left(\mathrm{CH}_{3}\right), 75.4(\mathrm{C})$, $76.2(\mathrm{CH}), 80.3(\mathrm{CH}), 81.4(\mathrm{C}), 108.3(\mathrm{CH}), 110.7(\mathrm{CH}), 184.0(\mathrm{C})$; HRMS (ESI) $\mathrm{m} / \mathrm{z}[\mathrm{M}+\mathrm{Na}]^{+}$calcd for $\mathrm{C}_{22} \mathrm{H}_{36} \mathrm{O}_{8} \mathrm{Na}$ 451.2302; found 451.2308.

Desertine (18). TsOH (1.6 mg, $9.3 \mu \mathrm{mol})$ was added to a solution of bisacetal $42(2.0 \mathrm{mg}, 4.7 \mu \mathrm{mol})$ in $\mathrm{MeOH}(0.5 \mathrm{~mL})$, and the mixture was heated at reflux for $3.5 \mathrm{~h}$. After cooling to $0{ }^{\circ} \mathrm{C}$, the reaction was quenched with saturated aqueous $\mathrm{NaHCO}_{3}(2 \mathrm{~mL})$, and the resulting mixture was extracted with AcOEt $(2 \times 5 \mathrm{~mL})$. The combined organic extracts were washed with brine $(10 \mathrm{~mL})$, and dried over anhydrous $\mathrm{Na}_{2} \mathrm{SO}_{4}$. Filtration and evaporation in vacuo furnished the pale yellow oil ( $\left.3.6 \mathrm{mg}\right)$, which was purified by flash column chromatography (silica gel $0.8 \mathrm{~g}, 1: 1 n$-hexane/AcOEt) to give desertine $(\mathbf{1 8}, 0.34 \mathrm{mg}, 16 \%)$ as a colorless oil. $R_{f} 0.30\left(1: 1 n\right.$-hexane/AcOEt); $[\alpha]_{D}^{23}+22.8(c 0.50$, benzene $)$ (lit. [14], [ $\alpha]_{D}^{25}$-56.6); IR (neat) 3464, 2930, 1748, 1463, 1450, 1391, 1256, 1198, 1152, 1099, 1043, $989 \mathrm{~cm}^{-1}$; ${ }^{1} \mathrm{H}-\mathrm{NMR}\left(500 \mathrm{MHz}, \mathrm{CDCl}_{3}\right) \delta 0.91\left(\mathrm{~d}, J=6.2 \mathrm{~Hz}, 3 \mathrm{H}, \mathrm{C} 8-\mathrm{CH}_{3}\right), 1.04\left(\mathrm{~s}, 3 \mathrm{H}, \mathrm{C} 10-\mathrm{CH}_{3}\right), 1.28(\mathrm{~m}, 1 \mathrm{H}$, one of $\left.\mathrm{C} 1-\mathrm{H}_{2}\right), 1.29\left(\mathrm{~s}, 3 \mathrm{H}, \mathrm{C} 4-\mathrm{CH}_{3}\right), 1.45\left(\mathrm{~m}, 1 \mathrm{H}\right.$, one of $\left.\mathrm{C} 3-\mathrm{H}_{2}\right), 1.51\left(\mathrm{~m}, 1 \mathrm{H}\right.$, one of $\left.\mathrm{C} 2-\mathrm{H}_{2}\right), 1.65(\mathrm{~m}, 1 \mathrm{H}$, one of $\left.\mathrm{C} 7-\mathrm{H}_{2}\right), 1.74\left(\mathrm{~m}, 1 \mathrm{H}\right.$, one of $\left.\mathrm{C} 2-\mathrm{H}_{2}\right), 1.60-1.98\left(\mathrm{~m}, 5 \mathrm{H}\right.$, one of $\left.\mathrm{C} 1-\mathrm{H}_{2}, \mathrm{C} 11-\mathrm{H}_{2}, \mathrm{C} 12-\mathrm{H}_{2}\right), 2.07(\mathrm{~m}$, $1 \mathrm{H}, \mathrm{C} 8-\mathrm{H}), 2.11\left(\mathrm{~m}, 1 \mathrm{H}\right.$, one of C3- $\left.\mathrm{H}_{2}\right), 2.14\left(\mathrm{~m}, 1 \mathrm{H}\right.$, one of C7- $\left.\mathrm{H}_{2}\right), 2.23(\mathrm{~d}, J=4.0 \mathrm{~Hz}, 1 \mathrm{H}, \mathrm{C} 5-\mathrm{H}), 3.41(\mathrm{~s}$, $\left.3 \mathrm{H}, \mathrm{C} 16-\mathrm{OCH}_{3}\right), 3.47\left(\mathrm{~s}, 3 \mathrm{H}, \mathrm{C} 15-\mathrm{OCH}_{3}\right), 3.95(\mathrm{~d}, J=3.5 \mathrm{~Hz}, 1 \mathrm{H}, \mathrm{C} 14-\mathrm{H}), 4.73(\mathrm{dd}, J=4.0,6.9 \mathrm{~Hz}, 1 \mathrm{H}$, C6-H), $4.76(\mathrm{~s}, 1 \mathrm{H}, \mathrm{C} 16-\mathrm{H}), 4.90(\mathrm{~d}, J=3.5 \mathrm{~Hz}, 1 \mathrm{H}, \mathrm{C} 15-\mathrm{H}) ;{ }^{13} \mathrm{C}-\mathrm{NMR}\left(125.7 \mathrm{MHz}, \mathrm{CDCl}_{3}\right) \delta 16.8\left(\mathrm{CH}_{3}\right)$, 18.2 $\left(\mathrm{CH}_{2}\right), 22.2\left(\mathrm{CH}_{3}\right), 23.0\left(\mathrm{CH}_{3}\right), 27.4\left(\mathrm{CH}_{2}\right), 28.3\left(\mathrm{CH}_{2}\right), 28.4\left(\mathrm{CH}_{2}\right), 28.5\left(\mathrm{CH}_{2}\right), 31.6\left(\mathrm{CH}_{2}\right), 32.5(\mathrm{CH})$, $40.1(\mathrm{C}), 43.9(\mathrm{C}), 45.0(\mathrm{CH}), 55.1\left(\mathrm{CH}_{3}\right), 56.4\left(\mathrm{CH}_{3}\right), 75.3(\mathrm{C}), 76.2(\mathrm{CH}), 80.2(\mathrm{CH}), 81.2(\mathrm{C}), 108.6(\mathrm{CH})$, $110.8(\mathrm{CH}), 183.8(\mathrm{C})$; HRMS (ESI) $\mathrm{m} / z[\mathrm{M}+\mathrm{Na}]^{+}$calcd for $\mathrm{C}_{22} \mathrm{H}_{36} \mathrm{O}_{8} \mathrm{Na} 451.2302$; found 451.2313 .

\section{Conclusions}

Total syntheses of eight labdane diterpene lactones, marrubiin (1), marrulanic acid (10), cyllenine C (12), marrulibacetal (13), marrulactone (14), marrulibacetal A (17), desertine (18), and marrubasch F (19), have been accomplished. Since chiral building block 20 could be prepared from (4R,6S)-6-[(tert-butyldimethylsilyl)oxymethyl]-4-methyltetrahydro-2H-pyran-2-one [53] in 37\% yield over eight steps, the syntheses proceeded in 19-22 steps involving a stereoselective intramolecular PKR, and these natural products were obtained in yields ranging from $1.1 \%$ (marrulibacetal A) to $10.9 \%$ (cyllenin C). Stereochemistries of desertine were established by the facts that the compound could be obtained by both osmylation of bisacetal $\mathbf{3 7}$ and transacetalization of the diastereomer of marrulibacetal A with $\mathrm{MeOH}$. The syntheses illustrate the synthetic utility of chiral building block 20 for the synthesis of this class of diterpenoids. The synthetic strategy also features elongation of the $\mathrm{C} 9$ side chain through an epoxide-opening reaction as well as high convergency and flexibility. 
The syntheses of other natural products and non-natural analogues for biological and pharmaceutical investigations are in progress in our laboratory and will be reported in due course.

Supplementary Materials: The following are available online. Comparison data for natural products and synthetic materials, and copies of ${ }^{1} \mathrm{H}$ and ${ }^{13} \mathrm{C}-\mathrm{NMR}$ spectra for all new compounds.

Author Contributions: Y.S. (Yukari Sakagami), N.K., Y.S. (Yuki Sawayama), and H.Y. performed the experiments. S.N. conceived and designed the project. H.Y. and S.N. directed the investigations and prepared this manuscript. All authors discussed the experimental results. All authors have read and agreed to the published version of the manuscript.

Funding: This research was supported in part by the Platform Project for Supporting in Drug Discovery and Life Science Research from Japan Agency for Medical Research and Development (AMED), and Shionogi Award in Synthetic Organic Chemistry, Japan.

Acknowledgments: The authors acknowledge the assistance of the Research Equipment Sharing Center at Nagoya City University. We are grateful to Mr. Kota Suzuki of the Faculty of Pharmaceutical Sciences, Nagoya City University, for experimental assistance.

Conflicts of Interest: The authors declare no conflict of interest.

\section{References and Notes}

1. Meyre-Silva, C.; Cechinel-Filho, V. A Review of the Chemical and Pharmacological Aspects of the Genus Marrubium. Curr. Pharm. Des. 2010, 16, 3503-3518. [CrossRef] [PubMed]

2. Popoola, O.K.; Elbagory, A.M.; Ameer, F.; Hussein, A.A. Marrubiin. Molecules 2013, 18, 9049-9060. [CrossRef] [PubMed]

3. Bardai, S.E.; Morel, N.; Wibo, M.; Fabre, N.; Llabres, G.; Lyoussi, B.; Quetin-Leclercq, J. The Vasorelaxant Activity of Marrubenol and Marrubiin from Marrubium vulgare. Planta Med. 2003, 69, 75-77. [CrossRef] [PubMed]

4. Piozzi, F.; Bruno, M.; Rosselli, S.; Maggio, A. The Diterpenoids of the Genus Marrubium (Lamiaceae). Nat. Prod. Commun. 2006, 1, 585-592. [CrossRef]

5. Salei, L.A.; Popa, D.P.; Lazur'evskii, G.V. Diterpenoids from Marrubium peregrinum. Khim. Prir. Soedin. 1966, $2,249-251$.

6. Iida, A.; Tanaka, Y.; Mihara, T.; Tabata, M.; Honda, G.; Shingu, T.; Takeda, Y.; Takaishi, Y.; Yesilada, E.; Sezik, E.; et al. Marrubinones A and B, New Labdane Diterpenoids from Marrubium astracanicum (Labiatae). Chem. Pharm. Bull. 1995, 43, 1454-1457. [CrossRef]

7. Hatam, N.A.R.; Porzel, A.; Seifert, K. Polyodonine, a prefuranic labdane diterpene from Marrubium polydon. Phytochemistry 1995, 40, 1575-1576. [CrossRef]

8. Karioti, A.; Heilmann, J.; Skaltsa, H. Labdane Diterpenes from Marrubium velutinum and Marrubium cylleneum. Phytochemistry 2005, 66, 1060-1066. [CrossRef]

9. Rigano, D.; Grassia, A.; Borrelli, F.; Aviello, G.; Piozzi, F.; Bruno, M.; Apostolides Arnold, N.; Capasso, R.; Senatore, F. Phytochemical and Pharmacological Studies on the Acetonic Extract of Marrubium globosum ssp. libanoticum. Planta Med. 2006, 72, 575-578. [CrossRef]

10. Rigano, D.; Grassia, A.; Bruno, M.; Rosselli, S.; Piozzi, F.; Formisano, C.; Apostolides Arnold, N.; Senatore, F. Labdane Diterpenoids from Marrubium globosum ssp. libanoticum. J. Nat. Prod. 2006, 69, 836-838. [CrossRef]

11. Karioti, A.; Skopeliti, M.; Tsitsilonis, O.; Heilmann, J.; Skaltsa, H. Cytotoxicity and Immunomodulating Characteristics of Labdane Diterpenes from Marrubium cylleneum and Marrubium velutinum. Phytochemistry 2007, 68, 1587-1594. [CrossRef] [PubMed]

12. Rigano, D.; Aviello, G.; Bruno, M.; Formisano, C.; Rosselli, S.; Capasso, R.; Senatore, F.; Izzo, A.A.; Borrelli, F. Antispasmodic Effects and Structure-Activity Relationships of Labdane Diterpenoids from Marrubium globosum ssp. libanoticum. J. Nat. Prod. 2009, 72, 1477-1481. [CrossRef] [PubMed]

13. Hussain, A.; Perveen, S.; Malik, A.; Khan, A.N.; Tareen, R.B. Marrusidins A and B, New Epimeric Labdane Diterpenes from Marrubium anisodon. Helv. Chim. Acta 2010, 93, 1101-1104. [CrossRef]

14. Zaabat, N.; Hay, A.-E.; Michalet, S.; Darbour, N.; Bayet, C.; Skandrani, I.; Chekir-Ghedira, L.; Akkal, S.; Dijoux-Franca, M.-G. Antioxidant and Antigenotoxic Properties of Compounds Isolated from Marrubium deserti de Noé. Food Chem. Toxicol. 2011, 49, 3328-3335. [CrossRef] 
15. Zhang, J.-S.; Zou, Y.-H.; Zhao, J.-J.; Chen, Y.; Bao, J.-M.; Tang, G.-H. Three New Diterpenoids from Marrubium aschersonii. Phytochem. Lett. 2016, 16, 241-244. [CrossRef]

16. In addition to these named compounds, a large number of unnamed analogues have been isolated and characterized.

17. Zerbe, P.; Chiang, A.; Dullat, H.; O’Neil-Johnson, M.; Starks, C.; Hamberger, B.; Bohlmann, J. Diterpene synthases of the biosynthetic system of medicinally active diterpenoids in Marrubium vulgare. Plant J. 2014, 79, 914-927. [CrossRef]

18. Karunanithi, P.S.; Dhanota, P.; Addison, J.B.; Tong, S.; Fiehn, O.; Zerbe, P. Functional characterization of the cytochrome P450 monooxygenase CYP71AU87 indicates a role in marrubiin biosynthesis in the medicinal plant Marrubium vulgare. BMC Plant Biol. 2019, 19, 114. [CrossRef]

19. Mangoni, L.; Adinolfi, M.; Laonigro, G.; Caputo, R. Synthesis of Marrubiin. Tetrahedron 1972, 28, 611-621. [CrossRef]

20. Laonigro, G.; Lanzetta, R.; Parrilli, M.; Adinolfi, M.; Mangoni, L. The Configuration of the Diterpene Spiroethers from Marrubium vulgare and from Leonotis leonurus. Gazz. Chim. Ital. 1979, 109, 145-150.

21. Akahori, Y.; Yamakoshi, H.; Sawayama, Y.; Hashimoto, S.; Nakamura, S. Synthesis of Chiral Building Blocks for Oxygenated Terpenoids through a Simultaneous and Stereocontrolled Construction of Contiguous Quaternary Stereocenters by an Ireland-Claisen Rearrangement. J. Org. Chem. 2014, 79, 720-735. [CrossRef]

22. Saito, S.; Yamakoshi, H.; Nakamura, S. Second-Generation Synthesis of a Chiral Building Block for Oxygenated Terpenoids via a Ring-Contractive Coupling with a Secondary Alcohol. Heterocycles 2019, 99, 1086-1094.

23. Part of this work has already been communicated: Yamakoshi, H.; Sawayama, Y.; Akahori, Y.; Kato, M.; Nakamura, S. Total Syntheses of (+)-Marrubiin and (-)-Marrulibacetal. Org. Lett. 2016, 18, 3430-3433. [CrossRef] [PubMed]

24. For a review, see: Jeong, N. The Pauson-Khand Reaction. In Comprehensive Organic Synthesis II, 2nd ed.; Knochel, P., Molander, G.A., Eds.; Elsevier: Amsterdam, The Netherlands, 2014; Volume 5.24, pp. 1106-1178.

25. For a recent review on the use of Pauson-Khand reaction for synthesis of the natural products, see: Ma, K.; Martin, B.S.; Yin, X.; Dai, M. Natural Product Syntheses via Carbonylative Cyclizations. Nat. Prod. Rep. 2019, 36, 174-219. [CrossRef] [PubMed]

26. Yeh, M.-C.P.; Tsao, W.-C.; Ho, J.-S.; Tai, C.-C.; Chiou, D.-Y.; Tu, L.-H. Rhodium(I)-Catalyzed Intra molecular Cyclohexadienyl Pauson-Khand Reaction: Facile Approach to Tricarbocycles. Organometallics 2004, 23, 792-799. [CrossRef]

27. Sugihara, T.; Yamada, M.; Ban, H.; Yamaguchi, M.; Kaneko, C. Rate Enhancement of the Pauson-Khand Reaction by Primary Amines. Angew. Chem., Int. Ed. Engl. 1997, 36, 2801-2804. [CrossRef]

28. Yamanaka, M.; Nakamura, E. Density Functional Studies on the Pauson-Khand Reaction. J. Am. Chem. Soc. 2001, 123, 1703-1708. [CrossRef]

29. For a recent review, see: Lee, H.-W.; Kwong, F.-Y. A Decade of Advancements in Pauson-Khand-Type Reactions. Eur. J. Org. Chem. 2010, 789-811.

30. Krafft, M.E.; Boñaga, L.V.R.; Hirosawa, C. Practical Cobalt Carbonyl Catalysis in the Thermal Pauson-Khand Reaction: Efficiency Enhancement Using Lewis Bases. J. Org. Chem. 2001, 66, 3004-3020. [CrossRef]

31. Morimoto, T.; Fuji, K.; Tsutsumi, K.; Kakiuchi, K. CO-Transfer Carbonylation Reactions. A Catalytic Pauson-Khand-Type Reaction of Enynes with Aldehydes as a Source of Carbon Monoxide. J. Am. Chem. Soc. 2002, 124, 3806-3807. [CrossRef]

32. Yates, P.; Burke, P.M. Keto Ethers. IV. Products Formed on Reaction of Dihydro-2,2,5,5-tetramethyl3(2H)-furanone with Strong Acids. Can. J. Chem. 1987, 65, 1695-1704. [CrossRef]

33. The same results were obtained by the use of activated carbon supplied by Aldrich $\left(\mathrm{DARCO}{ }^{\circledR}\right)$, Tokyo Chemical Industry, and Taihei Chemical Industrial, suggesting that the reaction is not effected by contaminants.

34. Wheeler, D.M.S.; Wheeler, M.M.; Fetizon, M.; Castine, W.H. Synthesis of Diterpenoid Acids-VII: The Stereochemistry of Marrubiin. Tetrahedron 1967, 23, 3909-3921. [CrossRef]

35. When reductive workup with $\mathrm{NaBH}_{4}$ was employed after ozonolysis, enone $\mathbf{2 6}$ could be converted to lactone 23 in two steps without intervening purification, albeit in low yield (18\%).

36. Welch, S.C.; Rao, A.S.C.P.; Lyon, J.T.; Assercq, J.-M. Synthesis of 2,2-Disubstituted Oxetanes from Ketones with S-Methyl-S-(sodiomethyl)-N-(4-tolylsulfonyl)sulfoximine. J. Am. Chem. Soc. 1983, 105, 252-257. [CrossRef] 
37. Araki, S.; Butsugan, Y. Regio-controlled Prenylation and Geranylation of 3-Furylmethylmagnesium Bromide. Selective Syntheses of 3-Substituted Furanoid and 2-Substituted 3-Methylfuranoid Terpenes. Bull. Chem. Soc. Jpn. 1983, 56, 1446-1449. [CrossRef]

38. Cocker, W.; Cross, B.E.; Duff, S.R.; Edward, J.T.; Holley, T.F. The Constitution of Marrubiin. Part I. J. Chem. Soc. 1953, 2540-2548. [CrossRef]

39. Isomerization of Grignard reagent $\mathbf{3 2}$ was also observed by Sperry and Wright. Sperry, J.B.; Wright, D.L. Annulated Heterocycles through a Radical-Cation Cyclization: Synthetic and Mechanistic Studies. Tetrahedron 2006, 62, 6551-6557. [CrossRef]

40. Grignard reagent 34 was prepared from [2-(trimethylsilyl)furan-3-yl]methanol [52] by the following twostep sequence: 1) $\left.\mathrm{PBr}_{3}, \mathrm{Et}_{2} \mathrm{O}, 0{ }^{\circ} \mathrm{C}, 30 \mathrm{~min} ; 2\right) \mathrm{Mg}, \mathrm{Et}_{2} \mathrm{O},-10{ }^{\circ} \mathrm{C}, 1.5 \mathrm{~h}$.

41. Bures, E.J.; Keay, B.A. The Regiospecific Formation and Reactions of 4-Lithio-2-( $t$-butyldimethylsilyl)-3(hydroxymethyl)furan: An Approach to 3,4-Disubstituted Furans. Tetrahedron Lett. 1988, 29, 1247-1250. [CrossRef]

42. Harding, W.W.; Schmidt, M.; Tidgewell, K.; Kannan, P.; Holden, K.G.; Gilmour, B.; Navarro, H.; Rothman, R.B.; Prisinzano, T.E. Synthetic Studies of Neoclerodane Diterpenes from Salvia divinorum: Semisynthesis of Salvinicins A and B and Other Chemical Transformations of Salvinorin A. J. Nat. Prod. 2006, 69, 107-112. [CrossRef]

43. Nieto-Mendoza, E.; Guevara-Salazar, J.A.; Ramírez-Apan, M.T.; Frontana-Uribe, B.A.; Cogordan, J.A.; Cárdenas, J. Electro-Oxidation of Hispanolone and Anti-Inflammatory Properties of the Obtained Deriva tives. J. Org. Chem. 2005, 70, 4538-4541. [CrossRef]

44. Hönel, M.; Mosher, H.S. Selective Permanganate Oxidation of cis- vs. trans-2,5-Dihydro-2,5-dimethoxy furan. J. Org. Chem. 1985, 50, 4386-4388.

45. Kuwajima, I.; Urabe, H. Regioselective Synthesis of $\Delta^{3}$-Butenolides via Oxidation of 2-Trimethylsilyl furans. Tetrahedron Lett. 1981, 22, 5191-5194. [CrossRef]

46. Goldsmith, D.; Liotta, D.; Saindane, M.; Waykole, L.; Bowen, P. 3-Alkylfurans as Useful Synthetic Equiva lents for Substituted $\Delta^{2}$-Butenolides. Tetrahedron Lett. 1983, 24, 5835-5838. [CrossRef]

47. Shu, C.; Liu, M.-Q.; Sun, Y.-Z.; Ye, L.-W. Efficient Synthesis of $\gamma$-Lactones via Gold-Catalyzed Tandem Cycloisomerization/Oxidation. Org. Lett. 2012, 14, 4958-4961. [CrossRef] [PubMed]

48. Nemoto, H.; Fujita, S.; Nagai, M.; Fukumoto, K.; Kametani, T. A Novel Strategy for the Stereoselective Total Synthesis of C-17 Spiro Steroids. Total Synthesis of 19-Norcanrenone, a Formal Total Synthesis of 19-Norspironolactone. J. Am. Chem. Soc. 1988, 110, 2931-2938. [CrossRef]

49. Hansen, T.M.; Florence, G.J.; Lugo-Mas, P.; Chen, J.; Abrams, J.N.; Forsyth, C.J. Highly Chemoselective Oxidation of 1,5-Diols to $\delta$-Lactones with TEMPO/BAIB. Tetrahedron Lett. 2003, 44, 57-59. [CrossRef]

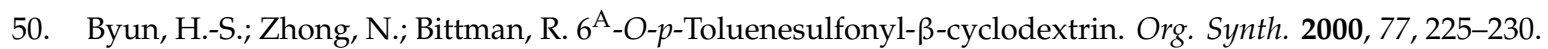

51. House, H.O.; Chu, C.-Y.; Wilkins, J.M.; Umen, M.J. The Chemistry of Carbanions. XXVII. A Convenient Precursor for the Generation of Lithium Organocuprates. J. Org. Chem. 1975, 40, 1460-1469. [CrossRef]

52. Dong, J.-Q.; Wong, H.N.C. Biomimetic Total Synthesis of ( \pm )-Pallavicinolide A. Angew. Chem., Int. Ed. 2009, 48, 2351-2354. [CrossRef]

53. Ahmed, A.; Hoegenauer, E.K.; Enev, V.S.; Hanbauer, M.; Kaehlig, H.; Öhler, E.; Mulzer, J. Total Synthesis of the Microtubule Stabilizing Antitumor Agent Laulimalide and Some Nonnatural Analogues: The Power of Sharpless' Asymmetric Epoxidation. J. Org. Chem. 2003, 68, 3026-3042. [CrossRef]

Sample Availability: Samples of the compounds are available from the authors. 